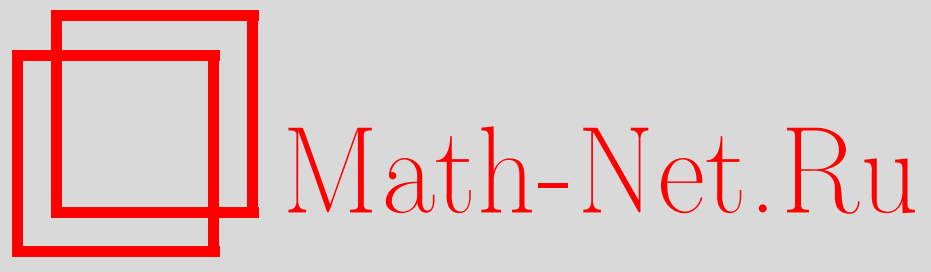

В. В. Зудилин, Арифметические гипергеометрические ряды, УМН, 2011, том 66, выпуск 2, 163-216

DOI: https://doi.org/10.4213/rm9420

Использование Общероссийского математического портала Math-Net.Ru подразумевает, что вы прочитали и согласны с пользовательским соглашением http://www . mathnet.ru/rus/agreement

Параметры загрузки:

IP: 54.224 .135 .184

26 апреля 2023 г., 13:48:26

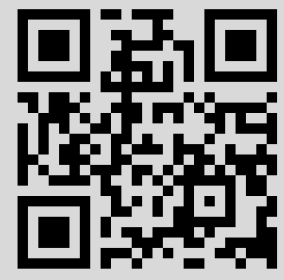




\title{
Арифметические гипергеометрические ряды
}

\author{
В. В. Зудилин
}

Основная задача данного обзора - дать общие характеристики вспомогательных гипергеометрических функций (и их обобщений), которые возникают в задачах теории чисел. Задуманные изначально как инструмент решения теоретико-числовых задач, гипергеометрические ряды в дальнейшем становятся связующим звеном между различными частями теории чисел и математики в целом.

Библиография: 183 названия.

Ключевые слова: гипергеометрический ряд, дзета-значение, диофантово приближение, мера иррациональности, модулярная форма, математика Рамануджана, дифференциальное уравнение Калаби-Яу, мера Малера, теория Уилфа-Цайльбергера, алгоритм созидательного телескопирования.

\section{СОДЕРЖАНИЕ}

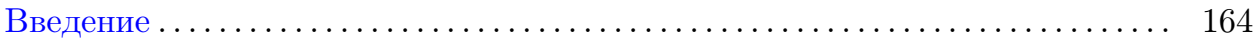

1. Арифметика значений дзета-функции Римана..................... 169

1.1. Теорема Апери .................................... 169

1.2. Гипергеометрические ряды и кратные интегралы . . . . . . . . . . 174

1.3. Совместные приближения к $\zeta(2)$ и $\zeta(3) \ldots \ldots \ldots \ldots \ldots \ldots \ldots \ldots$

1.4. q-Аналоги дзета-значений ........................... 181

1.5. Нижняя оценка для $\left\|(3 / 2)^{k}\right\|$ и проблема Варинга............. 184

2. Дифференциальные уравнения Калаби-Яу .................... 186

2.1. Арифметические дифференциальные уравнения второго и третьего порядка . . . . . . . . . . . . . . . . . . . 186

2.2. Арифметические дифференциальные уравнения четвертого и

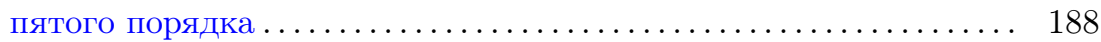

2.3. Семейство квинтик Калаби-Яу ........................ 190

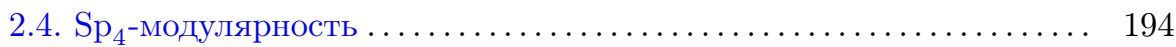

2.5. Формулы рамануджанова типа для $1 / \pi^{2} \ldots \ldots \ldots \ldots \ldots \ldots \ldots . \ldots \ldots$

Работа выполнена при поддержке Australian Research Council (проект DP110104419).

(C) В.В. Зудилин, 2011 
3. Суммы по решеткам и меры Малера............................ 200

3.1. $L$-ряды Дирихле и меры Малера.......................... 200

3.2. Четырехкратные суммы по решеткам.................... 203 Список литературы ...................................... 207

\section{Введение}

Арифметика, более известная в наши дни под именем теории чисел, есть душа математики, одна из ее самых старых частей. Карл Фридрих Гаусс, по мнению многих - величайший математик всех времен, называл математику "царицей наук", а теорию чисел - "царицей математики". Теория чисел известна наличием большого количества задач, понятных даже школьнику, но решаемых лишь с помощью глубоких методов современной математики. В этой связи стоит отметить последнюю теорему Ферма о неразрешимости уравнения $x^{n}+y^{n}=z^{n}$ в натуральных числах при $n>2$, которая, несмотря на ее название, в действительности не была доказана самим Ферма, а лишь совсем недавно Эндрю Вайлсом. Другой до сих пор не решенной задачей является гипотеза Римана о нулях функции

$$
\zeta(s):=\sum_{n=1}^{\infty} \frac{1}{n^{s}} .
$$

Широко признано, что ключевые проблемы теории чисел исключительно сложны и для решения требуют привлечения методов многих различных областей математики; развитие последних несомненно имеет исключительно большое значение и для математики в целом.

Одним из важнейших инструментов исследования задач теории чисел являются так называемые специальные функиии, происхождение и развитие которых на протяжении долгого времени диктовалось исключительно их полезностью в математической физике. Несмотря на то, что специальные функции обычно возникают в точно решаемых задачах физического происхождения [10], их значение для теории чисел нельзя переоценить. Все классические математические постоянные [55] такие, например, как $e, \pi$, значения логарифмов и обобщенных полилогарифмов, а также дзета-значения $\zeta(s)$ в целых точках $s>1$ являются специальными значениями некоторых специальных функций (не обязательно однозначно выбираемых).

Важный подкласс специальных функций составляют гипергеометрические функции [13], [61], [130], определяемые рядом

$$
{ }_{m} F_{m-1}\left(\begin{array}{cccc}
a_{1}, & a_{2}, & \ldots, & a_{m} \\
& b_{2}, & \ldots, & b_{m}
\end{array} \mid z\right)=\sum_{n=0}^{\infty} \frac{\left(a_{1}\right)_{n}\left(a_{2}\right)_{n} \cdots\left(a_{m}\right)_{n}}{\left(b_{2}\right)_{n} \cdots\left(b_{m}\right)_{n}} \frac{z^{n}}{n !}
$$

при $|z|<1$ и аналитически продолжимые на всю комплексную $\mathbb{C}$-плоскость с разрезом вдоль луча $[1,+\infty)$. Здесь

$$
(a)_{n}=\frac{\Gamma(a+n)}{\Gamma(a)}= \begin{cases}a(a+1) \cdots(a+n-1) & \text { при } n \geqslant 1, \\ 1 & \text { при } n=0\end{cases}
$$


обозначает символ Похгаммера (возрастающий факториал). Функция ${ }_{m} F_{m-1}$ в (1) удовлетворяет линейному дифференциальному уравнению порядка $m$

$$
\left(\theta \prod_{j=2}^{m}\left(\theta+b_{j}-1\right)-z \prod_{j=1}^{m}\left(\theta+a_{j}\right)\right) y=0, \quad \theta=z \frac{\mathrm{d}}{\mathrm{d} z},
$$

которое представляет собой частный случай дифференциального уравнения Пикара-Фукса [152]. Теория гипергеометрических функций дает возможность единого изложения многих достижений последних лет в теории чисел, включая, к примеру, загадочное доказательство Апери иррациональности $\zeta(3)$ [106] и быстро сходящиеся ряды Рамануджана для $\pi$ [30].

Неудивительным является то, что в действительности теория чисел задействует в своих целях лишь некоторый подкласс гипергеометрических функций - функций, отвечающих определенным арифметическим требованиям; к примеру, параметры ряда (1) должны быть рациональными или алгебраическими числами. Именно такие функции мы называем арифметическими гипергеометрическими рядами. Идея формализации указанных арифметических условий возникла на стыке теории струн в физике и алгебраической геометрии в математике. Так называемая "зеркальная симметрия" порождает естественную двойственность между некоторыми геометрическими объектами; по одну сторону этой двойственности находятся многообразия Калаби-Яу, периоды которых удовлетворяют специальным дифференциальным уравнениям Пикара-Фукса. Заключенная в уравнениях арифметическая информация указывает (как ожидается) путь к реконструкции двойственных объектов. Совместная работа [6] Г. Алмквиста и автора представляет собой одну из первых попыток продвижения теории дифференциальных уравнений Калаби-Яу на рынок теории чисел. Важным для современной математики является дальнейшее развитие данной тематики в связи с задачами теории чисел [154], [181], алгебраической геометрии [4], математической физики [73] и других областей.

Скромная цель данного обзора - продемонстрировать, как арифметические гипергеометрические ряды связывают некоторые внешне различные объекты исследований, а также объяснить лежащий в основе этой взаимосвязи арифметический и аналитический аппараты. Более конкретно, мы рассматриваем следующие вопросы:

(1) арифметические свойства значений дзета-функции Римана $\zeta(s)$ и ее обобщений в целых точках $s>1$;

(2) значимость в арифметике дифференциальных уравнений Калаби-Яу и обобщенных рядов рамануджанова типа для представления $\pi$;

(3) значения мер Малера в терминах гипергеометрических и специальных функций.

В §1 мы обсуждаем арифметические свойства значений дзета-функции Римана. Арифметическая природа значений $\zeta(s)$ в целых $s=2,3,4, \ldots$ (дзетазначений) хорошо изучена для четных $s$ благодаря полученным Эйлером представлениям для $\zeta(2 k)$, влекущим $\zeta(2 k) / \pi^{2 k} \in \mathbb{Q}$, и доказательству Линдемана (1882 г.) трансцендентности числа $\pi$. Для нечетных $s$ имеются лишь результаты об иррациональности (но не трансцендентности, хотя все такие дзета-значения, как ожидается, трансцендентны). После публикации Апери в 1978 г. 
неожиданного доказательства иррациональности $\zeta(3)$, следует упомянуть совсем недавние результаты К. Болла и Т. Ривоаля [15], а также результаты автора [163], [173], в которых помимо прочего показано, что последовательность $\zeta(3), \zeta(5), \zeta(7), \ldots$ содержит бесконечное количество иррациональных чисел. Более того, известно, что среди чисел

$$
\zeta(s+2), \zeta(s+4), \ldots, \zeta(8 s-3), \zeta(8 s-1),
$$

где $s>1$ нечетно, содержится по крайней мере одно иррациональное число и что по крайней мере одно из чисел $\zeta(5), \zeta(7), \zeta(9), \zeta(11)$ иррационально. Задействованный в доказательствах гипергеометрический аппарат позволяет получать аналогичные результаты для значений других рядов Дирихле [119], для $q$-аналогов дзета-значений [84], [172] и, более обще, для значений многих других классических q-рядов [37], [39], [97].

В 22 дается формальное определение дифференциальных уравнений Калаби-Яу и обзор их свойств монодромии. Огромная база данных по таким уравнениям приведена в табличной форме в [3]. При этом одним из вопросов данной тематики является выяснение взаимосвязей между различными примерами таких специальных уравнений и их решений - по сути арифметическими гипергеометрическими рядами; эти взаимосвязи представляют собой алгебраические преобразования. Приведем пример такого преобразования из недавней работы [5]. Определим два двойных гипергеометрических ряда

$$
F(z)=\sum_{n=0}^{\infty} z^{n}\left(\sum_{k=0}^{n}(-1)^{k}\left(\begin{array}{l}
n \\
k
\end{array}\right) \frac{\left(\frac{1}{2}\right)_{k}^{2}}{k !^{2}}\right)^{2}
$$

и

$$
\widehat{F}(z)=\sum_{n=0}^{\infty} z^{n} \frac{\left(\frac{1}{4}\right)_{n}\left(\frac{3}{4}\right)_{n}}{n !^{2}} \sum_{k=0}^{n}(-1)^{k}\left(\begin{array}{l}
n \\
k
\end{array}\right) \frac{\left(\frac{1}{4}\right)_{k}\left(\frac{3}{4}\right)_{k}}{k !^{2}}
$$

Тогда

$$
F(z)=\frac{1}{\sqrt{1-6 z+z^{2}}} \widehat{F}\left(\frac{-16 z(1-z)^{2}}{\left(1-6 z+z^{2}\right)^{2}}\right) .
$$

В $\S 2$ также рассматриваются обобщенные формулы Рамануджана для $\pi$. В настоящее время доказательства классических формул Рамануджана для $1 / \pi$

$$
\begin{gathered}
\sum_{n=0}^{\infty} \frac{\left(\frac{1}{2}\right)_{n}^{3}}{n !^{3}}(6 n+1) \frac{1}{4^{n}}=\frac{4}{\pi}, \\
\sum_{n=0}^{\infty} \frac{\left(\frac{1}{2}\right)_{n}\left(\frac{1}{6}\right)_{n}\left(\frac{5}{6}\right)_{n}}{n !^{3}}(5418 n+263) \frac{(-1)^{n}}{80^{3 n}}=\frac{640 \sqrt{15}}{3 \pi}
\end{gathered}
$$

(см., например, [111]) могут быть получены единообразно благодаря доступному модулярному и гипергеометрическому аппаратам [30], [16], [181]. Подобная формула

$$
\sum_{n=0}^{\infty} \frac{\left(\frac{1}{6}\right)_{n}\left(\frac{1}{2}\right)_{n}\left(\frac{5}{6}\right)_{n}}{n !^{3}}(545140134 n+13591409) \cdot \frac{(-1)^{n}}{53360^{3 n+2}}=\frac{3}{2 \pi \sqrt{10005}},
$$


установленная Чудновскими [43], использовалась Беллардом в декабре 2009 г. для вычисления 2.7 триллионов десятичных знаков числа $\pi$ (на одной рабочей станции). Такие ряды приведены Рамануджаном в 1914 г., однако первые полные доказательства этих представлений получены лишь в 1980-х гг. в [30]. Обобщения этих формул (на случай пяти символов Похгаммера в числителе и $n !^{5}$ в знаменателе) были обнаружены лишь недавно Х. Гиллерой [65]-[68], а также Г. Алмквистом и Х. Гиллерой [2]. Известно одиннадцать представлений последнего типа, из которых строго доказаны лишь четыре (с использованием гипергеометрических алгоритмов), в то время как остальные семь такие, как

$$
\sum_{n=0}^{\infty} \frac{\left(\frac{1}{2}\right)_{n}\left(\frac{1}{3}\right)_{n}\left(\frac{2}{3}\right)_{n}\left(\frac{1}{6}\right)_{n}\left(\frac{5}{6}\right)_{n}}{n !^{5}}\left(5418 n^{2}+693 n+29\right) \frac{(-1)^{n}}{80^{3 n}} \stackrel{?}{=} \frac{128 \sqrt{5}}{\pi^{2}}
$$

и

$$
\sum_{n=0}^{\infty} \frac{(6 n) !}{n ! 6}\left(532 n^{2}+126 n+9\right) \frac{1}{10^{6 n}} \stackrel{?}{=} \frac{375}{4 \pi^{2}},
$$

не поддаются исследованию имеющимися методами. В этом направлении важно понять структуру подобных формул и развить технику их доказательства. Соответствующие гипергеометрические дифференциальные уравнения представляют собой частные случаи дифференциальных уравнений Калаби-Яу.

В § 3 мы рассматриваем меры Малера. Для многочлена Лорана

$$
P\left(x_{1}, \ldots, x_{n}\right) \in \mathbb{C}\left[x_{1}^{ \pm 1}, \ldots, x_{n}^{ \pm 1}\right]
$$

(логарифмическая) мера Малера определяется как

$$
\mathrm{m}(P):=\int \cdots \int_{[0,1]^{n}} \log \left|P\left(e^{2 \pi i \theta_{1}}, \ldots, e^{2 \pi i \theta_{n}}\right)\right| \mathrm{d} \theta_{1} \cdots \mathrm{d} \theta_{n} .
$$

Эта характеристика была впервые введена Эрмитом 150 лет назад и подробно исследовалась Малером в 1960-х гг. Мера Малера необходима для понимания точной структуры нулей целочисленных многочленов и, как следствие, трансцендентности. В 1998 г. Д. Бойд [34] на основе огромного объема вычислений выдвинул ряд поразительных и неожиданных гипотез о соотношениях между $L$-рядами Дирихле (обобщенными дзета-значениями) для эллиптических кривых и мерами Малера многочленов, отвечающих многообразиям нулей соответствующих кривых.

Первый результат в этом направлении был получен К. Денингером [47]. Опираясь на гипотезы Бейлинсона, он показал, что

$$
\mathrm{m}\left(1+x+x^{-1}+y+y^{-1}\right)=r L^{\prime}(E, 0)=r \frac{15}{4 \pi^{2}} L(E, 2)
$$

с точностью до рационального множителя $r$, где $E$ - эллиптическая кривая с кондуктором 15. На основании численных расчетов [34] множитель $r$ предполагается равным 1. В соответствии с теоремой о модулярности эллиптических 
кривых ожидаемое равенство можно записать в следующем виде:

$$
\begin{aligned}
\mathrm{m} & \left(1+x+x^{-1}+y+y^{-1}\right) \\
& =\frac{540}{\pi^{2}} \sum_{\substack{n_{i}=-\infty \\
i=1,2,3,4}}^{\infty} \frac{(-1)^{n_{1}+n_{2}+n_{3}+n_{4}}}{\left(\left(6 n_{1}+1\right)^{2}+3\left(6 n_{2}+1\right)^{2}+5\left(6 n_{3}+1\right)^{2}+15\left(6 n_{4}+1\right)^{2}\right)^{2}} .
\end{aligned}
$$

$K$-теория рассматривается как естественный аппарат, с помощью которого гипотезы Бойда могут быть доказаны [120], [121], [36], [98]. Тем не менее наибольший прогресс в этом направлении достигнут комбинированием $K$-теоретического подхода с методами теории модулярных функций. Такая техника получила развитие в работах Ф. Родригеса-Виллегаса, и позднее в работах М. Ж. Бертен, Д. Бойда и других [20], [35], [120]. На самом деле, требование модулярности является очень ограничительным. Большинство гипотез Бойда можно перефразировать в терминах тождеств для гипергеометрических рядов. Такой прием был использован М. Лалан и М. Д. Роджерсом [87], [123] для вывода ряда новых тождеств для мер Малера. К примеру, используя гипергеометрическое равенство [123]

$$
\mathrm{m}\left(1+x+x^{-1}+y+y^{-1}\right)=4 \sum_{n=0}^{\infty}\left(\begin{array}{c}
2 n \\
n
\end{array}\right)^{2} \frac{(1 / 16)^{2 n+1}}{2 n+1},
$$

доказательство представления (10) можно свести к проверке того, что четырехкратная сумма дает значение гипергеометрического ряда в правой части (11). Использование гипергеометрического аппарата играет центральную роль при получении в [125] доказательства равенства (10), а также при доказательстве некоторых других гипотез из списка Бойда в [124], которые оставались открытыми в течение многих лет.

Из (10) и (11) следует, что

$$
\begin{aligned}
& \frac{540}{\pi^{2}} \sum_{\substack{n_{i}=-\infty \\
i=1,2,3,4}}^{\infty} \frac{(-1)^{n_{1}+n_{2}+n_{3}+n_{4}}}{\left(\left(6 n_{1}+1\right)^{2}+3\left(6 n_{2}+1\right)^{2}+5\left(6 n_{3}+1\right)^{2}+15\left(6 n_{4}+1\right)^{2}\right)^{2}} \\
& \quad=4 \sum_{n=0}^{\infty}\left(\begin{array}{c}
2 n \\
n
\end{array}\right)^{2} \frac{(1 / 16)^{2 n+1}}{2 n+1} .
\end{aligned}
$$

Это тождество - тонкий аналитический результат, связывающий сложную сумму по решетке с простой гипергеометрической функцией ${ }_{3} F_{2}$. Суммы по решеткам интенсивно изучались в физике, где они часто возникают при вычислении электростатических потенциалов кристаллических решеток (см., например, [30], [59], [63] и [159]). В этой связи представляется неслучайным, что равенство (12) поразительно напоминает известную формулу Рамануджана [55] для постоянной Каталана:

$$
\frac{1}{\pi} \sum_{n=0}^{\infty} \frac{(-1)^{n}}{(2 n+1)^{2}}=\sum_{n=0}^{\infty}\left(\begin{array}{c}
2 n \\
n
\end{array}\right)^{2} \frac{(1 / 4)^{2 n+1}}{2 n+1} .
$$

Отметим, что постоянная Каталана является одной из простейших арифметических величин, иррациональность которой до сих пор не доказана. Тот факт, 
что правые части равенств (12) и (13) являются арифметическими значениями одной и той же гипергеометрической функции, дает основания полагать, что большое количество тождеств с мерой Малера могут быть доказаны с использованием арифметической гипергеометрической техники. Сказанное прекрасно иллюстрирует необходимость лучшего понимания таких подчас строгих, а иногда экспериментальных вычислений.

На протяжении многих лет Анатолий Алексеевич Карацуба проявлял интерес к моим арифметико-гипергеометрическим исследованиям. Его поддержка носила не только моральный характер, поскольку в 2006-2008 гг. я работал под его руководством в отделе теории чисел Математического института им. В. А. Стеклова. Его внезапный уход явился невосполнимой потерей не только для теории чисел и математики вообще, но и стал для меня потерей учителя и друга. Данный обзор является данью памяти Анатолия Алексеевича.

Мое интеллектуальное удовольствие от работы с арифметическими гипергеометрическими рядами и мои знания в этой области были бы невозможны без моих коллег, соавторов и друзей. Пользуясь случаем, я хотел бы выразить глубокую признательность Г. Алмквисту, А. И. Аптекареву, Д. Бертрану, Дж. Борвайну, П. Бундшу, Х. Х. Чану, В. В. Голышеву, С. Фишлеру, Х. Гиллере, М. Уттнеру, К. Краттенталеру, Л. Лонг, Т. Матало-ахо, А. Меллиту, Ю. В. Нестеренко, Я. Оно, Дж. Рину, Т. Ривоалю, И. П. Рочеву, М. Роджерсу, В. Н. Сорокину, В.П. Спиридонову, А. Штраубу, К. Ваананену, В. Ван Ассе, Д. ван Стратену, К. Виоле, М. Вальдшмидту, Дж. Вану, О. Варнаару, Й. Янгу, Д. Загиру и Д. Цайльбергеру.

\section{1. Арифметика значений дзета-функции Римана}

1.1. Теорема Апери. Изучение сумм вида

$$
\zeta(s)=\sum_{n=1}^{\infty} \frac{1}{n^{s}}
$$

при целых положительных значениях параметра $s$ восходит к Л. Эйлеру [53], [54]. В частности, им доказана расходимость ряда в (14) при $s=1$, его сходимость при $s>1$, а также знаменитые соотношения

$$
2 \sum_{n=1}^{\infty} \frac{1}{n^{2 k}}=-\frac{(2 \pi i)^{2 k} B_{2 k}}{(2 k) !} \quad \text { для } \quad k=1,2,3, \ldots,
$$

связывающие значения ряда при четных положительных $s$ с архимедовой постоянной $\pi=3.14159265 \ldots$ (см. [55; 1.4$])$ и числами Бернулли $B_{s} \in \mathbb{Q}$; последние могут быть определены с помощью производящей функции

$$
\frac{z}{e^{z}-1}=1-\frac{z}{2}+\sum_{s=2}^{\infty} B_{s} \frac{z^{s}}{s !}=1-\frac{z}{2}+\sum_{k=1}^{\infty} B_{2 k} \frac{z^{2 k}}{(2 k) !} .
$$

В 1882 году Ф. Линдеман [90] доказал трансцендентность числа $\pi$ и, тем самым, трансцендентность $\zeta(s)$ для четных $s$. 
Лишь век спустя после Эйлера Б. Риман [115] рассмотрел ряд в (14) как функцию комплексного переменного $s$. Этот ряд представляет в области $\operatorname{Re} s>1$ аналитическую функцию, которая может быть продолжена на всю комплексную плоскость до мероморфной функции $\zeta(s)$. Именно это аналитическое продолжение и ряд важных свойств функции $\zeta(s)$ были открыты Риманом в его мемуаре о простых числах. Дзета-функция Римана и ее обобщения играют фундаментальную роль в аналитической теории чисел [145]. Далее мы обсуждаем лишь арифметические и аналитические свойства значений эйлеровых сумм $\zeta(s)$ в (14) при целых положительных $s>1$ и обобщений этих чисел. Для краткости мы будем называть величины (14) при целых положительных $s$ дзета-значениями, а также четными и нечетными дзета-значениями в зависимости от четности $s$.

Как было отмечено выше, трансцендентность (а значит, и иррациональность) четных дзета-значений следует из классических результатов Эйлера и Линдемана. Формулы, подобные (15), для нечетных дзета-значений неизвестны, и предположительно $\zeta(2 k+1) / \pi^{2 k+1}$ не является рациональным числом ни для какого целого $k \geqslant 1$. Арифметическая природа нечетных дзета-значений казалась неприступной вплоть до 1978 года, когда Р. Апери [12] предъявил последовательность рациональных приближений, доказывающих иррациональность числа $\zeta(3)$.

История этого открытия так же, как и строгое математическое обоснование наблюдений Апери, изложены в [106]. Число Ц(3) известно в наши дни как постоянная Апери (см., например, [55; §1.6]). В качестве рациональных приближений к $\zeta(3)$ Апери выбирает последовательность $v_{n} / u_{n} \in \mathbb{Q}, n=0,1,2, \ldots$, где знаменатели $\left\{u_{n}\right\}=\left\{u_{n}\right\}_{n=0,1, \ldots}$ и числители $\left\{v_{n}\right\}=\left\{v_{n}\right\}_{n=0,1, \ldots}$ Удовлетворяют одной и той же полиномиальной рекурсии

$$
(n+1)^{3} u_{n+1}-(2 n+1)\left(17 n^{2}+17 n+5\right) u_{n}+n^{3} u_{n-1}=0
$$

с начальными данными

$$
u_{0}=1, \quad u_{1}=5, \quad v_{0}=0, \quad v_{1}=6 .
$$

Тогда

$$
\lim _{n \rightarrow \infty} \frac{v_{n}}{u_{n}}=\zeta(3) .
$$

При этом не менее важным обстоятельством являются неожиданные (с точки зрения рекурсии (16)) включения

$$
u_{n}=\sum_{k=0}^{n}\left(\begin{array}{l}
n \\
k
\end{array}\right)^{2}\left(\begin{array}{c}
n+k \\
k
\end{array}\right)^{2} \in \mathbb{Z}, \quad D_{n}^{3} v_{n} \in \mathbb{Z}, \quad n=0,1,2, \ldots,
$$

где через $D_{n}$ обозначено наименьшее общее кратное чисел $1,2, \ldots, n$ (и $D_{0}=1$ для полноты). Применение теоремы Пуанкаре (см., например, [62]) к разностному уравнению (16) приводит согласно (18) к предельным соотношениям

$$
\begin{gathered}
\lim _{n \rightarrow \infty}\left|u_{n} \zeta(3)-v_{n}\right|^{1 / n}=(\sqrt{2}-1)^{4}, \\
\lim _{n \rightarrow \infty}\left|u_{n}\right|^{1 / n}=\lim _{n \rightarrow \infty}\left|v_{n}\right|^{1 / n}=(\sqrt{2}+1)^{4},
\end{gathered}
$$


где числа $(\sqrt{2}-1)^{4}$ и $(\sqrt{2}+1)^{4}$ являются корнями характеристического многочлена $\lambda^{2}-34 \lambda+1$ рекурсии (16). Собранная информация о свойствах последовательностей $\left\{u_{n}\right\}$ и $\left\{v_{n}\right\}$ доказывает, что число $\zeta(3)$ не может быть рациональным. Действительно, в предположении $\zeta(3)=a / b$, где $a, b \in \mathbb{Z}$, линейные формы $r_{n}=b D_{n}^{3}\left(u_{n} \zeta(3)-v_{n}\right)$ являются целыми числами, ненулевыми ввиду $(20)$. С другой стороны, $D_{n}^{1 / n} \rightarrow e$ при $n \rightarrow \infty$ согласно асимптотическому закону распределения простых чисел (см., например, [145; гл. II, §3]); следовательно,

$$
\lim _{n \rightarrow \infty}\left|r_{n}\right|^{1 / n}=e^{3}(\sqrt{2}-1)^{4}=0.59126300 \ldots<1,
$$

что при достаточно большом $n$ вступает в противоречие с оценкой $\left|r_{n}\right| \geqslant 1$ для целых ненулевых $r_{n}$. Более того, дополнительные предельные соотношения (21) и стандартные соображения (см., например, [75; лемма 3.1]) позволяют измерить иррациональность постоянной Апери количественно:

$$
\mu(\zeta(3)) \leqslant 1+\frac{4 \log (\sqrt{2}+1)+3}{4 \log (\sqrt{2}+1)-3}=13.41782023 \ldots .
$$

Здесь и далее показателем иррациональности $\mu(\alpha)$ вещественного иррационального числа $\alpha$ называется величина

$$
\begin{aligned}
& \mu=\mu(\alpha)=\inf \{c \in \mathbb{R}: \text { неравенство }|\alpha-a / b| \leqslant|b|^{-c} \text { имеет } \\
&\text { конечное число решений в } a, b \in \mathbb{Z}\} ;
\end{aligned}
$$

в случае $\mu(\alpha)<+\infty$ говорят, что $\alpha$ - нелиувиллево число.

Оригинальные рассуждения Апери (именно, соотношения (16)-(21)) были настолько загадочны, что интерес к теореме Апери не ослабевает и в наши дни. Феномен последовательности рациональных приближений Апери неоднократно переосмысливался с точки зрения различных методов (см. [21], [24], [57], [72], [75], [101], [108], [113], [132], [134], [135], [144], [154], [155], [160], [182]). Новые подходы позволили усилить результат Апери количественно - получить лучшую оценку для показателя иррациональности числа Ц(3) (последние этапы соревнования в этом направлении - работы [76], [113]). Мы прежде всего отметим явные формулы для последовательности $u_{n} \zeta(3)-v_{n}$, которые играют роль в дальнейшем изложении: представление Бэйкерса [21]

$$
u_{n} \zeta(3)-v_{n}=\iiint_{[0,1]^{3}} \frac{x^{n}(1-x)^{n} y^{n}(1-y)^{n} z^{n}(1-z)^{n}}{(1-(1-x y) z)^{n+1}} \mathrm{~d} x \mathrm{~d} y \mathrm{~d} z
$$

в виде кратного вещественного интеграла, ряд Гутника-Нестеренко [72], [101]

$$
u_{n} \zeta(3)-v_{n}=-\left.\frac{1}{2} \sum_{\nu=1}^{\infty} \frac{\mathrm{d}}{\mathrm{d} t}\left(\frac{(t-1)(t-2) \cdots(t-n)}{t(t+1)(t+2) \cdots(t+n)}\right)^{2}\right|_{t=\nu},
$$

а также ряд Болла [15]

$$
u_{n} \zeta(3)-v_{n}=\left.n !^{2} \sum_{\nu=1}^{\infty}\left(t+\frac{n}{2}\right) \frac{(t-1) \cdots(t-n) \cdot(t+n+1) \cdots(t+2 n)}{t^{4}(t+1)^{4} \cdots(t+n)^{4}}\right|_{t=\nu} .
$$


Отметим, что с помощью своего метода "ускорения сходимости" Апери [12], [106] установил также иррациональность числа $\zeta(2)$ без явного применения формулы $\zeta(2)=\pi^{2} / 6$. На этот раз, знаменатели $\left\{u_{n}^{\prime}\right\}$ и числители $\left\{v_{n}^{\prime}\right\}$ линейных приближающих форм $u_{n}^{\prime} \zeta(2)-v_{n}^{\prime}, n=0,1,2, \ldots$, удовлетворяют рекурсии

$$
(n+1)^{2} u_{n+1}-\left(11 n^{2}+11 n+3\right) u_{n}-n^{2} u_{n-1}=0
$$

с начальными данными

$$
u_{0}^{\prime}=1, \quad u_{1}^{\prime}=3, \quad v_{0}^{\prime}=0, \quad v_{1}^{\prime}=5 ;
$$

при этом

$$
u_{n}^{\prime}=\sum_{k=0}^{n}\left(\begin{array}{l}
n \\
k
\end{array}\right)^{2}\left(\begin{array}{c}
n+k \\
k
\end{array}\right) \in \mathbb{Z}, \quad D_{n}^{2} v_{n}^{\prime} \in \mathbb{Z}, \quad n=0,1,2, \ldots,
$$

и

$$
\begin{aligned}
& \lim _{n \rightarrow \infty}\left|u_{n}^{\prime} \zeta(2)-v_{n}^{\prime}\right|^{1 / n}=\left(\frac{\sqrt{5}-1}{2}\right)^{5}<e^{-2}, \\
& \lim _{n \rightarrow \infty}\left|u_{n}\right|^{1 / n}=\lim _{n \rightarrow \infty}\left|v_{n}\right|^{1 / n}=\left(\frac{\sqrt{5}+1}{2}\right)^{5} .
\end{aligned}
$$

Данная последовательность приближений приводит также к оценке

$$
\mu(\zeta(2))=\mu\left(\pi^{2}\right) \leqslant 1+\frac{5 \log ((\sqrt{5}+1) / 2)+2}{5 \log ((\sqrt{5}+1) / 2)-2}=11.85078219 \ldots
$$

для показателя иррациональности числа $\pi^{2}$. Приближения Апери к $\zeta(2)$ могут быть представлены в виде двукратного вещественного интеграла [21]

$$
u_{n}^{\prime} \zeta(2)-v_{n}^{\prime}=(-1)^{n} \iint_{[0,1]^{2}} \frac{x^{n}(1-x)^{n} y^{n}(1-y)^{n}}{(1-x y)^{n+1}} \mathrm{~d} x \mathrm{~d} y,
$$

а также в виде гипергеометрического ряда

$$
u_{n}^{\prime} \zeta(2)-v_{n}^{\prime}=\left.(-1)^{n} \sum_{\nu=1}^{\infty} \frac{n ! \cdot(t-1)(t-2) \cdots(t-n)}{t^{2}(t+1)^{2}(t+2)^{2} \cdots(t+n)^{2}}\right|_{t=\nu} .
$$

Теорема Апери является первым существенным продвижением в решении следующей задачи (которую по праву можно назвать фольклорной; печатное упоминание см., например, в [129; заключительные замечания]): доказать иррачиональность чисел $\zeta(2 k+1)$ для $k=1,2,3, \ldots$.

K сожалению, естественные обобщения конструкции Апери приводят к линейным формам, содержащим значения дзета-функции как в нечетных, так и в четных точках; это обстоятельство не позволяло получить результаты об иррациональности $\zeta(s)$ для нечетных $s \geqslant 5$. Лишь в 2000 году Т. Ривоаль [116], используя обобщение представления Болла (24), построил линейные формы, содержащие только нечетные дзета-значения и позволяющие доказать следующий результат. 
Teоpema 1. Cреди чисел

$$
\zeta(3), \zeta(5), \zeta(7), \zeta(9), \zeta(11), \ldots
$$

имеется бесконечно много иррациональных. Более точно, для размерности $\delta(s)$ пространств, порожденных над $\mathbb{Q}$ числами $1, \zeta(3), \zeta(5), \ldots, \zeta(s-2), \zeta(s)$, где s нечетно, справедлива оченка

$$
\delta(s) \geqslant \frac{\log s}{1+\log 2}(1+o(1)) \quad \text { npu } s \rightarrow \infty .
$$

Линейные приближающие формы Ривоаля в [116] записываются в виде

$$
F_{n}=F_{s, r, n}=\left.n !^{s+1-2 r} \sum_{\nu=1}^{\infty}\left(t+\frac{n}{2}\right) \frac{\prod_{j=1}^{r n}(t-j) \cdot \prod_{j=1}^{r n}(t+n+j)}{\prod_{j=0}^{n}(t+j)^{s+1}}\right|_{t=\nu},
$$

$s$ нечетно,

где вспомогательный параметр $r<s / 2$ имеет порядок $r \sim s / \log ^{2} s$; в частности, ряд $F_{3,1, n}$ совпадает с представлением (24) для последовательности Апери. Раскладывая рациональную функцию параметра $t$ под зна́ком суммирования в сумму простейших дробей и используя идеи работ [101] и [103], можно показать, что справедливы включения

$$
2 D_{n}^{s+1} F_{n} \in \mathbb{Z} \zeta(s)+\mathbb{Z} \zeta(s-2)+\cdots+\mathbb{Z} \zeta(5)+\mathbb{Z} \zeta(3)+\mathbb{Z} .
$$

Кроме того, явные формулы (32) для линейных форм от нечетных дзета-значений позволяют вычислить асимптотическое поведение этих форм и их коэффициентов при $n \rightarrow \infty$. Заключительный этап доказательства теоремы Ривоаля применение критерия линейной независимости Нестеренко [100].

Тот факт, что величины (32) являются $\mathbb{Q}$-линейными формами от 1 и дзетазначений одной четности, связан со специальной симметрией рациональной функции параметра $t$, стоящей в (32) под знаком суммы. Возможность использования менее экзотической рациональной функции обсуждается в работах [72], [77], [117]: в итоге получаются результаты о размерности пространств, порожденных над $\mathbb{Q}$ значениями полилогарифмов

$$
\operatorname{Li}_{s}(z)=\sum_{n=1}^{\infty} \frac{z^{n}}{n^{s}}
$$

в рациональной точке $z, 0<|z| \leqslant 1$.

Несмотря на то что доказательство теоремы Ривоаля использует некоторое обобщение конструкции для доказательства теоремы Апери, ее результат дает лишь частичное решение задачи об иррациональности нечетных дзета-значе-

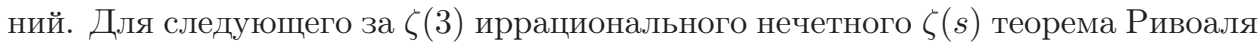
устанавливает лишь диапазон [15]: $5 \leqslant s \leqslant 169$. Дифференцирование рациональной функции под зна́ком суммирования (подобно представлению (23)) дает возможность строить $\mathbb{Q}$-линейные формы от нечетных дзета-значений, не содержащие $\zeta(3)$. Это позволяет доказать [163], [118], что по крайней мере одно из 
девяти нечетных дзета-значений $\zeta(5), \zeta(7), \ldots, \zeta(21)$ иррационально. Наконец, использование наиболее общей формы конструкции, предложенной в работах Ривоаля, а также арифметического метода (см., например, [42], [126], [75]), традиционно применяемого для улучшения оценок меры иррациональности чисел, позволяет получить [162], [173] следующий результат.

ТЕОРема 2. Одно из чисел

$$
\zeta(5), \zeta(7), \zeta(9), \zeta(11)
$$

иррационально.

Отметим, что использованная техника успешно работает и в других арифметических задачах: в [119] аналоги теоремы 1 и 2 установлены для значений бета-функции Дирихле

$$
\beta(s)=\sum_{n=0}^{\infty} \frac{(-1)^{n}}{(2 n+1)^{s}}
$$

в четных точках $s \geqslant 2$. В совместной работе [58] приводится некоторое усиление (и дается новое доказательство) критерия линейной независимости Нестеренко [100], с помощью которого улучшаются диапазоны изменения аргумента дзета-функции из [15] и [163].

1.2. Гипергеометрические ряды и кратные интегралы. Доказательство Бэйкерса [21] иррациональности $\zeta(2)$ и $\zeta(3)$, использующее интегральные представления (30) и (22), простое и короткое. Именно это послужило серьезным основанием для дальнейшего применения кратных интегралов с целью количественного усиления и обобщения результатов Апери (см. [50], [75], [76], [112], [113], [140], [141], [142], [144]). О. Василенко [140] предложил рассматривать следующее семейство $s$-кратных интегралов, обобщающих интегралы Бэйкерса:

$$
J_{s, n}=\int_{[0,1]^{s}} \ldots \int \frac{\prod_{j=1}^{s} x_{j}^{n}\left(1-x_{j}\right)^{n}}{Q_{s}\left(x_{1}, \ldots, x_{s}\right)^{n+1}} \mathrm{~d} x_{1} \cdots \mathrm{d} x_{s}
$$

где

$$
Q_{s}\left(x_{1}, \ldots, x_{s}\right)=1-x_{1}\left(1-x_{2}\left(1-\cdots\left(1-x_{s-1}\left(1-x_{s}\right)\right) \cdots\right)\right) .
$$

Первым продвижением в этом направлении стала работа Д. Васильева [142], в которой он изучил интегралы $J_{4, n}, J_{5, n}$ и доказал, что

$$
4 D_{n}^{4} J_{4, n} \in \mathbb{Z} \zeta(4)+\mathbb{Z} \zeta(2)+\mathbb{Z}, \quad D_{n}^{5} J_{5, n} \in \mathbb{Z} \zeta(5)+\mathbb{Z} \zeta(3)+\mathbb{Z},
$$

а также что линейные формы в (33) стремятся (достаточно быстро) к нулю при $n \rightarrow \infty$ (к сожалению, не настолько быстро, чтобы получить новые результаты об иррациональности дзета-значений). Включения $D_{n}^{2} J_{2, n} \in \mathbb{Z} \zeta(2)+\mathbb{Z}$, $D_{n}^{3} J_{3, n} \in \mathbb{Z} \zeta(3)+\mathbb{Z}$, доказанные Бэйкерсом в [21], и (35) дали Васильеву основание высказать следующее предположение:

$$
\begin{gathered}
2^{s-2} D_{n}^{s} J_{s, n} \in \mathbb{Z} \zeta(s)+\mathbb{Z} \zeta(s-2)+\cdots+\mathbb{Z} \zeta(4)+\mathbb{Z} \zeta(2)+\mathbb{Z} \quad \text { для } s \text { четного, } \\
D_{n}^{s} J_{s, n} \in \mathbb{Z} \zeta(s)+\mathbb{Z} \zeta(s-2)+\cdots+\mathbb{Z} \zeta(5)+\mathbb{Z} \zeta(3)+\mathbb{Z} \quad \text { для } s \text { нечетного. }
\end{gathered}
$$


Несмотря на справедливость этого предположения в случае $s=2,3,4,5$, уверенность автора [142] в том, что (36) верно для всех $s$, разделяли немногие. Причиной последнего является другая неверная гипотеза, именно $2^{s-2} D_{n}^{s} J_{s, n} \in$ $\mathbb{Z} \zeta(s)+\mathbb{Z}$ для $s$ четного и $D_{n}^{s} J_{s, n} \in \mathbb{Z} \zeta(s)+\mathbb{Z}$ для $s$ нечетного, высказанная Васильевым в его предыдущей работе [141]. Одним из первых этапов в положительном решении гипотезы Васильева (36) стал следующий частичный (с точностью до умножения на дополнительный множитель $2 D_{n}$ ) результат [166], [171], [173].

Теорема 3. Для каждого иелого $s \geqslant 2 u n=0,1,2, \ldots$ справедливо тождество

$$
J_{s, n}=F_{s, n}
$$

əəe

$$
F_{s, n}=\left.n !^{s-1} \sum_{\nu=1}^{\infty}(-1)^{(s+1)(t+n+1)}\left(t+\frac{n}{2}\right) \frac{\prod_{j=1}^{n}(t-j) \cdot \prod_{j=1}^{n}(t+n+j)}{\prod_{j=0}^{n}(t+j)^{s+1}}\right|_{t=\nu} .
$$

Как следствие, имеют место включения

$$
\begin{gathered}
2^{s-1} D_{n}^{s+1} J_{s, n} \in \mathbb{Z} \zeta(s)+\mathbb{Z} \zeta(s-2)+\cdots+\mathbb{Z} \zeta(4)+\mathbb{Z} \zeta(2)+\mathbb{Z} \text { для s четного }, \\
2 D_{n}^{s+1} J_{s, n} \in \mathbb{Z} \zeta(s)+\mathbb{Z} \zeta(s-2)+\cdots+\mathbb{Z} \zeta(5)+\mathbb{Z} \zeta(3)+\mathbb{Z} \quad \text { для s нечетного. }
\end{gathered}
$$

Отметим, что ряд (38) в точности совпадает с рядом (32) при нечетном $s$ и $r=1$, так что тождество (37) означает совпадение интегральной конструкции $\mathbb{Q}$-линейных форм от дзета-значений с конструкцией из [116].

Ряды Болла (24) и Ривоаля (32) хорошо известны в теории обобщенных гипергеометрических функций [10], [13], [130]. Формально, гипергеометрическая функция определяется рядом (1); условие

$$
\operatorname{Re}\left(a_{1}+a_{2}+\cdots+a_{m}\right)<\operatorname{Re}\left(b_{2}+\cdots+b_{m}\right)
$$

обеспечивает сходимость ряда (1) в области $|z| \leqslant 1$ (см., например, [13; § 2.1]). Важную роль в анализе гипергеометрических рядов играют формулы суммирования и преобразования. В качестве примеров укажем формулу суммирования Пфаффа-Заальшютца

$$
{ }_{3} F_{2}\left(\begin{array}{c|c}
-n, a, b \\
c, 1+a+b-c-n
\end{array} \mid 1\right)=\frac{(c-a)_{n}(c-b)_{n}}{(c)_{n}(c-a-b)_{n}}
$$

( $n$ - неотрицательное целое; см., например, [130; с. 49, формула (2.3.1.3)]), предельный случай теоремы Дугалла

$$
\begin{aligned}
& { }_{5} F_{4}\left(\begin{array}{cccc}
a, 1+\frac{1}{2} a, & b, & c, & d \\
\frac{1}{2} a, & 1+a-b, & 1+a-c, & 1+a-d
\end{array} \mid 1\right) \\
& =\frac{\Gamma(1+a-b) \Gamma(1+a-c) \Gamma(1+a-d) \Gamma(1+a-b-c-d)}{\Gamma(1+a) \Gamma(1+a-b-c) \Gamma(1+a-b-d) \Gamma(1+a-c-d)}
\end{aligned}
$$


(см. [13; §4.4]), а также преобразование Уиппла

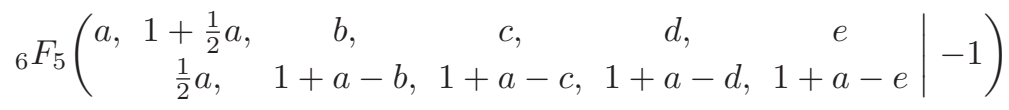

$$
\begin{aligned}
& =\frac{\Gamma(1+a-d) \Gamma(1+a-e)}{\Gamma(1+a) \Gamma(1+a-d-e)} \cdot{ }_{3} F_{2}\left(\begin{array}{c|c}
1+a-b-c, d, e \\
1+a-b, 1+a-c
\end{array} \mid 1\right)
\end{aligned}
$$

(см. [147] и [13; §4.4]). Кроме того, для гипергеометрических функций известно множество интегральных представлений [13], [130], из которых мы отметим классический интеграл Эйлера-Похгаммера для гауссовой функции $(m=1)$

$$
{ }_{2} F_{1}\left(\begin{array}{c|c}
a, b & z \\
c & z
\end{array}\right)=\frac{\Gamma(c)}{\Gamma(b) \Gamma(c-b)} \int_{0}^{1} t^{b-1}(1-t)^{c-b-1}(1-z t)^{-a} \mathrm{~d} t
$$

в случае $\operatorname{Re} c>\operatorname{Re} b>0$ (см., например, [130; с. 20, формула (1.6.6)]). Формула (44) справедлива при $|z|<1$, а также при любом $z \in \mathbb{C}$, если $а$ является неположительным целым.

В работе [148] Ф. Уиппл назвал вполне уравновешенными гипергеометрические ряды, удовлетворяющие условию

$$
a_{1}+1=a_{2}+b_{2}=\cdots=a_{m}+b_{m} ;
$$

известные преобразования (например, (42) и (43)) относятся, как правило, именно к таким рядам. Особую роль среди вполне уравновешенных гипергеометрических рядов играют ряды совершенно уравновешенные, для которых выполнено дополнительное условие

$$
a_{2}=\frac{1}{2} a_{1}+1, \quad b_{2}=\frac{1}{2} a_{1} ;
$$

обзор истории вполне и совершенно уравновешенных рядов и их приложений в различных областях математики приводится в [9]. Ряд (38) (равно как и ряд (32)) является совершенно уравновешенным:

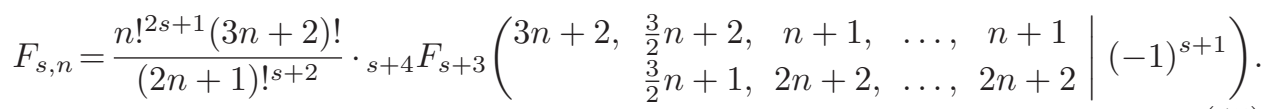

Теорема 3 является следствием общего результата [166], [171] о представлении совершенно уравновешенного ряда в виде кратного интеграла.

Именно с помощью теоремы 3 в работе [79] гипотеза Васильева (36) была решена полностью. Использованная при этом методика [79] основана на представлении сумм (45) в виде кратных гипергеометрических рядов и идейно опирается на работы [142] и [171]. Несмотря на это, техническая реализация этих идей потребовала от авторов [79] большой вычислительной работы. Для рядов (45) возможны и другие представления в виде кратных интегралов сорокинского типа (в [133], [134] содержатся теоретико-числовые приложения подобных интегралов); соответствующие теоремы о преобразовании кратных интегралов установлены С. А. Злобиным [156], [157]. В настоящее время имеется множество работ, обсуждающих представление кратных интегралов специального вида как линейных форм от дзета-значений и полилогарифмов. Эта 
тематика уже заслуживает самостоятельного обзора; мы ограничимся здесь лишь ссылками на работы [158] и [174], непосредственно связанные с другими обобщениями интегралов Бэйкерса (22) и (30).

Отметим также, что использованный в [79] гипергеометрический аппарат общее преобразование Эндрюса [8] для обрывающихся совершенно уравновешенных рядов - позволил К. Краттенталеру и Т. Ривоалю [80] дать иное доказательство теоремы из [166], [171] (и, в частности, теоремы 3). Удивительно, что это преобразование, изначально полученное для $q$-базисных гипергеометрических рядов в связи с приложениями к теории разбиений - обобщениями знаменитых формул Роджерса-Рамануджана, - нашло вторую жизнь в арифметических задачах для дзета-значений. Помимо [79], [80], здесь следует привести работы [81], [175], а также решение проблемы А. Шмидта, которое мы обсуждаем в некоторых деталях далее.

Шмидт в [127] обратил внимание на тот факт, что с последовательностью чисел Апери $\left\{u_{n}\right\}_{n=0,1, \ldots}$ (19) связано удивительное обстоятельство. Именно, если определить числа $\left\{c_{k}\right\}_{k=0,1, \ldots}$ последовательно с помощью равенств

$$
u_{n}=\sum_{k=0}^{n}\left(\begin{array}{l}
n \\
k
\end{array}\right)\left(\begin{array}{c}
n+k \\
k
\end{array}\right) c_{k}, \quad n=0,1,2, \ldots
$$

то эти числа являются целыми. (Явные формулы

$$
c_{n}=\left(\begin{array}{c}
2 n \\
n
\end{array}\right)^{-1} \sum_{k=0}^{n}(-1)^{n-k} \frac{2 k+1}{n+k+1}\left(\begin{array}{c}
2 n \\
n-k
\end{array}\right) u_{k}, \quad n=0,1,2, \ldots
$$

показывают, что ожидаемыми являются включения $D_{n} c_{n} \in \mathbb{Z}$.) Позднее сам Шмидт [128] и независимо Ф. Штрель [136] получили следующее явное выражение:

$$
c_{n}=\sum_{j=0}^{n}\left(\begin{array}{l}
n \\
j
\end{array}\right)^{3}=\sum_{j}\left(\begin{array}{l}
n \\
j
\end{array}\right)^{2}\left(\begin{array}{c}
2 j \\
n
\end{array}\right), \quad n=0,1,2, \ldots,
$$

экспериментально обнаруженное В. Дойбером, В. Тумзером и Б. Войтом. На самом деле, Штрель в [136] использовал соответствующее тождество

$$
\sum_{k=0}^{n}\left(\begin{array}{l}
n \\
k
\end{array}\right)^{2}\left(\begin{array}{c}
n+k \\
k
\end{array}\right)^{2}=\sum_{k=0}^{n}\left(\begin{array}{l}
n \\
k
\end{array}\right)\left(\begin{array}{c}
n+k \\
k
\end{array}\right) \sum_{j=0}^{k}\left(\begin{array}{l}
k \\
j
\end{array}\right)^{3}
$$

в качестве модели для иллюстрации различной техники доказательства биномиальных тождеств. Удивительным является тот факт, что последовательность (46) изучалась Ж. Франелем [60] еще в конце 19-го века: он доказал, что она удовлетворяет полиномиальной рекурсии

$$
(n+1)^{2} c_{n+1}-\left(7 n^{2}+7 n+2\right) c_{n}-8 n^{2} c_{n-1}=0 .
$$

Шмидт в [127] отметил, что по всей видимости феномен целочисленности, связанный с последовательностями чисел Апери и Франеля, выполняется в общей ситуации, - предположение, полностью доказанное в [176], [177]. 
Теорема 4. Пусть для любого иелого $r \geqslant 2$ числовая последовательность $\left\{c_{k}^{(r)}\right\}_{k=0,1, \ldots}$, не зависящая от параметра $n$, определяется равенством

$$
\sum_{k=0}^{n}\left(\begin{array}{l}
n \\
k
\end{array}\right)^{r}\left(\begin{array}{c}
n+k \\
k
\end{array}\right)^{r}=\sum_{k=0}^{n}\left(\begin{array}{l}
n \\
k
\end{array}\right)\left(\begin{array}{c}
n+k \\
k
\end{array}\right) c_{k}^{(r)}, \quad n=0,1,2, \ldots
$$

Тогда все числа $c_{k}^{(r)}$ являются иелыми.

Используя алгоритм Цайльбергера созидательного телескопирования [105], Штрель доказал в [136] целочисленность $c_{k}^{(r)}$ в случае $r=3$. Задача Шмидта была позднее сформулирована в книге [64] (упражнение 114 на с. 286) с указанием, что Г. Уилф доказал включения $c_{n}^{(r)} \in \mathbb{Z}$ для любого $r$, но только при $n \leqslant 9$. Полное доказательство теоремы 4 использует как гипергеометрическую переформулировку проблемы из [136], так и упомянутое выше преобразование Эндрюса обрывающихся совершенно уравновешенных рядов [8].

1.3. Совместные приближения к $\zeta(2)$ и $\zeta(3)$. В данном параграфе мы рассмотрим три гипергеометрические конструкции совместных рациональных приближений к $\zeta(2)$ и $\zeta(3)$. Цель этого экскурса - не только продемонстрировать гипергеометрическую технику в действии, но и показать, как на первый взгляд различные ряды приводят к одним и тем же числовым приближениям. Похожий феномен совпадения ряда Гутника-Нестеренко (23) и ряда Болла (24) был нами уже упомянут в $§ 1.1$. Приводимые далее конструкции зависят от целого возрастающего параметра $n$.

Следуя [167], рассмотрим вначале рациональные функции

$$
R_{n}(t)=-\frac{n !^{2} \prod_{j=1}^{n}(t-j)}{\prod_{j=0}^{n}(t+j)^{3}}, \quad R_{n}^{\prime}(t)=\frac{n !^{2} \prod_{j=0}^{n}(t-j)}{\prod_{j=0}^{n}(t+j)^{3}}
$$

и отвечающие им гипергеометрические ряды

$$
\begin{aligned}
& r_{n}=\left.\sum_{k=1}^{\infty} R_{n}(t)\right|_{t=\nu}=q_{n} \zeta(3)+p_{n} \zeta(2)-s_{n}, \\
& r_{n}^{\prime}=\left.\sum_{k=1}^{\infty} R_{n}^{\prime}(t)\right|_{t=\nu}=q_{n}^{\prime} \zeta(3)+p_{n}^{\prime} \zeta(2)-s_{n}^{\prime},
\end{aligned}
$$

где

$$
q_{n}, q_{n}^{\prime} \in \mathbb{Z}, \quad D_{n} p_{n}, D_{n} p_{n}^{\prime} \in \mathbb{Z}, \quad D_{n}^{3} s_{n}, D_{n}^{3} s_{n}^{\prime} \in \mathbb{Z} .
$$

Используя стандартное исключение, мы приходим к линейным формам

$$
\begin{gathered}
q_{n} r_{n}^{\prime}-q_{n}^{\prime} r_{n}=\left(q_{n} p_{n}^{\prime}-q_{n}^{\prime} p_{n}\right) \zeta(2)-\left(q_{n} s_{n}^{\prime}-q_{n}^{\prime} s_{n}\right)=u_{n} \zeta(2)-v_{n}, \\
p_{n}^{\prime} r_{n}-p_{n} r_{n}^{\prime}=\left(q_{n} p_{n}^{\prime}-q_{n}^{\prime} p_{n}\right) \zeta(3)-\left(p_{n}^{\prime} s_{n}-p_{n} s_{n}^{\prime}\right)=u_{n} \zeta(3)-w_{n},
\end{gathered}
$$

где согласно (48)

$$
D_{n} u_{n} \in \mathbb{Z}, \quad D_{n}^{3} v_{n} \in \mathbb{Z}, \quad D_{n}^{4} w_{n} \in \mathbb{Z} .
$$

Вторая конструкция [180] основана на использовании рациональной функции

$$
\widetilde{R}_{n}(t)=\frac{((t-1)(t-2) \cdots(t-n))^{3}}{n !^{2} \cdot t(t+1) \cdots(t+n)} .
$$


В этом случае гипергеометрические приближения первых трех полилогарифмов даются рядами

$$
\begin{aligned}
& \widetilde{r}_{n}(z)=\left.\sum_{\nu=1}^{\infty} z^{\nu} \widetilde{R}_{n}(t)\right|_{t=\nu}=\widetilde{u}_{n}(z) \operatorname{Li}_{1}(z)-\widetilde{s}_{n}(z), \\
& \widetilde{r}_{n}^{\prime}(z)=-\left.\sum_{\nu=1}^{\infty} z^{\nu} \frac{\mathrm{d} \widetilde{R}_{n}(t)}{\mathrm{d} t}\right|_{t=\nu}=\widetilde{u}_{n}(z) \operatorname{Li}_{2}(z)-\widetilde{v}_{n}(z), \\
& \widetilde{r}_{n}^{\prime \prime}(z)=\left.\frac{1}{2} \sum_{\nu=1}^{\infty} z^{\nu} \frac{\mathrm{d}^{2} \widetilde{R}_{n}(t)}{\mathrm{d} t^{2}}\right|_{t=\nu}=\widetilde{u}_{n}(z) \operatorname{Li}_{3}(z)-\widetilde{w}_{n}(z),
\end{aligned}
$$

где

$$
\widetilde{u}_{n}(z)=(-1)^{n} \sum_{k=0}^{n}\left(\begin{array}{c}
n \\
k
\end{array}\right)\left(\begin{array}{c}
n+k \\
k
\end{array}\right)^{3}\left(-\frac{1}{z}\right)^{k}
$$

и

$$
\begin{gathered}
z_{1}^{n} \widetilde{u}_{n}(z) \in \mathbb{Z}, \quad\left(z_{1} z_{2}\right)^{n} D_{n} \widetilde{s}_{n}(z) \in \mathbb{Z}, \\
\left(z_{1} z_{2}\right)^{n} D_{n} D_{2 n} \widetilde{v}_{n}(z) \in \mathbb{Z}, \quad\left(z_{1} z_{2}\right)^{n} D_{n} D_{2 n}^{2} \widetilde{w}_{n}(z) \in \mathbb{Z},
\end{gathered}
$$

а $z_{1}$ и $z_{2}$ означают соответственно знаменатели чисел $1 / z$ и $z /(1-z)$.

В пределе $z \rightarrow 1$ мы получаем

$$
\widetilde{r}_{n}^{\prime}(1)=\widetilde{u}_{n} \zeta(2)-\widetilde{v}_{n}, \quad \widetilde{r}_{n}^{\prime \prime}(1)=\widetilde{u}_{n} \zeta(3)-\widetilde{w}_{n}, \quad n=0,1, \ldots,
$$

где коэффициенты $\widetilde{u}_{n}=\widetilde{u}_{n}(1), \widetilde{v}_{n}=\widetilde{v}_{n}(1)$ и $\widetilde{w}_{n}=\widetilde{w}_{n}(1)$ в соответствии с $(50)$ и (51) удовлетворяют

$$
\widetilde{u}_{n} \in \mathbb{Z}, \quad D_{n} D_{2 n} \widetilde{v}_{n} \in \mathbb{Z}, \quad D_{n} D_{2 n}^{2} \widetilde{w}_{n} \in \mathbb{Z} .
$$

Наконец, возьмем рациональную функцию

$$
\widetilde{\widetilde{R}}_{n}(t)=\frac{(t-1)(t-2) \cdots(t-n) \cdot(2 t-1)(2 t-2) \cdots(2 t-n)}{(t(t+1)(t+2) \cdots(t+n))^{2}}
$$

и рассмотрим два ряда:

$$
\begin{aligned}
\left.\frac{1}{2} \sum_{\nu=1}^{\infty}(-1)^{\nu-1} R_{n}(t)\right|_{t=\nu / 2} & =\widetilde{\widetilde{u}}_{n} \zeta(2)-\widetilde{\widetilde{v}}_{n} \\
-\left.\frac{1}{2} \sum_{\nu=1}^{\infty} \frac{\mathrm{d} R_{n}(t)}{\mathrm{d} t}\right|_{t=\nu} & =\widetilde{\widetilde{u}}_{n} \zeta(3)-\widetilde{\widetilde{w}}_{n}
\end{aligned}
$$

Явные формулы для этих приближений приводят к

$$
\begin{gathered}
\widetilde{\widetilde{u}}_{n}=\sum_{k=0}^{n}\left(\begin{array}{c}
n \\
k
\end{array}\right)^{2}\left(\begin{array}{c}
n+k \\
n
\end{array}\right)\left(\begin{array}{c}
n+2 k \\
n
\end{array}\right) \in \mathbb{Z}, \quad \text { при } n=0,1,2, \ldots . \\
D_{2 n}^{2} \widetilde{\widetilde{v}}_{n} \in \mathbb{Z}, \quad D_{n}^{3} \widetilde{\widetilde{w}}_{n} \in \mathbb{Z},
\end{gathered}
$$


Теорема 5. При $n=0,1,2, \ldots$ справедливы следующие равенства:

$$
\left(\begin{array}{c}
2 n \\
n
\end{array}\right)^{-1} u_{n}=\widetilde{u}_{n}=\widetilde{\widetilde{u}}_{n}, \quad\left(\begin{array}{c}
2 n \\
n
\end{array}\right)^{-1} v_{n}=\widetilde{v}_{n}=\widetilde{\widetilde{v}}_{n}, \quad\left(\begin{array}{c}
2 n \\
n
\end{array}\right)^{-1} w_{n}=\widetilde{w}_{n}=\widetilde{\widetilde{w}}_{n} .
$$

Иными словами, три гипергеометрические конструкиии дают одну и ту же последовательность совместных рачиональных приближений $\kappa 1, \zeta(2)$ и $((3)$.

Из теоремы 5 и включений (48), (52), (53) легко выводится, что

$$
\widetilde{u}_{n} \in \mathbb{Z}, \quad D_{n} D_{2 n} \widetilde{v}_{n} \in \mathbb{Z}, \quad D_{n}^{3} \widetilde{w}_{n} \in \mathbb{Z} \quad \text { при } \quad n=0,1,2, \ldots
$$

Теорема 5 может быть доказана применением определенных гипергеометрических тождеств. Однако более простой способ (использованный в [167] и [180]) основан на алгоритме созидательного телескопирования. В самом деле, последовательности (54) удовлетворяют полиномиальным рекуррентным соотношениям третьего порядка типа Апери

$$
\begin{aligned}
& 2\left(946 n^{2}-731 n+153\right)(2 n+1)(n+1)^{3} u_{n+1} \\
& -2\left(104060 n^{6}+127710 n^{5}+12788 n^{4}-34525 n^{3}-8482 n^{2}+3298 n+1071\right) u_{n} \\
& +2\left(3784 n^{5}-1032 n^{4}-1925 n^{3}+853 n^{2}+328 n-184\right) n u_{n-1} \\
& -\left(946 n^{2}+1161 n+368\right) n(n-1)^{3} u_{n-2}=0, \quad n=2,3, \ldots,
\end{aligned}
$$

с начальными данными

$$
\begin{gathered}
\widetilde{u}_{0}=1, \quad \widetilde{u}_{1}=7, \quad \widetilde{u}_{2}=163 \\
\widetilde{v}_{0}=0, \quad \widetilde{v}_{1}=\frac{23}{2}, \quad \widetilde{v}_{2}=\frac{2145}{8}, \quad \widetilde{w}_{0}=0, \quad \widetilde{w}_{1}=\frac{17}{2}, \quad \widetilde{w}_{2}=\frac{3135}{16} .
\end{gathered}
$$

Дополнительно к этому

$$
\begin{gathered}
\limsup _{n \rightarrow \infty}\left|\widetilde{r}_{n}^{\prime}\right|^{1 / n}=\limsup _{n \rightarrow \infty}\left|\widetilde{r}_{n}^{\prime \prime}\right|^{1 / n}=\left|\lambda_{1,2}\right|=0.067442248 \ldots, \\
\lim _{n \rightarrow \infty}\left|\widetilde{u}_{n}\right|^{1 / n}=\lim _{n \rightarrow \infty}\left|\widetilde{v}_{n}\right|^{1 / n}=\lim _{n \rightarrow \infty}\left|\widetilde{w}_{n}\right|^{1 / n}=\lambda_{3}=54.96369509 \ldots,
\end{gathered}
$$

где $\lambda_{1,2}=0.018152450 \ldots \pm i 0.064953409 \ldots$ и $\lambda_{3}$ являются корнями характеристического многочлена $4 \lambda^{3}-220 \lambda^{2}+8 \lambda-1$.

Поскольку $\log \left|\lambda_{1,2}\right|=-2.69648361 \ldots>-3$, из (55) и вышесказанного мы не можем сделать заключение об иррациональности $\zeta(2)$ или $\zeta(3)$. Однако, при рассмотрении несимметричной рациональной функции

$$
\begin{aligned}
R(t)= & R(\boldsymbol{a}, \boldsymbol{b} ; t) \\
= & \frac{\left(2 t+b_{0}\right)\left(2 t+b_{0}+1\right) \cdots\left(2 t+a_{0}-1\right)}{\left(a_{0}-b_{0}\right) !} \cdot \frac{\left(t+b_{1}\right) \cdots\left(t+a_{1}-1\right)}{\left(a_{1}-b_{1}\right) !} \\
& \quad \times \frac{\left(b_{2}-a_{2}-1\right) !}{\left(t+a_{2}\right) \cdots\left(t+b_{2}-1\right)} \cdot \frac{\left(b_{3}-a_{3}-1\right) !}{\left(t+a_{3}\right) \cdots\left(t+b_{3}-1\right)} \\
= & \frac{\left(b_{2}-a_{2}-1\right) !\left(b_{3}-a_{3}-1\right) !}{\left(a_{0}-b_{0}\right) !\left(a_{1}-b_{1}\right) !} \cdot \frac{\Gamma\left(2 t+a_{0}\right) \Gamma\left(t+a_{1}\right) \Gamma\left(t+a_{2}\right) \Gamma\left(t+a_{3}\right)}{\Gamma\left(2 t+b_{0}\right) \Gamma\left(t+b_{1}\right) \Gamma\left(t+b_{2}\right) \Gamma\left(t+b_{3}\right)}
\end{aligned}
$$


с целыми $\boldsymbol{a}$ и $\boldsymbol{b}$ такими, что

$$
\begin{gathered}
b_{1}=1<a_{1}, a_{2}, a_{3}<b_{2}, b_{3}, \quad b_{0}<a_{0} \leqslant 2 \max \left\{a_{1}, a_{2}, a_{3}\right\}, \\
a_{0}+a_{1}+a_{2}+a_{3} \leqslant b_{0}+b_{1}+b_{2}+b_{3}+2,
\end{gathered}
$$

возникает следующее любопытное приложение.

Если выбрать

$$
\begin{gathered}
a_{0}=10 n+\frac{1}{2}, \quad a_{1}=6 n+1, \quad a_{2}=7 n+1, \quad a_{3}=8 n+1, \\
b_{0}=6 n+1, \quad b_{1}=1, \quad b_{2}=13 n+2, \quad b_{3}=12 n+2,
\end{gathered}
$$

то для коэффициентов соответствующих линейных форм

$$
\begin{aligned}
& r_{n}=\left.\sum_{\nu=-10 n}^{\infty}(-1)^{\nu} R(t)\right|_{t=\nu / 2}=u_{n} \zeta(2)-v_{n}, \\
& r_{n}^{\prime}=\left.\sum_{\nu=-5 n}^{\infty} \frac{\mathrm{d} R(t)}{\mathrm{d} t}\right|_{t=\nu}=u_{n} \zeta(3)-w_{n}
\end{aligned}
$$

справедливы включения

$$
\Phi_{n}^{-1} u_{n} \in \mathbb{Z}, \quad D_{8 n} D_{16 n} \Phi_{n}^{-1} v_{n} \in \mathbb{Z}, \quad D_{8 n}^{3} \Phi_{n}^{-1} w_{n} \in \mathbb{Z},
$$

где $\Phi_{n}$ - некоторое произведение по простым числам,

$$
\lim _{n \rightarrow \infty} \frac{\log \Phi_{n}}{n}=8.48973583 \ldots .
$$

С другой стороны,

$$
\limsup _{n \rightarrow \infty} \frac{\log \left|r_{n}\right|}{n}=\limsup _{n \rightarrow \infty} \frac{\log \left|r_{n}^{\prime}\right|}{n}=-17.610428885 \ldots .
$$

Таким образом, линейные формы $r_{n}$ и $r_{n}^{\prime}$ приводят к иррациональности как $\zeta(2)$, так и $\zeta(3)$; однако, их не удается использовать для доказательства совместной $\mathbb{Q}$-линейной независимости этих чисел с 1 (общим знаменателем коэффициентов является $\left.D_{8 n}^{2} D_{16 n} \Phi_{n}^{-1}\right)$.

1.4. q-Аналоги дзета-значений. Как обычно, величины, зависящие от числа $q$ и превращающиеся в классические объекты в пределе $q \rightarrow 1$ (по крайней мере формально), называются $q$-аналогами или q-расширениями. Возможный способ q-расширить значения дзета-функции Римана выглядит следующим образом (здесь $q \in \mathbb{C},|q|<1$ ):

$$
\zeta_{q}(s)=\sum_{n=1}^{\infty} \sigma_{s-1}(n) q^{n}=\sum_{\nu=1}^{\infty} \frac{\nu^{s-1} q^{\nu}}{1-q^{\nu}}=\sum_{\nu=1}^{\infty} \frac{q^{\nu} \rho_{s}\left(q^{\nu}\right)}{\left(1-q^{\nu}\right)^{s}}, \quad s=1,2, \ldots,
$$

где $\sigma_{s-1}(n)=\sum_{d \mid n} d^{s-1}$ обозначает сумму степеней делителей, а многочлены $\rho_{s}(x) \in \mathbb{Z}[x]$ могут быть определены рекурсивно с помощью формул

$$
\rho_{1}=1 \quad \text { и } \quad \rho_{s+1}=(1+(s-1) x) \rho_{s}+x(1-x) \frac{\mathrm{d} \rho_{s}}{\mathrm{~d} x} \quad \text { при } s=1,2, \ldots .
$$


Тогда имеют место предельные соотношения

$$
\lim _{\substack{q \rightarrow 1 \\|q|<1}}(1-q)^{s} \zeta_{q}(s)=\rho_{s}(1) \cdot \zeta(s)=(s-1) ! \cdot \zeta(s), \quad s=2,3, \ldots
$$

равенство $\rho_{s}(1)=(s-1)$ ! следует из $(57)$. Определенные таким образом $q$-дзета-значения (56) приводят к ряду новых интересных задач в теории диофантовых приближений и трансцендентных чисел [168], которые являются расширениями соответствующих задач для обычных дзета-значений. Несложно показать [170], что $\zeta_{q}(s)$ трансцендентны как функции параметра $q$, а также [109], что q-дзета-значения в совокупности линейно независимы над полем $\mathbb{C}(q)$.

Для четных $s \geqslant 2$ ряды $E_{s}(q)=1-2 s \zeta_{q}(s) / B_{s}$, где $B_{s} \in \mathbb{Q}$ - числа Бернулли, известны как рядъ Эйзенштейна. Поэтому модулярное происхождение (относительно параметра $\tau=\frac{\log q}{2 \pi i}$; см. также $\S 2.3$ далее) функций $E_{4}, E_{6}, E_{8}, \ldots$ приводит к алгебраической независимости $\zeta_{q}(2), \zeta_{q}(4), \zeta_{q}(6)$ над $\mathbb{Q}[q]$, в то время как остальные четные $q$-дзета-значения являются многочленами от $\zeta_{q}(4)$ и $\zeta_{q}(6)$. В такой интерпретации следствие из теоремы Нестеренко [102] - числа $\zeta_{q}(2), \zeta_{q}(4), \zeta_{q}(6)$ алгебраччески независимь над $\mathbb{Q}$ для алгебраического $q$, $0<|q|<1,-$ является полным $q$-расширением следствия из теоремы Линдемана [90], согласно которой значение $\zeta(2)=\pi^{2} / 6$ трансцендентно. Об арифметической природе нечетных $q$-дзета-значений известно немного. П. Эрдёш [52] доказал иррациональность числа $\zeta_{q}(1)$ (q-гармонического $\left.p я д а\right)$ в случае $q=p^{-1}$, где $p \in \mathbb{Z} \backslash\{0, \pm 1\} ;$ другие доказательства имеются в [27] и [32], а в [37] и [139] получена оценка

$$
\mu\left(\zeta_{q}(1)\right) \leqslant \frac{2 \pi^{2}}{\pi^{2}-2}=2.50828476 \ldots
$$

для показателя иррациональности $\zeta_{q}(1)$ при тех же условиях на параметр $q$. Конструкция линейных приближающих форм для $\zeta_{q}(1)$ в [37] и [139] имеет много общего с соответствующей конструкцией приближений Апери (23), (31). В связи с этим обстоятельством В. Ван Ассе сформулировал в [139] задачу о построении линейных приближающих форм для $\zeta_{q}(2)$ и $\zeta_{q}(3)$, доказывающих иррациональность этих чисел в случае $q^{-1} \in \mathbb{Z} \backslash\{0, \pm 1\}$ и переходящих при $q \rightarrow 1$ в последовательности Апери $u_{n}^{\prime} \zeta(2)-v_{n}^{\prime}$ и $u_{n} \zeta(3)-v_{n}$ соответственно (из $\S 1.1)$.

Методика изучения арифметических свойств чисел $\zeta(s), s=2,3, \ldots$, успешно переносится на случай $q$-дзета-значений. Именно, мы имеем в виду гипергеометрическую конструкцию линейных форм и арифметический метод, дополненный групповым подходом Дж. Рина и К. Виолы [112], [113], [144]. Для каждой из этих составляющих можно указать необходимые $q$-расширения, например, используя базисный гипергеометрический ряд, классическое преобразование Гейне [61] и q-арифметический метод [164] (таблица 1 содержит соответствующие параллели между обычной и $q$-арифметиками), мы улучшаем в [172] оценку (58) для показателя иррациональности $q$-гармонического ряда: $\mu\left(\zeta_{q}(1)\right) \leqslant 2.46497868 \ldots$. 


\begin{tabular}{|c|c|}
\hline обычные объекты & $q$-расширения, $p=1 / q \in \mathbb{Z} \backslash\{0, \pm 1\}$ \\
\hline числа $n \in \mathbb{Z}$ & $q$-числа $[n]_{p}=\frac{p^{n}-1}{p-1} \in \mathbb{Z}[p]$ \\
\hline простые $l \in\{2,3,5,7, \ldots\} \subset \mathbb{Z}$ & $\begin{array}{c}\text { неприводимые круговые многочлены } \\
\Phi_{l}(p)=\prod_{\substack{k=1 \\
k, l)=1}}^{l}\left(p-e^{2 \pi i k / l}\right) \in \mathbb{Z}[p] \\
\end{array}$ \\
\hline гамма-функция Эйлера $\Gamma(t)$ & $\begin{array}{c}q \text {-гамма-функция Джексона } \\
\Gamma_{q}(t)=\frac{\prod_{\nu=1}^{\infty}\left(1-q^{\nu}\right)}{\prod_{\nu=1}^{\infty}\left(1-q^{t+\nu-1}\right)}(1-q)^{1-t}\end{array}$ \\
\hline $\begin{array}{c}\text { факториал } n !=\Gamma(n+1) \\
n !=\prod_{\nu=1}^{n} \nu \in \mathbb{Z}\end{array}$ & $\begin{array}{c}q \text {-факториал }[n]_{q} !=\Gamma_{q}(n+1) \\
{[n]_{p} !=\prod_{\nu=1}^{n} \frac{p^{\nu}-1}{p-1}=p^{n(n-1) / 2}[n]_{q} ! \in \mathbb{Z}[p]}\end{array}$ \\
\hline $\operatorname{ord}_{l} n !=\left\lfloor\frac{n}{l}\right\rfloor+\left\lfloor\frac{n}{l^{2}}\right\rfloor+\cdots$ & $\operatorname{ord}_{\Phi_{l}(p)}[n]_{p} !=\left\lfloor\frac{n}{l}\right\rfloor, l=2,3,4, \ldots$ \\
\hline $\begin{aligned} D_{n} & =\operatorname{HOK}(1, \ldots, n) \\
& =\prod_{\text {простые } l \leqslant n} l^{\lfloor\log n / \log l\rfloor} \in \mathbb{Z}\end{aligned}$ & $\begin{aligned} D_{n}(p) & =\operatorname{HOK}\left([1]_{p}, \ldots,[n]_{p}\right) \\
& =\prod_{l=1}^{n} \Phi_{l}(p) \in \mathbb{Z}[p]\end{aligned}$ \\
\hline $\begin{array}{c}\text { асимптотический закон } \\
\text { распределения простых чисел } \\
\qquad \lim _{n \rightarrow \infty} \frac{\log D_{n}}{n}=1\end{array}$ & $\begin{array}{l}\text { формула Мертенса } \\
\lim _{n \rightarrow \infty} \frac{\log \left|D_{n}(p)\right|}{n^{2} \log |p|}=\frac{3}{\pi^{2}}\end{array}$ \\
\hline
\end{tabular}

ТАБлицА 1. Сравнение $q$-арифметики с обычной. Здесь $\lfloor\cdot\rfloor-$ целая часть числа и сокращение "НОК" используется для обозначения наименьшего общего кратного

Используя $q$-аналог гипергеометрического ${ }_{3} F_{2}(1)$-ряда и преобразование Холла [61], в работе [165] не только решается задача Ван Ассе для $\zeta_{q}(2)$, но и оптимизируется оценка для показателя иррациональности этого числа.

Теорема 6. Для каждого $q=1 / p, p \in \mathbb{Z} \backslash\{0, \pm 1\}$, число $\zeta_{q}(2)$ является ирраииональным с показателем иррациональности, удовлетворяющим неравенству

$$
\mu\left(\zeta_{q}(2)\right) \leqslant 4.07869374 \ldots
$$

Количественные оценки типа (59) для $\zeta_{q}(2)$ (доказывающие нелиувиллевость этой постоянной в случае $\left.q^{-1} \in \mathbb{Z} \backslash\{0, \pm 1\}\right)$ ранее не были известны, хотя, как отмечалось выше, иррациональность [49] и даже трансцендентность числа $\zeta_{q}(2)$ для любого алгебраического $q$ с условием $0<|q|<1$ следует из теоремы Нестеренко [102]. Иная интерпретация рациональных приближений к $\zeta_{q}(2)$ в [131] упростила контроль над арифметической составляющей и позволила усилить 
оценку (59):

$$
\mu\left(\zeta_{q}(2)\right) \leqslant \frac{10 \pi^{2}}{5 \pi^{2}-24}=3.89363887 \ldots
$$

Частный случай гипергеометрической конструкции работ [165], [131], именно

$$
U_{n}(q) \zeta_{q}(2)-V_{n}(q)=\left.(-1)^{n} \sum_{\nu=1}^{\infty} \frac{\prod_{j=1}^{n}\left(1-q^{j}\right) \cdot \prod_{j=1}^{n}\left(1-q^{j} T\right)}{\prod_{j=0}^{n}\left(1-q^{n+1+j} T\right)^{2}} T^{n+1}\right|_{T=q^{\nu}},
$$

позволяет доказать иррациональность числа $\zeta_{q}(2)$ в случае $q^{-1} \in \mathbb{Z} \backslash\{0, \pm 1\}$, а в пределе $q \rightarrow 1$ получаются рациональные приближения Апери (31) к $\zeta(2)$. В совместной работе [84] мы предъявляем $q$-аналог последовательности рациональных приближений (23), (24), однако это не приводит к иррациональности величины $\zeta_{q}(3)$.

Использование $q$-арифметического метода и гипергеометрической конструкции позволяет получить и другие количественные и качественные результаты для $q$-дзета-значений. Так, работа [84] содержит результат о бесконечности иррациональных чисел среди нечетных $q$-дзета-значений ( $q$-аналог теоремы Ривоаля) в случае $q^{-1} \in \mathbb{Z} \backslash\{0, \pm 1\}$; количественные результаты из [84] были немного улучшены в недавних работах [58], [78]. Особый статус получила линейная независимость (в тех же предположениях на $q$ ) значений $\zeta_{q}(1), \zeta_{q}(2)$ и 1 над $\mathbb{Q}$, как в качественной, так и количественной формах; см. на этот счет статьи [38], [107], [138], [178].

Отметим также, что один из подходов к гипотезе Римана (см. [28]) и проблемам целочисленности разложений, возникающих в теории струн (см. [45] и 2.2 далее), опирается на целозначные отношения факториалов и соответствующие производящие гипергеометрические ряды. Как показано в [146] (в большинстве случаев только гипотетически), q-сторона этого подхода приводит к q-многочленам с неотрицательными коэффициентами.

1.5. Нижняя оценка для $\left\|(3 / 2)^{k}\right\|$ и проблема Варинга. Мы не ставим цели охватить все возможные приложения гипергеометрических конструкций в арифметике. В качестве заключительного к этому разделу аккорда мы укажем еще одну задачу на стыке диофантовой и аналитической теорий чисел. На первый взгляд она не кажется связанной с теоремой Апери, но эффективные подходы к ее решению - гипергеометрическая конструкция и арифметический метод (применяющийся, в частности, для доказательства теорем 2 и 6) - убеждают в обратном.

Пусть $\lfloor\cdot\rfloor$ и $\{\cdot\}$ обозначают целую и дробную части числа соответственно. Как известно [143], неравенство $\left\{(3 / 2)^{k}\right\} \leqslant 1-(3 / 4)^{k}$ при $k \geqslant 6$ дает точную формулу $g(k)=2^{k}+\left\lfloor(3 / 2)^{k}\right\rfloor-2$ для наименьшего целого $g=g(k)$ такого, что каждое натуральное число представимо в виде суммы не более $g$ положительных $k$-х степеней (проблема Варинга). К. Малер [92] использовал обобщение Риду известной теоремы Рота, чтобы показать, что неравенство $\left\|(3 / 2)^{k}\right\| \leqslant C^{k}$, где $\|x\|=\min (\{x\}, 1-\{x\})$ - расстояние от $x \in \mathbb{R}$ до ближайшего целого, имеет лишь конечное число решений в целых $k$ для любого $C<1$. В частном случае $C=3 / 4$ получается приведенное выше значение $g(k)$ для всех $k \geqslant K$, где $K-$ 
некоторая абсолютная, но неэффективная постоянная. Таким образом, возникает вопрос о нетривиальной (т.е. $C>1 / 2$ ) и эффективной (в терминах $K$ ) оценке вида

$$
\left\|\left(\frac{3}{2}\right)^{k}\right\|>C^{k} \quad \text { для всех } \quad k \geqslant K .
$$

Первое продвижение в решении этой задачи принадлежит А. Бейкеру и Дж. Коэтсу [14]; применив эффективные оценки линейных форм от логарифмов в $p$-адическом случае, они показали справедливость $(60)$ с $C=2^{-\left(1-10^{-64}\right)}$. $\Phi$. Бэйкерс [22] существенно улучшил этот результат, доказав, что неравенство (60) выполняется с $C=2^{-0.9}=0.5358 \ldots$ при $k \geqslant K=5000$ (хотя его доказательство давало и лучший выбор $C=0.5637 \ldots$, если не заботиться о явном вычислении эффективной границы для $K$ ). Доказательство Бэйкерса основано на приближениях Паде к остатку биномиального ряда $(1-z)^{m}=$ $\sum_{n=0}^{m}\left(\begin{array}{c}m \\ n\end{array}\right)(-z)^{n}$; позднее А. Дубицкас [48] и Л. Хабсигер [74] использовали конструкцию Бэйкерса для получения оценки (60) с $C=0.5769$ и 0.5770 соответственно. Последняя работа также содержит оценку $\left\|(3 / 2)^{k}\right\|>0.57434^{k}$ при $k \geqslant 5$ на основе вычислений из [46] и [85].

Модифицируя конструкцию Бэйкерса [22], именно, рассматривая приближения Паде остатка ряда

$$
\frac{1}{(1-z)^{m+1}}=\sum_{n=0}^{\infty}\left(\begin{array}{c}
m+n \\
m
\end{array}\right) z^{n}
$$

и получая точные оценки $p$-адических порядков возникающих биномиальных коэффициентов, мы доказываем [179] неравенство

$$
\left\|\left(\frac{3}{2}\right)^{k}\right\|>0.5803^{k}=2^{-k \cdot 0.78512916 \ldots} \quad \text { для всех } k \geqslant K \text {, }
$$

где $K$ - некоторая эффективная постоянная.

Конструкция [179] позволяет также доказать оценки

$$
\begin{aligned}
& \left\|\left(\frac{4}{3}\right)^{k}\right\|>0.4914^{k}=3^{-k \cdot 0.64672207 \ldots} \quad \text { при } \quad k \geqslant K_{1}, \\
& \left\|\left(\frac{5}{4}\right)^{k}\right\|>0.5152^{k}=4^{-k \cdot 0.47839775 \ldots} \quad \text { при } \quad k \geqslant K_{2},
\end{aligned}
$$

где $K_{1}, K_{2}$ - эффективные постоянные. Наилучший результат для последовательностей $\left\|(1+1 / N)^{k}\right\|$ принадлежит М. Беннетту [17]: $\left\|(1+1 / N)^{k}\right\|>3^{-k}$ при $4 \leqslant N \leqslant k \cdot 3^{k}$. Наша оценка снизу для $\left\|(4 / 3)^{k}\right\|$ дополняет результат Беннетта [18] о порядке аддитивного базиса $\left\{1, N^{k},(N+1)^{k},(N+2)^{k}, \ldots\right\}$ в случае $N=3$ (случай $N=2$ отвечает классической проблеме Варинга); решение соответствующей задачи требует оценки $\left\|(4 / 3)^{k}\right\|>(4 / 9)^{k}$ при $k \geqslant 6$. Вопрос об эффективизации оценок (62) обсуждается в [110]. 


\section{2. Дифференциальные уравнения Калаби-Яу}

\section{1. Арифметические дифференциальные уравнения второго и} третьего порядка. Некоторые дифференциальные уравнения выглядят лучше других, по крайней мере арифметически. Для иллюстрации этого принципа рассмотрим дифференциальное уравнение

$$
\left(\theta^{2}-z\left(11 \theta^{2}+11 \theta+3\right)-z^{2}(\theta+1)^{2}\right) y=0, \quad \text { где } \quad \theta=z \frac{\mathrm{d}}{\mathrm{d} z} .
$$

Что же в нем такого особенного? Во-первых, оно имеет единственное аналитическое решение $y_{0}(z)=f(z), f(0)=1$; другое решение можно представить в виде $y_{1}(z)=f(z) \log z+g(z), g(0)=0$. Во-вторых, коэффициенты в разложении Тейлора $f(z)=\sum_{n=0}^{\infty} A_{n} z^{n}$ являются целыми числами, $f(z) \in 1+z \mathbb{Z}[[z]]$, что едва ли можно уяснить из определяющих рекуррентных соотношений

$$
(n+1)^{2} A_{n+1}-\left(11 n^{2}+11 n+3\right) A_{n}-n^{2} A_{n-1}=0 \quad \text { при } n=0,1, \ldots, \quad A_{0}=1
$$

(ср. (25)), но следует из полученного Апери [12] явного выражения

$$
A_{n}=\sum_{k=0}^{n}\left(\begin{array}{l}
n \\
k
\end{array}\right)^{2}\left(\begin{array}{c}
n+k \\
n
\end{array}\right), \quad n=0,1, \ldots
$$

Отметим, что данные числа появляются в доказательстве Апери иррациональности $\zeta(2)$. В третьих, разложение $q(z)=\exp \left(y_{1}(z) / y_{0}(z)\right)=z \exp (g(z) / f(z))$ также имеет целые коэффициенты, $q(z) \in z \mathbb{Z}[[z]]$. Это следует из того, что обратная к $z(q)$ функция

$$
z(q)=q \prod_{n=1}^{\infty}\left(1-q^{n}\right)^{5\left(\frac{n}{5}\right)},
$$

где $\left(\frac{n}{5}\right)$ обозначает символ Лежандра, лежит в $q \mathbb{Z}[[q]]$. Из формулы (66), полученной Ф. Бэйкерсом [23], следует, что $z(q)$ - модулярная функция относительно конгруэнц-подгруппы $\Gamma_{1}(5)$ группы $\mathrm{SL}_{2}(\mathbb{Z})$.

Читатель, для которого данные свойства целочисленности не являются удивительными, может попробовать отыскать другие подобные случаи, заменив дифференциальный оператор из (63) более общим

$$
D=D(a, b, c):=\theta^{2}-z\left(a \theta^{2}+a \theta+b\right)+c z^{2}(\theta+1)^{2} .
$$

При обеспечении требуемой целочисленности несложно видеть, что $a, b, c \in \mathbb{Z}$, но при общем выборе параметров второе свойство $\left(y_{0}(z)=f(z) \in 1+z \mathbb{Z}[[z]]\right)$ "практически всегда" нарушается. В действительности данная задача рассматривалась Ф. Бэйкерсом [25] и Д. Загиром [154]. В результате исчерпывающего экспериментального поиска, проведенного в [154], было получено 14 (невырожденных) примеров троек $(a, b, c) \in \mathbb{Z}^{3}$, для которых удовлетворяются как второе, так и третье свойства (целочисленность соответствующего разложения $z(q))$; последнее следует из модулярных интерпретаций $z(q)$. 


\begin{tabular}{|c|c|c|}
\hline$\#$ в [5] & $\#$ в [154] & $(a, b, c)$ \\
\hline$(\mathrm{A})$ & $\# 11$ & $(16,4,0)$ \\
$(\mathrm{B})$ & $\# 14$ & $(27,6,0)$ \\
(C) & $\# 20$ & $(64,12,0)$ \\
$(\mathrm{D})$ & & $(432,60,0)$ \\
$(\mathrm{e})$ & $\# 19$ & $\left(32,12,16^{2}\right)$ \\
$(\mathrm{h})$ & $\# 25$ & $\left(54,21,27^{2}\right)$ \\
(i) & $\# 26$ & $\left(128,52,64^{2}\right)$ \\
(j) & & $\left(864,372,432^{2}\right)$ \\
(a) & $\# 5, \mathbf{A}$ & $(7,2,-8)$ \\
(b) & $\# 9, \mathbf{D}$ & $(11,3,-1)$ \\
(c) & $\# 8, \mathbf{C}$ & $(10,3,9)$ \\
(d) & $\# 10, \mathbf{E}$ & $(12,4,32)$ \\
(f) & $\# 7, \mathbf{B}$ & $(9,3,27)$ \\
(g) & $\# 13, \mathbf{F}$ & $(17,6,72)$ \\
\hline
\end{tabular}

\begin{tabular}{|c|c|}
\hline$\#$ в $[5]$ & $(\hat{a}, \hat{b}, \hat{c})$ \\
\hline$(\beta)$ & $\left(16,8,16^{2}\right)$ \\
$(\iota)$ & $\left(27,15,27^{2}\right)$ \\
$(\vartheta)$ & $\left(64,40,64^{2}\right)$ \\
$(\kappa)$ & $\left(432,312,432^{2}\right)$ \\
& $(32,8,0)$ \\
& $(54,12,0)$ \\
& $(128,24,0)$ \\
& $(864,120,0)$ \\
$(\delta)$ & $(7,3,81)$ \\
$(\eta)$ & $(11,5,125)$ \\
$(\alpha)$ & $(10,4,64)$ \\
$(\epsilon)$ & $(12,4,16)$ \\
$(\zeta)$ & $(9,3,-27)$ \\
$(\gamma)$ & $(17,5,1)$ \\
\hline
\end{tabular}

ТАБлицА 2. Арифметические дифференциальные операторы $D(a, b, c)$ и $\hat{D}(\hat{a}, \hat{b}, \hat{c})$

Естественное обобщение такой постановки вопроса для линейных дифференциальных уравнения третьего порядка подсказывается другой последовательностью Апери, использованной им при доказательстве [12] иррациональности $\zeta(3)$. Для семейства дифференциальных операторов

$$
\widehat{D}=\widehat{D}(\hat{a}, \hat{b}, \hat{c}):=\theta^{3}-z(2 \theta+1)\left(\hat{a} \theta^{2}+\hat{a} \theta+\hat{b}\right)+\hat{c} z^{2}(\theta+1)^{3}
$$

нас будет интересовать, когда два решения $f(z) \in 1+z \mathbb{C}[[z]]$ и $f(z) \log z+g(z)$ с $g(0)=0$ соответствующего дифференциального уравнения таковы, что $f(z) \in$ $\mathbb{Z}[[z]]$ и $\exp (g(z) / f(z)) \in \mathbb{Z}[[z]]$. За исключением некоторых вырожденных случаев, мы смогли найти [6] опять же 14 троек $(\hat{a}, \hat{b}, \hat{c}) \in \mathbb{Z}^{3}$, для которых выполнены условия целочисленности; как и ранее, вторая целочисленность следует из модулярных интерпретаций. Пример Апери соответствует случаю $(\hat{a}, \hat{b}, \hat{c})=$ $(17,5,1)$. В таблице 2 перечислены найденные 14 примеров второго порядка и 14 примеров третьего порядка, а в следующей далее теореме указывается явный вид соответствия между ними. Доказательство этой теоремы, а также ее геометрическая мотивация приведены в [5; Theorem 1], где также указываются явные биномиальные выражения для аналитических решений $f(z)$.

ТеОрема 7. Предположим, что тройки $(a, b, c) u(\hat{a}, \hat{b}, \hat{c})$ связаны соотношениями

$$
\hat{a}=a, \quad \hat{b}=a-2 b \quad u \quad \hat{c}=a^{2}-4 c .
$$

Далее, для определенных в (67) и (68) дифференииальных операторов $D$ и $\widehat{D}$ через $f(z)$ и $\hat{f}(z)$ обозначим соответствующие аналитические решения уравнений $D y=0$ и $\widehat{D} y=0$ с условиями $f(0)=\hat{f}(0)=1$. Тогда

$$
f(z)^{2}=\frac{1}{1-a z+c z^{2}} \hat{f}\left(\frac{-z}{1-a z+c z^{2}}\right) .
$$


2.2. Арифметические дифференциальные уравнения четвертого и пятого порядка. Как можно обобщить рассмотренный выше вопрос нахождения "арифметически хороших" линейных дифференциальных уравнений (операторов)? В [6], [3] мы не ограничиваем форму оператора, как в (67) и (68), но рассматриваем уравнения, по крайней мере до пятого порядка, для которых выполняются следующие условия:

(i) дифференциальное уравнение фуксово, т.е. все его особые точки регулярны; дополнительно, локальные показатели при $z=0$ равны нулю;

(ii) единственное аналитическое решение $y_{0}(z)=f(z)$ с условием $f(0)=1$ в нуле имеет целые коэффициенты, $f(z) \in 1+z \mathbb{Z}[[z]]$;

(iii) решение $y_{1}(z)=f(z) \log z+g(z)$ с условием $g(0)=0$ приводит к целочисленному представлению $q(z):=\exp \left(y_{1}(z) / y_{0}(z)\right) \in z \mathbb{Z}[[z]]$.

Требование (i), известное как условие максималъной унипотентной монодромии (MUM - maximally unipotent monodromy), означает, что соответствующий дифференциальный оператор, записанный как многочлен по переменной $z$ с коэффициентами из $\mathbb{C}[[\theta]]$, имеет постоянный член $\theta^{m}$, где $m$ - порядок (степень по $\theta$ ); локальная монодромия в окрестности нуля представлена одной жордановой клеткой максимального размера. Отметим, что условие (i) гарантирует единственность $y_{0}(z)$ и $y_{1}(z)$. Условие (ii) обычно можно ослабить до $f(C z) \in 1+z \mathbb{Z}[[z]]$ при некотором натуральном $C$ (без масштабирования $z \mapsto C z$ многие из получающихся формул выглядят "более естественными"). Условие (iii) означает, что функция, обратная к $z(q)$ (так называемое зеркальное отображение), также обладает целочисленным разложением; более того, можно рассматривать $q$ как новую переменную, по крайней мере в окрестности нуля.

В действительности, в [6], [3] на дифференциальные уравнения четвертого порядка ${ }^{1}$

$$
y^{\prime \prime \prime \prime}+P y^{\prime \prime \prime}+Q y^{\prime \prime}+R y^{\prime}+S y=0
$$

нами также наложены дополнительные условия:

(iv) "условие Калаби-Яу" или "условие самодвойственности"

$$
R=\frac{1}{2} P Q-\frac{1}{8} P^{3}+Q^{\prime}-\frac{3}{4} P P^{\prime}-\frac{1}{2} P^{\prime \prime},
$$

определяющее структуру проективной группы монодромии;

(v) условие целочисленности соответствующей последовательности чисел $N_{0}, N_{1}, \ldots$, называемых в физике инстантонными числами; они возникают как коэффициенты в $q$-разложении Ламберта так называемого спаривания Юкавы

$$
\begin{aligned}
K & =K(q):=\frac{N_{0}}{y_{0}^{2}}\left(q \frac{\mathrm{d} q}{\mathrm{~d} z}\right)^{3} \exp \left(-\frac{1}{2} \int P(z) \mathrm{d} z\right) \\
& =N_{0}+\sum_{d=1}^{\infty} \frac{N_{d} d^{3} q^{d}}{1-q^{d}} .
\end{aligned}
$$

\footnotetext{
${ }^{1} \mathrm{~B}$ этом разделе символ ' означает дифференцирование по $z$.
} 
На протяжении долгого времени мы были уверены, что во всех примерах вышеупомянутые дополнительные условия (iv), (v) удовлетворяются автоматически при выполнении (i)-(iii). Однако лишь недавно нам стало известно от М. Богнера и С. Райтера [29], что дифференциальный оператор

$$
\theta^{4}-8 z(2 \theta+1)^{2}\left(5 \theta^{2}+5 \theta+2\right)+192 z^{2}(2 \theta+1)(2 \theta+3)(3 \theta+2)(3 \theta+4)
$$

удовлетворяет условиям (i)-(iv), но при этом условие (v) по всей видимости нарушается. Следовательно, уравнения Калаби-Яу четвертого порядка характеризуются всеми условиями (i)-(v). Более того, антисимметричный квадрат любого уравнения Калаби-Яу четвертого порядка является линейным дифференциальным уравнением пятого порядка; в случае, если новое уравнение удовлетворяет условиям (i)-(iii) (а это всегда так во всех известных примерах, хотя строго данное утверждение можно установить лишь в нескольких частных случаях), такое уравнение мы называем уравнением Калаби-Яу пятого порядка.

В результате проведенных экспериментальных поисков [6], [3] нами найдено более 400 таких дифференциальных уравнений. Соответствующие дифференциальные операторы являются операторами типа Калаби-Яy, поскольку некоторые из таких примеров можно идентифицировать с дифференциальными уравнениями Пикара-Фукса для периодов однопараметрических семейств многообразий Калаби-Яу. Для элемента нашей таблицы из [3] проверка условий (i) и (iv) тривиальна, условие (ii) обычно следует из явного выражения коэффициентов $f(z)$ (в случае наличия такового), а (iii) может в ряде случаев быть установлено с использованием $p$-адической техники Дворка. Существенный прогресс в этом направлении получен в недавних работах К. Краттенталера и Т. Ривоаля [82], [83].

Простейшие примеры дифференциальных уравнений Калаби-Яу связаны с общим гипергеометрическим дифференциальным уравнением (2) порядка $m=4$ (и $m=5$ ), которому удовлетворяет гипергеометрический ряд (1). Уравнение (2) имеет (наименьшую возможную) степень 1 по $z$, а из условия (i) вытекает $b_{2}=\cdots=b_{m}=1$. Это дает основание считать уравнения Калаби-Яу и их аналитические решения естественным арифметическим обобщением гипергеометрических уравнений и рядов.

Согласно стандартным гипотезам (см., например, [7]) все полученные в [3] дифференциальные операторы Калаби-Яу должны иметь "геометрическое происхождение", иными словами, должны соответствовать (как неполные частные локальных систем) делителям уравнений Пикара-Фукса, решения которых - интегралы периодов некоторых семейств многообразий над проективной прямой.

В работе [5] приводится много явных алгебраических преобразований, связывающих дифференциальные уравнения Калаби-Яу с их решениями и которые можно рассматривать как обобщения теоремы 7 на бо́льшие размерности; примерами служат преобразование (3) из введения и рассматриваемое далее преобразование (98). В [5] указывается простой рецепт проверки того, что два уравнения Калаби-Яу связаны некоторым алгебраическим преобразованием, и дается способ получения явного вида такового. 
Другое арифметическое свойство дифференциальных уравнений КалабиЯу, впервые рассмотренное в [4] в максимально общей постановке, связано с вычислением соответствующих пределов Апери. Понятие предела Апери восходит к работе Апери об иррациональности значений $\zeta(2)$ и $\zeta(3)$, о которой мы уже говорили в $\S 1.1$, и рассматривается в ряде работ в связи с арифметическими дифференциальными уравнениями второго и третьего порядка и их модулярными параметризациями; основными ссылками по этому вопросу являются [23], [24], [150], [154]. Для заданного дифференциального оператора Калаби-Яу D предел Апери может быть определен следующим образом. Рассмотрим аналитическое решение $y_{0}(z)=f(z) \in 1+z \mathbb{Z}[[z]]$ уравнения $D y=0$ и (единственное) аналитическое решение $\tilde{f}(z) \in z+z^{2} \mathbb{Q}[[z]]$ связанного с ним неоднородного дифференциального уравнения $D \tilde{y}=z$. Соответствующие разложения имеют вид

$$
f(z)=1+\sum_{n=1}^{\infty} u_{n} z^{n} \quad \text { и } \quad \tilde{f}(z)=z+\sum_{n=2}^{\infty} v_{n} z^{n} .
$$

Тогда предел Апери $\mathrm{Ap}(D)$ определяется как

$$
\operatorname{Ap}(D):=\lim _{n \rightarrow \infty} \frac{v_{n}}{u_{n}} .
$$

Введение этого понятия мотивируется тем, что из результатов Апери (25)-(29) и (16)-(21) следует, что

$$
\operatorname{Ap}(D(11,3,-1))=\frac{\zeta(2)}{5} \quad \text { и } \quad \operatorname{Ap}(\widehat{D}(17,5,1))=\frac{\zeta(3)}{6}
$$

(ср. с таблицей 2). В [4] на основании многих доказанных там и в [150] примеров было замечено, что пределы Апери дифференциальных операторов Калаби-Яу оказываются равными значениям некоторых $L$-рядов, связанных с квадратичными характерами и эллиптическими кривыми.

2.3. Семейство квинтик Калаби-Яу. В этом параграфе мы обсудим простейшие проявления скрытой в дифференциальных уравнениях Калаби-Яу геометрии.

Пусть $M_{z}$ - семейство трехмерных многообразий Калаби-Яу, параметризованных комплексной переменной $z \in \mathbb{P}^{1}(\mathbb{C})$. Тогда периоды единственной голоморфной дифференциальной 3 -формы на $M_{z}$ удовлетворяют некоторому линейному дифференциальному уравнению, называемому дифференциальным уравнением Пикара-Фукса семейства $M_{z}$. В случае, когда число Ходжа $h^{2,1}$ равно 1, дифференциальное уравнение Пикара-Фукса имеет четвертый порядок. По-видимому, один из наиболее известных примеров дается семейством трехмерных квинтик [40] в $\mathbb{P}^{4}$ :

$$
x_{1}^{5}+x_{2}^{5}+x_{3}^{5}+x_{4}^{5}+x_{5}^{5}-z^{-1 / 5} x_{1} x_{2} x_{3} x_{4} x_{5}=0,
$$

для которого дифференциальное уравнение Пикара-Фукса имеет вид

$$
\theta^{4} y-5 z(5 \theta+1)(5 \theta+2)(5 \theta+3)(5 \theta+4) y=0, \quad \theta=z \frac{\mathrm{d}}{\mathrm{d} z} .
$$


Данное семейство представляет собой одно из четырнадцати семейств трехмерных многообразий Калаби-Яу [3], для которых дифференциальные уравнения Пикара-Фукса являются гипергеометрическими.

Предваряя обсуждение свойств этого и других примеров специальных арифметических дифференциальных уравнений, мы вначале рассмотрим случаи более простой природы и их классические связи с теорией модулярных и гипергеометрических функций.

Хорошо известен результат о том, что решение

$$
{ }_{2} F_{1}\left(\begin{array}{c|c}
\frac{1}{2}, & \frac{1}{2} \\
1 & z
\end{array}\right)
$$

дифференциального уравнения Пикара-Фукса

$$
\theta^{2} y-\frac{z}{4}(2 \theta+1)^{2} y=0
$$

отвечающего семейству эллиптических кривых (т.е. одномерных многообразий)

$$
E_{z}: y^{2}=x(x-1)(x-z)
$$

удовлетворяет равенству

$$
{ }_{2} F_{1}\left(\begin{array}{c|c}
\frac{1}{2}, \frac{1}{2} & \frac{\vartheta_{2}^{4}}{\vartheta_{3}^{4}}
\end{array}\right)=\vartheta_{3}^{2},
$$

где $\vartheta_{2}(\tau)=\sum_{n \in \mathbb{Z}} e^{\pi i \tau(n+1 / 2)^{2}}$ и $\vartheta_{3}(\tau)=\sum_{n \in \mathbb{Z}} e^{\pi i \tau n^{2}}-$ модулярные формы веса $1 / 2$. Иными словами, в подходящих обозначениях $z$ становится модулярной функцией, а голоморфное в нуле решение дифференциального уравнения становится модулярной формой веса 1 относительно конгруэнц-подгруппы $Г(2)$ группы $\mathrm{SL}_{2}(\mathbb{Z})$. Сходным образом, решение

$$
{ }_{3} F_{2}\left(\begin{array}{c|c}
\frac{1}{4}, \frac{1}{2}, \frac{3}{4} & 256 z \\
1,1 &
\end{array}\right)
$$

дифференциального уравнения Пикара-Фукса

$$
\theta^{3} y-4 z(4 \theta+1)(4 \theta+2)(4 \theta+3) y=0
$$

для семейства $K 3$-поверхностей (т.е. для двумерных многообразий Калаби-Яу)

$$
K_{z}: x_{1}^{4}+x_{2}^{4}+x_{3}^{4}+x_{4}^{4}-z^{-1 / 4} x_{1} x_{2} x_{3} x_{4}=0
$$

может быть интерпретировано как модулярная форма веса 2 относительно $\Gamma_{0}(2)$. Эти примеры дают основания полагать, что голоморфное в нуле решение уравнения (75) может быть интерпретировано как обобщенная модулярная (или автоморфная) форма.

Чтобы убедиться в том, что решения уравнения (75) и их производные порождают "более богатую” алгебраическую структуру, отметим [94], [104], что в классическом случае модулярная форма или функция $f(\tau)$, а также 
две ее последовательные производные $f^{\prime}(\tau)$ и $f^{\prime \prime}(\tau)$ алгебраически независимы с $q=e^{2 \pi i \tau}$ над полем $\mathbb{C}(\tau)$, в то время как все старшие производные алгебраичны над полем $\mathbb{C}\left(f(\tau), f^{\prime}(\tau), f^{\prime \prime}(\tau)\right)$. $\mathrm{K}$ примеру, кольцо квазимодулярных форм $\mathbb{C}\left[E_{2}(\tau), E_{4}(\tau), E_{6}(\tau)\right]$, где

$$
\begin{gathered}
E_{2}(\tau)=1-24 \sum_{n=1}^{\infty} \frac{n q^{n}}{1-q^{n}}, \\
E_{4}(\tau)=1+240 \sum_{n=1}^{\infty} \frac{n^{3} q^{n}}{1-q^{n}}, \quad E_{6}(\tau)=1-504 \sum_{n=1}^{\infty} \frac{n^{5} q^{n}}{1-q^{n}},
\end{gathered}
$$

дифференциально устойчиво [111], [169]:

$$
\begin{gathered}
\dot{E}_{2}=\frac{1}{12}\left(E_{2}^{2}-E_{4}\right), \quad \dot{E}_{4}=\frac{1}{3}\left(E_{2} E_{4}-E_{6}\right), \quad \dot{E}_{6}=\frac{1}{2}\left(E_{2} E_{6}-E_{4}^{2}\right), \\
\text { где } \quad \dot{E}:=\frac{1}{2 \pi i} \frac{\mathrm{d} E}{\mathrm{~d} \tau}=q \frac{\mathrm{d} E}{\mathrm{~d} q} .
\end{gathered}
$$

Первый пример нелинейного дифференциального уравнения седьмого порядка для спаривания Юкавы был представлен в [89], при этом в [161] было показано, что такое алгебраическое дифференциальное уравнение с коэффициентами из $\mathbb{C}(q)$ имеет минимальный порядок. Отметим, что приведенное в [89] уравнение очень длинное, и лишь недавно Х. Мовасати [99] удалось построить очень элегантную систему нелинейных дифференциальных уравнений для семейства трехмерных квинтик (74) и отвечающего ему уравнения Пикара-Фукса (75), которое напоминает систему Рамануджана (77):

$$
\begin{aligned}
& \dot{T}_{0}=\frac{1}{T_{5}}\left(\frac{6}{5} T_{0}^{5}+\frac{1}{3125} T_{0} T_{3}-\frac{1}{5} T_{4}\right) \\
& \dot{T}_{1}=\frac{1}{T_{5}}\left(-125 T_{0}^{6}+T_{0}^{4} T_{1}+125 T_{0} T_{4}+\frac{1}{3125} T_{1} T_{3}\right) \\
& \dot{T}_{2}=\frac{1}{T_{5}}\left(-1875 T_{0}^{7}-\frac{1}{5} T_{0}^{5} T_{1}+2 T_{0}^{4} T_{2}+1875 T_{0}^{2} T_{4}+\frac{1}{5} T_{1} T_{4}+\frac{2}{3125} T_{2} T_{3}\right), \\
& \dot{T}_{3}=\frac{1}{T_{5}}\left(-3125 T_{0}^{8}-\frac{1}{5} T_{0}^{5} T_{2}+3 T_{0}^{4} T_{3}+3125 T_{0}^{3} T_{4}+\frac{1}{5} T_{2} T_{4}+\frac{3}{3125} T_{3}^{2}\right), \\
& \dot{T}_{4}=\frac{1}{T_{5}}\left(5 T_{0}^{4} T_{4}+\frac{1}{625} T_{3} T_{4}\right), \\
& \dot{T}_{5}=\frac{T_{6}}{T_{5}}, \\
& \dot{T}_{6}=\left(-\frac{72}{5} T_{0}^{8}-\frac{24}{3125} T_{0}^{4} T_{3}-\frac{3}{5} T_{0}^{3} T_{4}-\frac{2}{1953125} T_{3}^{2}\right)+\frac{T_{6}}{T_{5}}\left(12 T_{0}^{4}+\frac{2}{625} T_{3}\right),
\end{aligned}
$$

где

$$
\dot{T}:=5 q \frac{\mathrm{d} T}{\mathrm{~d} q} .
$$

На самом деле, им был доказан следующий результат [99]. 
Теорема 8. Решение вышеуказанной системы в виде формалъного степенного ряда

$$
T_{j}=\sum_{n=0}^{\infty} t_{j, n} q^{n}, \quad j=0,1, \ldots, 6,
$$

с начальными условиями

$$
t_{0,0}=\frac{1}{5}, \quad t_{0,1}=24, \quad t_{4,0}=0, \quad t_{5,0}=-\frac{1}{3125}
$$

единственно. Более того, величина

$$
-\frac{\left(T_{4}-T_{0}^{5}\right)^{2}}{625 T_{5}^{3}}=5+2875 \frac{q}{1-q}+609250 \frac{2^{2} q^{2}}{1-q^{2}}+\cdots+N_{d} \frac{d^{3} q^{d}}{1-q^{d}}+\cdots
$$

представляет собой спаривание Юкавы семейства (74), а функиии (78) алгебраически независимы над $\mathbb{C}$ (на самом деле, над $\mathbb{C}(q, \log q)$, как показано в [161]).

При доказательстве алгебраической независимости в [161] и [99] рассматриваемые функции связываются с фундаментальным решением линейного дифференциального уравнения (75) и используется структура монодромии последнего. Вопрос о структуре монодромии рассматривался в ряде работ по этой тематике. Известно [26], что замыкание в топологии Зариского проективной группы монодромии (75), а также других гипергеометрических дифференциальных уравнений Калаби-Яу есть $\mathrm{Sp}_{4}(\mathbb{C})$ (это следует из (72)). Как можно выбрать “хороший” базис решений, в котором матрицы монодромии лежат в $\mathrm{Sp}_{4}(\mathbb{C})$ ? Возможный подход к построению такого базиса дан в [41] для всех арифметических гипергеометрических дифференциальных уравнений. K примеру, в [41] показано, что в качестве матриц монодромии в окрестности особых точек $z=0$ и $z=1 / 3125$ уравнения (75) можно взять симплектические матрицы

$$
\left(\begin{array}{cccc}
1 & 1 & 0 & 0 \\
0 & 1 & 0 & 0 \\
5 & 5 & 1 & 0 \\
0 & -5 & -1 & 1
\end{array}\right) \quad \text { и }\left(\begin{array}{llll}
1 & 0 & 0 & 0 \\
0 & 1 & 0 & 1 \\
0 & 0 & 1 & 0 \\
0 & 0 & 0 & 1
\end{array}\right)
$$

Отметим, что порождаемая матрицами (79) группа содержится в конгруэнцподгруппе

$$
\left\{\gamma \in \operatorname{Sp}_{4}(\mathbb{Z}): \gamma \equiv\left(\begin{array}{cccc}
1 & * & * & * \\
0 & 1 & * & * \\
0 & 0 & 1 & 0 \\
0 & 0 & * & 1
\end{array}\right) \quad(\bmod 5)\right\}
$$

конечного индекса в $\mathrm{Sp}_{4}(\mathbb{Z})$. Численно было установлено, что схожие конгруэнц-подгруппы конечного индекса в $\mathrm{Sp}_{4}(\mathbb{Z})$ содержат группы монодромии других дифференциальных уравнений Калаби-Яу. Исходя из этого наблюдения, возникает вопрос о том, можно ли связать возникающие в зеркальной симметрии функции типа (78) с модулярными формами Зигеля второй степени или, более обще, с неголоморфными модулярными формами второй степени. В следующем параграфе мы приводим некоторые частные результаты в этом направлении, обсуждавшиеся в [151]. 
2.4. $\mathrm{Sp}_{4}$-модулярность. Рассмотрим дифференциальное уравнение Калаби-Яу четвертого порядка. Его проективная группа монодромии $\Gamma \subset \operatorname{Sp}_{4}(\mathbb{R})$ соизмерима с дискретной подгруппой группы $\mathrm{Sp}_{4}(\mathbb{Z})$ (не обязательно конечного индекса). Как следствие, можно сформировать матрицу фундаментального решения

$$
\left(\begin{array}{cccc}
u_{3} & u_{3}^{\prime} & u_{3}^{\prime \prime} & u_{3}^{\prime \prime \prime} \\
u_{2} & u_{2}^{\prime} & u_{2}^{\prime \prime} & u_{2}^{\prime \prime \prime} \\
u_{1} & u_{1}^{\prime} & u_{1}^{\prime \prime} & u_{1}^{\prime \prime \prime} \\
u_{0} & u_{0}^{\prime} & u_{0}^{\prime \prime} & u_{0}^{\prime \prime \prime}
\end{array}\right)
$$

таким образом, что базис $u_{0}, u_{1}, u_{2}, u_{3}$ удовлетворяет уравнению

$$
W\left(u_{0}, u_{2}\right)+W\left(u_{1}, u_{3}\right)=0,
$$

а матрицы монодромии лежат в $\Gamma$; обозначение $W\left(u_{0}, u_{1}\right):=u_{0} u_{1}^{\prime}-u_{0}^{\prime} u_{1}$ используется для вронскиана. Определим функции

$$
w_{j l}=C z W\left(u_{j}, u_{l}\right)=C z\left(u_{j} u_{l}^{\prime}-u_{j}^{\prime} u_{l}\right), \quad w_{j l}=-w_{l j}, \quad 0 \leqslant j, l \leqslant 3,
$$

где $C \neq 0$ - некоторая нормировочная постоянная. Из (80) следует линейное соотношение $w_{02}+w_{13}=0$; также имеет место квадратичное соотношение

$$
w_{01} w_{23}+w_{02} w_{13}+w_{03} w_{12}=0,
$$

являющееся тавтологией в терминах $u_{j}$. Пять линейно независимых функций

$$
w_{01}, \quad w_{02}=-w_{13}, \quad w_{03}, \quad w_{12}, \quad w_{23}
$$

образуют решение линейного дифференциального уравнения пятого порядка (так называемого антисимметричного квадрата) с группой монодромии, сопряженной подгруппе, соизмеримой с дискретной подгруппой $O_{5}(\mathbb{Z}) \simeq \operatorname{Sp}_{4}(\mathbb{Z})$. Так возникает соответствие между дифференциальными уравнениями Калаби-Яу четвертого и пятого порядка.

Если теперь определить функции

$$
\tau_{1}(z):=\frac{w_{03}}{w_{01}}, \quad \tau_{2}(z):=\frac{w_{02}}{w_{01}}=\frac{-w_{13}}{w_{01}}, \quad \tau_{3}(z):=\frac{-w_{12}}{w_{01}}
$$

и собрать их в симметричную матрицу

$$
\mathrm{T}:=\left(\begin{array}{cc}
\tau_{1} & \tau_{2} \\
\tau_{2} & \tau_{3}
\end{array}\right), \quad \operatorname{det} \mathrm{T}=\frac{w_{23}}{w_{01}},
$$

то несложная проверка показывает, что матрицы монодромии $\gamma \in \Gamma$ задают традиционное $\mathrm{Sp}_{4}$-действие на $\mathrm{T}$ :

$$
\gamma: \mathrm{T} \mapsto(A \mathrm{~T}+B)(C \mathrm{~T}+D)^{-1}=\gamma \mathrm{T} \quad \text { для } \quad \gamma=\left(\begin{array}{cc}
A & B \\
C & D
\end{array}\right) \in \Gamma .
$$

Здесь $A, B, C, D-(2 \times 2)$-компоненты $(4 \times 4)$-матрицы $\gamma$ из $\operatorname{Sp}_{4}(\mathbb{R})$. Отметим, что из дифференциальной теории Галуа [161] следует алгебраическая независимость над $\mathbb{C}(z)$ трех компонент (81) матрицы $\mathrm{T}=\mathrm{T}(z)$. 
Значения многозначной функции $\tau:=\tau_{1}(z)$ лежат в некоторой области $H \subset \mathbb{C}$. Рассматривая $\mathrm{T}$ как матричнозначную функцию аргумента $\tau$, мы говорим, что функция $f(\mathrm{~T}(\tau)): H \rightarrow \mathbb{C}$ есть Г-модулярная форма веса $k$, если

$$
f(\gamma \mathrm{T})=\operatorname{det}(C \mathrm{~T}+D)^{k} \cdot f(\mathrm{~T}) \quad \text { при всех } \quad \gamma=\left(\begin{array}{cc}
A & B \\
C & D
\end{array}\right) \in \Gamma .
$$

Основанием для данного определения служит наблюдение [151], что обращение $z=z(\mathrm{~T}(\tau))$ отображения $\tau=\tau_{1}(z)$ в $(81),(82)$ является Г-модулярной формой нулевого веса, в то время как функция $w_{01}$, рассматриваемая как функция $\mathrm{T}=\mathrm{T}(\tau)$, является Г-модулярной формой веса 1. Заметим, что для производной по $z$ зеркального отображения $t(z)=u_{1}(z) / u_{0}(z)$ исходного дифференциального уравнения Калаби-Яу четвертого порядка и также для производной функции $\tau(z)$, представляющей собой зеркальное отображение получающегося дифференциального уравнения Калаби-Яу пятого порядка, можно выписать простые формулы

$$
\frac{\mathrm{d} t}{\mathrm{~d} z}=\frac{w_{01}}{C u_{0}^{2}}, \quad \frac{\mathrm{d} \tau_{1}}{\mathrm{~d} z}=\frac{u_{0}^{2}}{C g_{0} w_{01}^{2}},
$$

выражающие их через аналитические решения $u_{0}$ и $w_{01}$ уравнений. $\mathrm{B}$ свою очередь, из данных соотношений следует, что

$$
\tau_{1}(\tau)=\tau, \quad \tau_{2}(\tau)=-\int_{0}^{\tau} t(\tau) \mathrm{d} \tau, \quad \tau_{3}(\tau)=\int_{0}^{\tau} t(\tau)^{2} \mathrm{~d} \tau
$$

в случае, когда Т рассматривается как функция $\tau$.

Неприятное обстоятельство, связанное с определенной выше $\mathrm{Sp}_{4}$-модулярностью, заключается в том, что мнимая часть матрицы Т имеет неопределенную сигнатуру, в частности, Т не лежит в верхнем полупространстве Зигеля. Это было отмечено из геометрических соображений в [1]. Вместо этого М. Аганагич, В. Бушар и А. Клемм [1] предложили рассматривать неголоморфное вложение

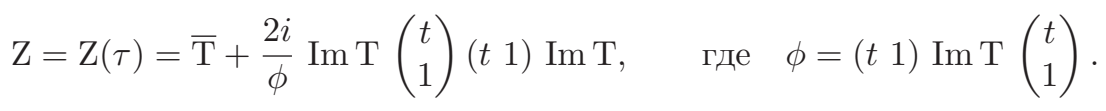

Тогда $\mathrm{Z}(\tau) \in \mathbb{H}_{2}$ при всех $\tau$, причем

$$
\gamma: \mathrm{Z} \mapsto(A \mathrm{Z}+B)(C \mathrm{Z}+D)^{-1}=\gamma \mathrm{Z} \quad \text { для } \quad \gamma=\left(\begin{array}{ll}
A & B \\
C & D
\end{array}\right) \in \Gamma ;
$$

дополнительно к этому, функция $w=\bar{\phi} \cdot w_{0}$ удовлетворяет соотношениям

$$
\gamma: w \mapsto \operatorname{det}(C \overline{\mathrm{Z}}+D) \cdot w
$$

Существуют по крайней мере два подхода к построению неголоморфных модулярных форм $w(\mathrm{Z})$, определенных на $\mathbb{H}_{2}$ и удовлетворяющих (85) для $\gamma$ из полной модулярной группы $\mathrm{Sp}_{4}(\mathbb{Z})$. Для этого можно использовать тета-ряды, соответствующие неопределенным квадратичным формам [114], или ряды Эйзенштейна [91; гл. 18]. Ниже на примере тривиального спаривания Юкавы 
и действия подгруппы конечного индекса $\Gamma^{\prime} \subset \mathrm{PSL}_{2}(\mathbb{Z})$ на $\tau=x+i y \in \mathbb{H}_{1}$ (т.е. $y>0)$ мы указываем возможный подход при обратном переводе построенных таким образом подходящих неголоморфных форм в объекты на одномерной области определения. Этот случай соответствует равенству $t=\tau$ двух зеркальных отображений.

При $t=\tau$ из (84) следует, что

$$
\mathrm{T}(\tau)=\left(\begin{array}{cc}
\tau & -\frac{1}{2} \tau^{2} \\
-\frac{1}{2} \tau^{2} & \frac{1}{3} \tau^{3}
\end{array}\right), \quad \mathrm{Z}(x+i y)=\left(\begin{array}{cc}
x & -\frac{1}{2} x^{2} \\
-\frac{1}{2} x^{2} & \frac{1}{3} x^{3}
\end{array}\right)+\frac{i}{2}\left(\begin{array}{cc}
y & -x y \\
-x y & x^{2} y+\frac{1}{3} y^{3}
\end{array}\right),
$$

поскольку $\phi=-\frac{4}{3} y^{3}$. При этом

$$
\operatorname{det} \mathrm{Z}=\frac{1}{12} \tau^{2}|\tau|^{2} \quad \text { и } \quad \operatorname{det} \operatorname{Im} \mathrm{Z}=\frac{1}{12} y^{4}=\frac{1}{12}(\operatorname{Im} \tau)^{4} .
$$

Несколько отличающееся от данного вложение было независимо получено Д. Загиром [153]. Соответствующее вложение группы $\mathrm{SL}_{2}(\mathbb{R})$ в $\mathrm{Sp}_{4}(\mathbb{R})$, именно

$$
\iota:\left(\begin{array}{ll}
a & b \\
c & d
\end{array}\right) \mapsto\left(\begin{array}{cccc}
a^{2} d+2 a b c & -3 a^{2} c & a b d+\frac{1}{2} b^{2} c & \frac{1}{2} b^{2} d \\
-a^{2} b & a^{3} & -\frac{1}{2} a b^{2} & -\frac{1}{6} b^{3} \\
4 a c d+2 b c^{2} & -6 a c^{2} & a d^{2}+2 b c d & b d^{2} \\
6 c^{2} d & -6 c^{3} & 3 c d^{2} & d^{3}
\end{array}\right)
$$

показывает, что при определенных арифметических условиях на $\Gamma^{\prime}$ (к примеру, $b \equiv 0(\bmod 6)) \Gamma^{\prime}$ отображается в подгруппу группы $\operatorname{Sp}_{4}(\mathbb{Z})$. Если мы теперь рассмотрим произвольную $\Gamma^{\prime}$-модулярную форму $w_{0}(\tau)$ веса 4 , то соответствующая функция $w(x+i y)=-\frac{4}{3} y^{3} \cdot w_{0}(x+i y)$ удовлетворяет на кривой

$$
\left\{\mathrm{Z}(\tau): \tau \in \mathbb{H}_{1}\right\}=\left\{\left(\begin{array}{cc}
x+\frac{i}{2} y & -\frac{1}{2} x^{2}-\frac{i}{2} x y \\
-\frac{1}{2} x^{2}-\frac{i}{2} x y & \frac{1}{3} x^{3}+\frac{i}{2} x^{2} y+\frac{i}{6} y^{3}
\end{array}\right): y>0\right\} \subset \mathbb{H}_{2}
$$

соотношениям (85). Таким образом, если мы изначально возьмем функцию $w(\mathrm{Z})$, удовлетворяющую закону преобразования (85), то остается лишь технический вопрос определить условия, при которых определенное на $\mathbb{H}_{1}$ обратное отображение $w_{0}(\tau)=-\frac{3}{4} w(\mathrm{Z}(\tau)) /(\operatorname{Im} \tau)^{3}$ является голоморфным (или мероморфным) в $\mathbb{H}_{1}$.

В общем случае $t \neq \tau$ картина, по-видимому, является более деликатной, из-за наличия трансцендентного соотношения между $\tau$ и $t$. Тем не менее, знание явных разложений в степенные ряды для $\tau$ и $t$ оставляет надежду на получение соответствующих обратных отображений и в таких ситуациях.

2.5. Формулы рамануджанова типа для $1 / \pi^{2}$. В настоящее время уже практически нет ничего загадочного в классических формулах Рамануджана для представления $1 / \pi$, примеры которых - формулы (4)-(6) и их многочисленные обобщения. По этому вопросу мы рекомендуем читателю ознакомиться с монографией [30] и недавно опубликованными обзорами [16], [181]. В отношении данных формул примечательным является не только явное присутствие гипергеометрического ряда в левой части, но также наличие чисто гипергеометрического аппарата [51], [65], [67], позволяющего доказывать некоmорые из таких представлений. В роли этого инструмента выступает теория 
Уилфа-Цайльбергера (WZ-теория) [105], [149] имеющая в своем арсенале алгоритм созидательного телескопирования. Даже несмотря на то, что такой подход не позволяет покрыть все многообразие представлений для $1 / \pi$ (хотя алгебраические преобразования, схожие с рассмотренными в теореме 7 , существенно расширяют применимость данного подхода), метод не перестает радовать неожиданными приятными сюрпризами. Х. Гиллера [65]-[68] смог применить WZ-теорию для доказательств новых обобщений рядов рамануджанова типа; именно,

$$
\begin{aligned}
\sum_{n=0}^{\infty} \frac{\left(\frac{1}{2}\right)_{n}^{5}}{n !^{5}}\left(20 n^{2}+8 n+1\right) \frac{(-1)^{n}}{2^{2 n}} & =\frac{8}{\pi^{2}}, \\
\sum_{n=0}^{\infty} \frac{\left(\frac{1}{2}\right)_{n}^{5}}{n !^{5}}\left(820 n^{2}+180 n+13\right) \frac{(-1)^{n}}{2^{10 n}} & =\frac{128}{\pi^{2}}, \\
\sum_{n=0}^{\infty} \frac{\left(\frac{1}{2}\right)_{n}^{3}\left(\frac{1}{4}\right)_{n}\left(\frac{3}{4}\right)_{n}}{n !^{5}}\left(120 n^{2}+34 n+3\right) \frac{1}{2^{4 n}} & =\frac{32}{\pi^{2}}, \\
\sum_{n=0}^{\infty} \frac{\left(\frac{1}{2}\right)_{n}^{3}\left(\frac{1}{3}\right)_{n}\left(\frac{2}{3}\right)_{n}}{n !^{5}}\left(74 n^{2}+27 n+3\right) \frac{3^{3 n}}{4^{3 n}} & =\frac{48}{\pi^{2}} .
\end{aligned}
$$

Более того, рассмотрение данного нового вида представлений для $1 / \pi^{2}$ подтолкнуло Гиллеру [66] и Гиллеру с Алмквистом [2] к численному открытию дополнительных семи представлений:

$$
\begin{aligned}
& \sum_{n=0}^{\infty} \frac{\left(\frac{1}{2}\right)_{n}\left(\frac{1}{4}\right)_{n}\left(\frac{3}{4}\right)_{n}\left(\frac{1}{6}\right)_{n}\left(\frac{5}{6}\right)_{n}}{n !^{5}}\left(1640 n^{2}+278 n+15\right) \frac{(-1)^{n}}{2^{10 n}} \stackrel{?}{=} \frac{256 \sqrt{3}}{3 \pi^{2}} \\
& \sum_{n=0}^{\infty} \frac{\left(\frac{1}{2}\right)_{n}\left(\frac{1}{4}\right)_{n}\left(\frac{3}{4}\right)_{n}\left(\frac{1}{3}\right)_{n}\left(\frac{2}{3}\right)_{n}}{n !^{5}}\left(252 n^{2}+63 n+5\right) \frac{(-1)^{n}}{48^{n}} \stackrel{?}{=} \frac{48}{\pi^{2}}, \\
& \sum_{n=0}^{\infty} \frac{\left(\frac{1}{2}\right)_{n}\left(\frac{1}{3}\right)_{n}\left(\frac{2}{3}\right)_{n}\left(\frac{1}{6}\right)_{n}\left(\frac{5}{6}\right)_{n}}{n !^{5}}\left(5418 n^{2}+693 n+29\right) \frac{(-1)^{n}}{80^{3 n}} \stackrel{?}{=} \frac{128 \sqrt{5}}{\pi^{2}}, \\
& \sum_{n=0}^{\infty} \frac{\left(\frac{1}{2}\right)_{n}\left(\frac{1}{3}\right)_{n}\left(\frac{2}{3}\right)_{n}\left(\frac{1}{6}\right)_{n}\left(\frac{5}{6}\right)_{n}}{n !^{5}}\left(1930 n^{2}+549 n+45\right) \frac{(-1)^{n} 3^{6 n}}{2^{12 n}} \stackrel{?}{=} \frac{384}{\pi^{2}} \\
& \sum_{n=0}^{\infty} \frac{\left(\frac{1}{2}\right)_{n}\left(\frac{1}{3}\right)_{n}\left(\frac{2}{3}\right)_{n}\left(\frac{1}{6}\right)_{n}\left(\frac{5}{6}\right)_{n}}{n !^{5}}\left(532 n^{2}+126 n+9\right) \frac{3^{6 n}}{5^{6 n}} \stackrel{?}{=} \frac{375}{4 \pi^{2}} \\
& \sum_{n=0}^{\infty} \frac{\left(\frac{1}{2}\right)_{n}\left(\frac{1}{8}\right)_{n}\left(\frac{3}{8}\right)_{n}\left(\frac{5}{8}\right)_{n}\left(\frac{7}{8}\right)_{n}}{n !^{5}}\left(1920 n^{2}+304 n+15\right) \frac{1}{7^{4 n}} \stackrel{?}{=} \frac{56 \sqrt{7}}{\pi^{2}}, \\
& \sum_{n=0}^{\infty} \frac{\left(\frac{1}{2}\right)_{n}^{3}\left(\frac{1}{3}\right)_{n}\left(\frac{2}{3}\right)_{n}}{n !^{5}}\left((32-216 \phi) n^{2}+(18-162 \phi) n+(3-30 \phi)\right)(3 \phi)^{3 n} \stackrel{?}{=} \frac{3}{\pi^{2}},
\end{aligned}
$$


где

$$
\phi=\left(\frac{\sqrt{5}-1}{2}\right)^{5}=0.09016994 \ldots .
$$

Заметим, что эта постоянная появляется в асимптотике приближений Апери к $\zeta(2)($ ср. с $(28)$ и $(29)$ в $§ 1.1)$.

Обратим также внимание на "3D" тождество

$$
\sum_{n=0}^{\infty} \frac{\left(\frac{1}{2}\right)_{n}^{7}}{n !^{7}}\left(168 n^{3}+76 n^{2}+14 n+1\right) \frac{1}{2^{6 n}} \stackrel{?}{=} \frac{32}{\pi^{3}},
$$

открытое Б. Гуревичем в 2002 г. (с использованием алгоритма целочисленных отношений), и на последнее достижение в этой области - представление

$$
\sum_{n=0}^{\infty} \frac{\left(\frac{1}{2}\right)_{n}^{7}\left(\frac{1}{4}\right)_{n}\left(\frac{3}{4}\right)_{n}}{n !^{9} 2^{12 n}}\left(43680 n^{4}+20632 n^{3}+4340 n^{2}+466 n+21\right) \stackrel{?}{=} \frac{2048}{\pi^{4}},
$$

полученное Дж. Кулленом [44].

В работах [2], [69] и [151] исследуется взаимосвязь тождеств (86)-(96) с рассмотренными в $§ 2.2$ дифференциальными уравнениями Калаби-Яу. Стандартным примером, связанным с формулами Гиллеры (86) и (87), является гипергеометрический ряд

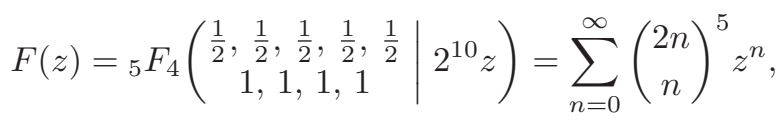

удовлетворяющий линейному дифференциальному уравнению пятого порядка

$$
\left(\theta^{5}-32 z(2 \theta+1)^{5}\right) Y=0, \quad \text { где } \quad \theta=z \frac{\mathrm{d}}{\mathrm{d} z} .
$$

Если $G(z)$ - другое решение последнего уравнения вида $F(z) \log z+F_{1}(z)$, где $F_{1}(z) \in z \mathbb{Q}[[z]]$, то аналитическая в нуле функция

$$
\widetilde{F}(z)=\left(1-2^{10} z\right)^{-1 / 2} \operatorname{det}\left(\begin{array}{cc}
F & G \\
\theta F & \theta G
\end{array}\right)^{1 / 2}
$$

удовлетворяет уравнению четвертого порядка

$$
\left(\theta^{4}-16 z\left(128 \theta^{4}+256 \theta^{3}+304 \theta^{2}+176 \theta+39\right)+2^{20} z^{2}(\theta+1)^{4}\right) Y=0,
$$

которое входит в таблицу из [3] под номером 204. Квадратичное преобразование [5]

$$
\frac{1+z}{(1-z)^{2}} \widetilde{F}\left(\frac{-z}{(1-z)^{2}}\right)=\sum_{n=0}^{\infty}\left(\sum_{k=0}^{n} 4^{n-k}\left(\begin{array}{c}
2 k \\
k
\end{array}\right)^{2}\left(\begin{array}{c}
2 n-2 k \\
n-k
\end{array}\right)\right)^{2} z^{n}
$$

дает явное выражение новой функции $\widetilde{F}(z)$ в терминах адамарова квадрата ряда

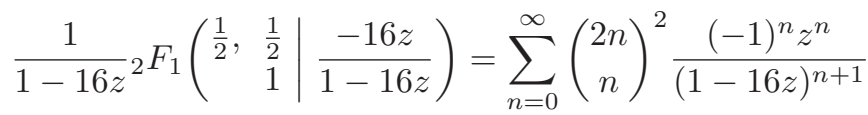

$$
\begin{aligned}
& =\sum_{n=0}^{\infty}\left(\sum_{k=0}^{n} 4^{n-k}\left(\begin{array}{c}
2 k \\
k
\end{array}\right)^{2}\left(\begin{array}{c}
2 n-2 k \\
n-k
\end{array}\right)\right) z^{n},
\end{aligned}
$$


допускающего модулярную параметризацию. Как уравнение четвертого порядка (97), так и дифференциальное уравнение (четвертого порядка) для правой части (98) являются уравнениями типа Калаби-Яу. Эти дифференциальные уравнения имеют первостепенное значение при численном открытии многих представлений для $1 / \pi^{2}$; детали этого могут быть почерпнуты из [2].

Более “тонкий” результат применения подхода Уилфа-Цайльбергера состоит в получении функциональных тождеств, включающих в качестве частных случаев числовые представления. Ожидаемое преимущество функциональных примеров состоит в том, что они могут быть доказаны "проще" благодаря использованию дополнительных функциональных (например, дифференциальных) уравнений. Известно несколько индивидуальных методов перехода от числовых представлений к функциональным, из которых многие основываются на хитроумном применении алгоритма Госпера-Цайльбергера созидательного телескопирования [105].

В [67] даны примеры, относящиеся к ряду Рамануджана (4) и ряду Гиллеры (86). Рассмотрим

$$
\begin{aligned}
\sum_{n=0}^{\infty} & \frac{\left(a+\frac{1}{2}\right)_{n}^{3}}{(a+1)_{n}^{3}}(6(n+a)+1) \cdot \frac{1}{4^{n}} \\
& =\frac{4^{a+1}}{\pi \cos ^{2} \pi a} \cdot\left(\frac{\Gamma(a+1) \Gamma\left(\frac{1}{2}\right)}{\Gamma\left(a+\frac{1}{2}\right)}\right)^{3}+\frac{(4 a)^{2}}{2 a-1} \sum_{n=0}^{\infty} \frac{\left(\frac{1}{2}\right)_{n}\left(a+\frac{1}{2}\right)_{n}}{(a+1)_{n}\left(\frac{3}{2}-a\right)_{n}}
\end{aligned}
$$

и обозначим через $f(a)$ любую из частей этого равенства. Тогда

$$
\begin{aligned}
& \sum_{n=0}^{\infty} \frac{\left(a+\frac{1}{2}\right)_{n}^{5}}{(a+1)_{n}^{5}}\left(20(n+a)^{2}+8(n+a)+1\right) \cdot \frac{(-1)^{n}}{4^{n}} \\
& \quad=\frac{2}{\pi \cos \pi a} \cdot\left(\frac{\Gamma(a+1) \Gamma\left(\frac{1}{2}\right)}{\Gamma\left(a+\frac{1}{2}\right)}\right)^{2} f(a)+\frac{2^{5} a^{3}}{2 a-1} \sum_{n=0}^{\infty} \frac{\left(\frac{1}{2}\right)_{n}^{2}\left(a+\frac{1}{2}\right)_{n}}{(a+1)_{n}^{2}\left(\frac{3}{2}-a\right)_{n}} .
\end{aligned}
$$

Специализация $a=0$ в (99) и (100) приводит соответственно к числовым тождествам (4) и (86).

Еще одно яркое арифметическое свойство, скрытое в формулах Рамануджана для $1 / \pi$ и их обобщениях, основано на так называемых суперсравнениях. Оказывается, что при обрывании соответствующего гипергеометрического ряда на члене с номером $n=p-1$ всегда получаются сравнения по модулю высших степеней простого числа $p>3$, не делящего знаменатель аргумента. Так, например,

$$
\begin{gathered}
\sum_{n=0}^{p-1} \frac{\left(\frac{1}{2}\right)_{n}\left(\frac{1}{4}\right)_{n}\left(\frac{3}{4}\right)_{n}}{n !^{3}}(20 n+3) \frac{(-1)^{n}}{2^{2 n}} \equiv 3\left(\frac{-1}{p}\right) p \quad\left(\bmod p^{3}\right), \\
\sum_{n=0}^{p-1} \frac{\left(\frac{1}{2}\right)_{n}^{3}\left(\frac{1}{4}\right)_{n}\left(\frac{3}{4}\right)_{n}}{n !^{5}}\left(120 n^{2}+34 n+3\right) \frac{1}{2^{4 n}} \equiv 3 p^{2} \quad\left(\bmod p^{5}\right), \\
\sum_{n=0}^{p-1} \frac{\left(\frac{1}{2}\right)_{n}^{7}\left(\frac{1}{4}\right)_{n}\left(\frac{3}{4}\right)_{n}}{n !^{9} 2^{12 n}}\left(43680 n^{4}+20632 n^{3}+4340 n^{2}+466 n+21\right) \stackrel{?}{\equiv} 21 p^{4} \quad\left(\bmod p^{9}\right) .
\end{gathered}
$$


Имеющиеся доказательства [71], [137], [183] снова опираются на теорию УилфаЦайльбергера. В работе [70] обсуждается общий вид подобных суперсравнений, включая конечные аналоги "иррациональных" тождеств (96) рамануджанова типа.

\section{3. Суммы по решеткам и меры Малера}

3.1. L-ряды Дирихле и меры Малера. Мера Малера $M(P):=e^{\mathrm{m}(P)}$ многочлена Лорана $P\left(x_{1}, \ldots, x_{n}\right)$, где $\mathrm{m}(P)$ определено в $(9)$, есть среднее геометрическое значения $|P|$ на торе

$$
\mathbb{T}^{n}=\left\{\left(x_{1}, \ldots, x_{n}\right) \in \mathbb{C}^{n}:\left|x_{1}\right|=\cdots=\left|x_{n}\right|=1\right\} .
$$

Первоначальное определение Малера [93] относится к случаю $n=1$, в котором $\mathrm{m}(P)$ может быть также выражена в виде

$$
\mathrm{m}(P)=\log \left|a_{0}\right|+\sum_{j=1}^{d} \max \left\{0, \log \left|\alpha_{j}\right|\right\}
$$

для многочлена $P(x)=a_{0} \prod_{j=1}^{d}\left(x-\alpha_{j}\right)$. Это следует из классической формулы Йенсена. Для многочленов $P(x)$ с целыми коэффициентами несложно проверить, что $\mathrm{m}(P) \geqslant 0$, причем равенство $\mathrm{m}(P)=0$ достигается только на многочленах $P$ со старшим коэффициентом $1\left(a_{0}=1\right)$, все нули которых лежат внутри единичного диска (вследствие теоремы Кронекера такой многочлен является произведением монома $x^{a}$ и многочлена деления круга). Уже в 1933 г. Д. Лемер [88] поставил вопрос о том, можно ли сделать $\mathrm{m}(P)$ сколь угодно малым, но положительным при условии $P(x) \in \mathbb{Z}[x]$. Наименьшее полученное Лемером значение составляет

$\mathrm{m}\left(x^{10}+x^{9}-x^{7}-x^{6}-x^{5}-x^{4}-x^{3}+x+1\right)=\log (1.17628081 \ldots)=0.16235761 \ldots ;$

оно до сих пор не улучшено, несмотря на обширные вычисления, проведенные Д. Бойдом, М. Моссингофом и другими. Хотя вопрос Лемера лежит в совершенно иной плоскости при изучении мер Малера, он явился побудительным мотивом введения данного выше определения $\mathrm{m}(P)$ в случае многих переменных из-за наличия следующей предельной формулы, доказанной Бойдом в 1981 г. в работе [33]:

$$
\mathrm{m}\left(P\left(x, x^{N}\right)\right) \rightarrow \mathrm{m}(P(x, y)) \quad \text { при } N \rightarrow \infty .
$$

Лишь в 1981 г. пришло понимание того, что мера Малера от $n$ переменных может иметь “геометрические" корни. Именно тогда К. Смит представил элегантную формулу [33]

$$
\mathrm{m}(1+x+y)=\frac{3 \sqrt{3}}{4 \pi} L\left(\chi_{-3}, 2\right)=L^{\prime}\left(\chi_{-3},-1\right),
$$

где

$$
L\left(\chi_{-3}, s\right)=\sum_{n=1}^{\infty} \frac{\chi_{-3}(n)}{n^{s}}=1-\frac{1}{2^{s}}+\frac{1}{4^{s}}-\frac{1}{5^{s}}+\cdots
$$


- $L$-функция, отвечающая вещественному нечетному характеру Дирихле по модулю 3.

Доказательство формулы Смита сто́ит упоминания здесь. Так как $1+x+y$ есть линейная функция от $y$, применение формулы Йенсена к одному из интегралов в (9) дает

$\mathrm{m}(1+x+y)=\mathrm{m}(1-x+y)=\frac{1}{2 \pi} \int_{0}^{2 \pi} \log ^{+}\left|e^{i t}-1\right| \mathrm{d} t=\frac{1}{2 \pi} \int_{0}^{2 \pi / 3} \log \left|e^{i t}-1\right| \mathrm{d} t$,

где $\log ^{+} x=\max \{0, \log x\}$. Таким образом, $\mathrm{m}(1+x+y)$ дается специальным значением интеграла Клаузена

$$
\mathrm{Cl}_{2}(\theta)=-\int_{0}^{\theta} \log \left|e^{i t}-1\right| \mathrm{d} t=\sum_{k=1}^{\infty} \frac{\sin (k \theta)}{k^{2}},
$$

откуда и вытекает требуемый результат.

Похожий прием применим ко многим многочленам $P(x, y)=A(x) y+B(x)$, где $A(x)$ и $B(x)$ - многочлены деления круга и решения уравнения $|A(x)|=$ $|B(x)|$ на окружности $|x|=1$ являются корнями из единицы. К примеру [34],

$$
\mathrm{m}(1+x+y-x y)=\frac{2}{\pi} L\left(\chi_{-4}, 2\right)=L^{\prime}\left(\chi_{-4},-1\right),
$$

где $L\left(\chi_{-4}, 2\right)=G-$ постоянная Каталана (см. (13)),

$$
\begin{aligned}
\mathrm{m}\left(1+x+x^{2}+y\right) & =\frac{3}{2} L^{\prime}\left(\chi_{-4},-1\right), \\
\mathrm{m}\left(1+x+y+x^{2} y\right) & =\frac{3}{2} L^{\prime}\left(\chi_{-3},-1\right) .
\end{aligned}
$$

Позднее В. Майо и Ж. Касень [95] установили общую формулу для меры $\mathrm{m}\left(a_{0}+a_{1} x+a_{2} y\right)$ при произвольных комплексных $a_{j}$ в терминах дилогарифма Блоха-Вигнера

$$
\mathscr{D}(z)=\operatorname{Im}\left(\sum_{n=1}^{\infty} \frac{z^{n}}{n^{2}}+\log |z| \log (1-z)\right) .
$$

Если $\left|a_{0}\right|,\left|a_{1}\right|,\left|a_{2}\right|$ - длины сторон плоского треугольника, а $\alpha_{0}, \alpha_{1}, \alpha_{2}$ - углы при противолежащих вершинах, то

$$
\mathrm{m}\left(a_{0}+a_{1} x+a_{2} y\right)=\frac{\alpha_{0}}{\pi} \log \left|a_{0}\right|+\frac{\alpha_{1}}{\pi} \log \left|a_{1}\right|+\frac{\alpha_{2}}{\pi} \log \left|a_{2}\right|+\frac{1}{\pi} \mathscr{D}\left(\frac{\left|a_{1}\right|}{\left|a_{0}\right|} e^{i \alpha_{2}}\right) ;
$$

в противном случае

$$
\mathrm{m}\left(a_{0}+a_{1} x+a_{2} y\right)=\log \max \left\{\left|a_{0}\right|,\left|a_{1}\right|,\left|a_{2}\right|\right\} .
$$

Уже из этого результата видна тесная связь между вычислением мер Малера и $K$-теорией. По этой причине не кажется совершенно неожиданным тот факт, что более сложные многочлены $P(x, y)$ приводят к мерам Малера, выраженным через специальные значения $L$-функций эллиптических кривых. Здесь аналогом значения

$$
\frac{d^{3 / 2} L\left(\chi_{-d}, 2\right)}{4 \pi}=L^{\prime}\left(\chi_{-d},-1\right)
$$


является

$$
b_{E}=\frac{N L(E, 2)}{4 \pi^{2}}=L^{\prime}(E, 0),
$$

где $N$ - кондуктор эллиптической кривой $E$. При этом последнее равенство справедливо только в том случае, если $E$ - модулярная кривая (т.е. гладкая кубическая кривая над $\mathbb{Q}$, содержащая рациональную точку; отметим, что согласно гипотезе Шимуры-Таниямы, теперь теореме, все эллиптические кривые над $\mathbb{Q}$ являются модулярными). Другими словами, найдутся многочлены $P_{E}(x, y)$, для которых значение $\mathrm{m}\left(P_{E}\right) / b_{E}$ (предположительно) рационально. Без указания весомых $K$-теоретических причин существования таких формул мы лишь схематично поясним, почему это так, на упомянутом во введении примере Денингера (12).

Определим

$$
P(x, y)=1+x+\frac{1}{x}+y+\frac{1}{y} .
$$

Положим $x=e^{i t}$ и рассмотрим $P(x, y)$ как многочлен по $y$. Тогда

$$
|P(x, y)|=\left|1+y(1+2 \cos t)+y^{2}\right|=\left|\left(y-y_{1}(t)\right)\left(y-y_{2}(t)\right)\right|,
$$

где $y_{1}(t)=-b-\sqrt{b^{2}-1}$ и $b=b(t)=\frac{1}{2}+\cos t$. В соответствии с формулой Йенсена

$$
\mathrm{m}(P)=\frac{1}{\pi} \int_{0}^{\pi} \log ^{+}\left|y_{1}(t)\right| \mathrm{d} t .
$$

Поскольку произведение корней $y_{1}(t)$ и $y_{2}(t)$ равно 1 , то $\left|y_{1}(t)\right|>1>\left|y_{2}(t)\right|$ в точности, когда корни действительны и не равны между собой, т.е. когда $\cos t>\frac{1}{2}$ и, следовательно, $|t|<\frac{\pi}{3}$. Таким образом,

$$
\mathrm{m}(P)=\int_{0}^{\pi / 3} \log \left(b+\sqrt{b^{2}-1}\right) \mathrm{d} t .
$$

Этот интеграл может быть вычислен численно, хотя, конечно, существует множество других способов его представления. Например,

$$
\begin{aligned}
\mathrm{m}(P) & =\frac{1}{(2 \pi)^{2}} \int_{0}^{2 \pi} \int_{0}^{2 \pi} \log (1+2 \cos t+2 \cos s) \mathrm{d} t \mathrm{~d} t \\
& =\frac{1}{4} \cdot{ }_{3} F_{2}\left(\begin{array}{c}
\frac{1}{2}, \frac{1}{2}, \frac{1}{2} \\
1, \frac{3}{2}
\end{array} \mid \frac{1}{16}\right)=4 \sum_{k=0}^{\infty}\left(\begin{array}{c}
2 k \\
k
\end{array}\right)^{2} \frac{(1 / 16)^{2 k+1}}{2 k+1} ;
\end{aligned}
$$

этот результат был нами упомянут в (11). Аналогичным образом можно получить более общее гипергеометрическое представление [120], [86], [123]

$$
\mathrm{m}\left(\alpha+x+x^{-1}+y+y^{-1}\right)=\frac{\alpha}{4} \operatorname{Re}_{3} F_{2}\left(\begin{array}{c}
\frac{1}{2}, \frac{1}{2}, \frac{1}{2} \\
1,1
\end{array} \mid \frac{\alpha^{2}}{16}\right)
$$

при $\alpha>0$.

С другой стороны, с помощи когомологической интерпретации $\mathrm{m}(P(x, y))$, Денингер [47] получил представление этой меры Малера в виде ряда Эйзенштейна-Кронекера эллиптической кривой $E$ с кондуктором 15, заданной уравнением

$$
1+x+\frac{1}{x}+y+\frac{1}{y}=0 .
$$


В предположении гипотезы Бейлинсона этот ряд выражается в терминах $L$ функции Дирихле этой кривой, так что в итоге получается тождество

$$
\mathrm{m}(P)=r \frac{15}{(2 \pi)^{2}} L(E, 2)=r L^{\prime}(E, 0)
$$

где $r$ - рациональное число (не оговоренное в гипотезе Бейлинсона). Наконец, численно было проверено, что $r=1.00000000$. . (с точностью до 200 десятичных знаков), что дает основание предполагать (10) верным. Из теоремы о модулярности следует, что для $L$-функции $L(E, s)=\sum_{k=1}^{\infty} a_{k} k^{-s}$ эллиптической кривой $E$ с кондуктором $N$ функция $f(\tau)=\sum_{k=1}^{\infty} a_{k} q^{k}$, где $q=e^{2 \pi i \tau}$, является параболической формой относительно модулярной группы $\Gamma_{0}(N)$. В случае Денингера $N=15$ и

$$
f(\tau)=\sum_{k=1}^{\infty} a_{k} q^{k}=q \prod_{m=1}^{\infty}\left(1-q^{m}\right)\left(1-q^{3 m}\right)\left(1-q^{5 m}\right)\left(1-q^{15 m}\right) .
$$

Согласно пентагональной теореме Эйлера

$$
\eta(\tau)=q^{1 / 24} \prod_{m=1}^{\infty}\left(1-q^{m}\right)=\sum_{n \in \mathbb{Z}}(-1)^{n} q^{(6 n+1)^{2} / 24}
$$

и ввиду указанного выше гипергеометрического представления окончательная формула имеет вид (12).

Следует отметить, что несмотря на происхождение формулы (12) мера Малера в нее не входит: формула представляет собой (гипергеометрическое) представление четырехкратной суммы в терминах однократной.

\section{2. Четырехкратные суммы по решеткам. Определим}

$$
\begin{aligned}
F(a, b, c, d) & =(a+b+c+d)^{2} \\
\times & \sum_{\substack{n_{j}=-\infty \\
j=1,2,3,4}}^{\infty} \frac{(-1)^{n_{1}+n_{2}+n_{3}+n_{4}}}{\left(a\left(6 n_{1}+1\right)^{2}+b\left(6 n_{2}+1\right)^{2}+c\left(6 n_{3}+1\right)^{2}+d\left(6 n_{4}+1\right)^{2}\right)^{2}},
\end{aligned}
$$

где суммирование производится методом

$$
\sum_{\substack{n_{j}=-\infty \\ j=1,2,3,4}}^{\infty}=\lim _{M \rightarrow \infty} \sum_{n_{1}=-M}^{M} \cdots \sum_{n_{4}=-M}^{M}
$$

Также положим

$$
F(b, c)=F(1, b, c, b c) .
$$

Известно много случаев, в которых $F(a, b, c, d)$ можно упростить (иногда только гипотетически) до однократной суммы. Например, для (12) имеем

$$
F(3,5)=\frac{\pi^{2}}{15} \cdot{ }_{3} F_{2}\left(\begin{array}{c}
\frac{1}{2}, \frac{1}{2}, \frac{1}{2} \\
1, \frac{3}{2}
\end{array} \mid \frac{1}{16}\right) .
$$


Другое представление, сформулированное Бойдом в качестве гипотезы и в настоящий момент доказанное в [124], связано с эллиптическими кривыми с кондуктором 20; оно эквивалентно соотношению

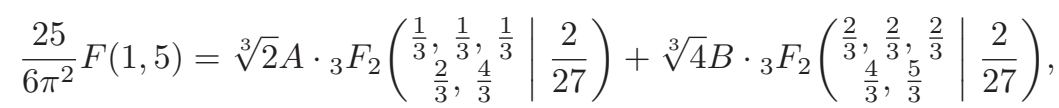

где

$$
A=\frac{\sqrt[3]{2} \Gamma\left(\frac{1}{6}\right) \Gamma\left(\frac{1}{3}\right) \Gamma\left(\frac{1}{2}\right)}{8 \sqrt{3} \pi^{2}} \quad \text { и } \quad B=\frac{\Gamma^{3}\left(\frac{2}{3}\right)}{16 \pi^{2}} .
$$

При рассмотрении более общих сумм по решеткам часто возникают гипергеометрические функции иррациональных аргументов. Так, например, в обозначении $\phi=(1+\sqrt{5}) / 2$ выполнено

$$
\frac{225}{32 \sqrt{5} \pi^{2}} F(1,5,5,5)=\frac{A}{\sqrt[3]{\phi}} \cdot{ }_{3} F_{2}\left(\begin{array}{c}
\frac{1}{3}, \frac{1}{3}, \frac{1}{3} \\
\frac{2}{3}, \frac{4}{3}
\end{array}\right)+\frac{\frac{1}{\phi}}{\sqrt[3]{\phi^{2}}} \cdot{ }_{3} F_{2}\left(\begin{array}{c}
\frac{2}{3}, \\
\frac{4}{3}, \frac{2}{3}, \frac{2}{3}
\end{array} \mid \frac{1}{\phi}\right) .
$$

Несмотря на то, что соотношения для $F(1,5,5,5)$ и $F(1,5)=F(1,1,5,5)$ кажутся частными случаями общих формул для $F(a, b, c, d)$, точная природа подобных представлений остается неясной.

Стандартный аналитический метод доказательства гипергеометрических представлений заключается в сведении рассматриваемой четырехкратной суммы по решетке к двойной, с последующей интерпретацией двойной суммы как специального значения эллиптической функции. К примеру,

$$
F(1,5,5,5)=16^{2} \sum_{\substack{n=-\infty \\ k=0}}^{\infty} \frac{(-1)^{n+k}(2 k+1)}{\left((6 n+1)^{2}+15(2 k+1)^{2}\right)^{2}} .
$$

Преобразования подобного рода являются следствиями известных результатов для $q$-рядов и встречаются крайне редко. Приведенная двойная сумма использует следствие тождества Якоби для тройного произведения:

$$
q^{2} \prod_{n=1}^{\infty}\left(1-q^{3 n}\right)\left(1-q^{15 n}\right)^{3}=\sum_{\substack{n=-\infty \\ k=0}}^{\infty}(-1)^{n+k}(2 k+1) q^{\left(15(2 k+1)^{2}+(6 n+1)^{2}\right) / 8}
$$

Отметим, что это уравнение дает пример лакунарной модулярной формы. Также отметим, что только в лакунарных случаях возможно сведение к двукратной сумме. Двойные суммы по решеткам можно в дальнейшем вычислить с помощью теорий Рамануджана эллиптических функций и модулярных уравнений [11], [19]. Многие такие вычисления весьма трудоемки.

В [96] приведен конечный список эллиптических кривых, для которых $L$ функции связаны с четырьмя эта-произведениями. Предположим, что $E_{N}-$ эллиптическая кривая с кондуктором $N$. Тогда $L\left(E_{N}, 2\right)=F(b, c)$ для приведенных в таблице 3 значений $N$ и $(b, c)$. Отметим, что при $N=11$ нет известного гипергеометрического представления, однако в этом случае можно получить выражение в терминах меры Малера, именно,

$$
\frac{77}{4 \pi^{2}} F(1,11)=\mathrm{m}((1+x)(1+y)(1+x+y)+x y) .
$$




\begin{tabular}{|c|c|}
\hline$N$ & $(b, c)$ \\
\hline 11 & $(1,11)$ \\
14 & $(2,7)$ \\
15 & $(3,5)$ \\
20 & $(1,5)$ \\
24 & $(2,3)$ \\
27 & $(1,3)$ \\
32 & $(1,2)$ \\
36 & $(1,1)$ \\
\hline
\end{tabular}

ТАБлицА 3. Соответствие $L\left(E_{N}, 2\right)=F(b, c)$ для эллиптических кривых $E_{N}$ с кондуктором $N$

Этот результат был выдвинут Бойдом в качестве гипотезы и недавно установлен Ф. Брюно [36] с использованием методов $K$-теории.

В заключение приведем список гипергеометрических представлений для соответствующих сумм по решеткам $F(b, c)=L\left(E_{N}, 2\right)$ :

$$
\begin{aligned}
& \frac{9}{2 \pi^{2}} F(1,1)=\frac{1}{9} \log 54-\frac{1}{81}{ }_{4} F_{3}\left(\begin{array}{c|c}
\frac{4}{3}, \frac{5}{3}, 1,1 & 1 \\
2,2,2 & \frac{1}{2}
\end{array}\right), \\
& \frac{16}{\pi^{2}} F(1,2)=2 \log 2+\frac{1}{8}{ }_{4} F_{3}\left(\begin{array}{c|c}
\frac{3}{2}, \frac{3}{2}, 1,1 & -\frac{1}{4} \\
2,2,2 & ,
\end{array}\right.
\end{aligned}
$$

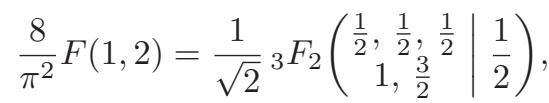

$$
\begin{aligned}
& \frac{81}{4 \pi^{2}} F(1,3)=\log 6+\frac{1}{108}{ }_{4} F_{3}\left(\begin{array}{c|c}
\frac{4}{3}, \frac{5}{3}, 1,1 & -\frac{1}{8} \\
2,2,2 & ,
\end{array}\right.
\end{aligned}
$$

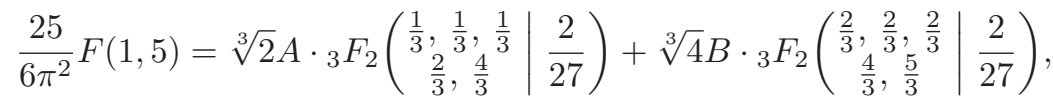

$$
\begin{aligned}
& \frac{40}{3 \pi^{2}} F(1,5)=\frac{5}{3} \log 2-\frac{1}{16}{ }_{4} F_{3}\left(\begin{array}{c|c}
\frac{4}{3}, \frac{5}{3}, 1,1 & \frac{27}{32} \\
2,2,2 & ,
\end{array}\right.
\end{aligned}
$$

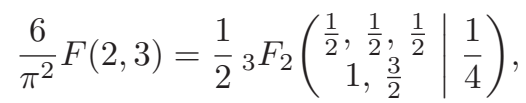

$$
\begin{aligned}
& \frac{24}{\pi^{2}} F(2,3)=3 \log 2-\frac{1}{32}{ }_{4} F_{3}\left(\begin{array}{c|c}
\frac{3}{2}, \frac{3}{2}, 1,1 & 1 \\
2,2,2 & \frac{1}{4}
\end{array}\right), \\
& \frac{15}{\pi^{2}} F(2,3)=\frac{1}{2} \log 18-\frac{1}{9}{ }_{4} F_{3}\left(\begin{array}{c|c}
\frac{3}{2}, \frac{3}{2}, 1,1 & \frac{8}{9} \\
2,2,2 & 9
\end{array}\right) \\
& \frac{9}{\pi^{2}} F(2,3)=\frac{1}{2} \log 2+{ }_{4} F_{3}\left(\begin{array}{c|c}
\frac{3}{2}, \frac{3}{2}, 1,1 \\
2,2,2
\end{array} \mid-8\right), \\
& \frac{14}{\pi^{2}} F(2,7)=A \cdot{ }_{3} F_{2}\left(\begin{array}{c}
\frac{1}{3}, \frac{1}{3}, \frac{1}{3} \\
\frac{2}{3}, \frac{4}{3}
\end{array} \mid-\frac{1}{27}\right)-B \cdot{ }_{3} F_{2}\left(\begin{array}{c}
\frac{2}{3}, \frac{2}{3}, \frac{2}{3} \\
\frac{4}{3}, \frac{5}{3}
\end{array} \mid-\frac{1}{27}\right), \\
& \frac{49}{2 \pi^{2}} F(2,7)=\log 5-\frac{2}{125}{ }_{4} F_{3}\left(\begin{array}{c|c}
\frac{4}{3}, \frac{5}{3}, 1,1 & \frac{27}{125} \\
2,2,2 & ,
\end{array}\right.
\end{aligned}
$$




$$
\begin{aligned}
& \frac{15}{4 \pi^{2}} F(3,5)=\frac{1}{4}{ }_{3} F_{2}\left(\begin{array}{c}
\frac{1}{2}, \frac{1}{2}, \frac{1}{2} \\
1, \frac{3}{2}
\end{array} \mid \frac{1}{16}\right), \\
& \frac{45}{2 \pi^{2}} F(3,5)=\log 5-\frac{2}{25}{ }_{4} F_{3}\left(\begin{array}{c|c}
\frac{3}{2}, \frac{3}{2}, 1,1 & \frac{16}{25} \\
2,2,2 & ,
\end{array}\right. \\
& \frac{165}{4 \pi^{2}} F(3,5)=4 \log 2-\frac{1}{128}{ }_{4} F_{3}\left(\begin{array}{c|c}
\frac{3}{2}, \frac{3}{2}, 1,1 & \frac{1}{16} \\
2,2,2 & ,
\end{array}\right.
\end{aligned}
$$

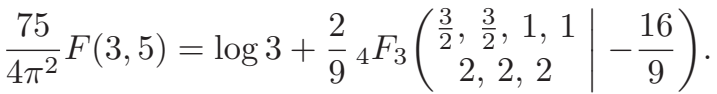

Все представления Бойда, отвечающие суммам $F(b, c)$ в таблице 3 , в настоящий момент строго доказаны, однако это и близко не покрывает полный список гипотез Бойда [34], [35]. Для “лакунарных" сумм $F(1,1), F(1,2), F(1,3)$ указанные представления получены Ф. Родригесом-Виллегасом [120]; А. Меллит [98] доказал формулу для $F(2,7)$, используя методы $K$-теории; представления для $F(1,5), F(2,3)$ и $F(3,5)$ доказаны в совместных работах автора с М. Роджерсом [124], [125].

Использованные в [124], [125] методы по сути достаточно элементарны. Интеграл

$$
F(a, b, c, d)=-\int_{0}^{1} \eta(a \tau) \eta(b \tau) \eta(c \tau) \eta(d \tau) \frac{\log q}{q} \mathrm{~d} q, \quad q=e^{2 \pi i \tau},
$$

представляется в виде

$$
F(a, b, c, d)=\int_{0}^{1} x(q) \log y(q) \mathrm{d} z(q),
$$

где $x(q), y(q)$ и $z(q)$ - модулярные функции относительно некоторой конгруэнцподгруппы группы $\mathrm{SL}_{2}(\mathbb{Z})$ (такой шаг можно выполнить различными способами). Далее, $x$ и $y$ записываются в виде алгебраических функций от $z$, т.е. $x(q)=X(z(q)), y(q)=Y(z(q))$. В результате такой подстановки $F(a, b, c, d)$ превращается в сложный интеграл от элементарных функций; например,

$$
F(2,3)=-\frac{\pi}{48} \int_{0}^{1 / 2} \frac{\sqrt{(1-2 p)(2-p)} \log \frac{p^{3}(2-p)}{1-2 p}}{\left(1-p^{2}\right) \sqrt{p}} \mathrm{~d} p .
$$

Последним шагом является сведение элементарного интеграла к мерам Малера, для чего применяются свойства гипергеометрических и эллиптических функций. Используя технику из [124], [125], можно получить неизвестные ранее гипергеометрические выражения для $F(1,1)$ и $F(1,3)$, а также вывести новое функциональное уравнение для меры Малера

$$
\mathrm{m}((1+x)(1+y)(x+y)-\alpha x y), \quad \alpha \in \mathbb{R} .
$$

Все это приводит к весьма нетривиальным тождествам для гипергеометрических функций, “чисто гипергеометрические” доказательства которых неизвестны. 
Имеется большое число результатов и гипотез, касающихся мер Малера от $n$ переменных при $n>2$, причиной возникновения которых по большей части являются $K$-теоретические соображения. Помимо (101), Смит [33] установил, что

$$
\mathrm{m}(1+x+y+z)=\frac{7 \zeta(3)}{2 \pi^{2}} .
$$

Далее, Родригес-Виллегас [56] обнаружил численно, что

$$
\begin{aligned}
\mathrm{m}(1+x+y+z+t) & \stackrel{?}{=}-\left(\frac{\sqrt{15}}{2 \pi}\right)^{5} \int_{0}^{1}\left(\eta^{3}(3 \tau) \eta^{3}(5 \tau)+\eta^{3}(\tau) \eta^{3}(15 \tau)\right) \frac{\log ^{3} q}{q} \mathrm{~d} q \\
\mathrm{~m}(1+x+y+z+t+w) & \stackrel{?}{=}\left(\frac{3}{\pi^{2}}\right)^{3} \int_{0}^{1} \eta^{2}(\tau) \eta^{2}(2 \tau) \eta^{2}(3 \tau) \eta^{2}(6 \tau) \frac{\log ^{4} q}{q} \mathrm{~d} q .
\end{aligned}
$$

Такие выражения рассматриваются в [122], а также в [31] в связи с короткими случайными блужданиями на плоскости.

\section{Список литературы}

[1] M. Aganagic, V. Bouchard, A. Klemm, "Topological strings and (almost) modular forms", Comm. Math. Phys., 277:3 (2008), 771-819.

[2] G. Almkvist, J. Guillera, "Ramanujan-like series for $1 / \pi^{2}$ and string theory", Preprint at arXiv: 1009.5202 [math.NT], 2010.

[3] G. Almkvist, C. van Enckevort, D. van Straten, W. Zudilin, "Tables of Calabi-Yau equations", Preprint at arXiv: math.AG/0507430, 2005-2010.

[4] G. Almkvist, D. van Straten, W. Zudilin, "Apéry limits of differential equations of order 4 and 5", Modular Forms and String Duality, Fields Inst. Commun. Ser., 54, Amer. Math. Soc. \& Fields Inst., Providence, RI, 2008, 105-123.

[5] G. Almkvist, D. van Straten, W. Zudilin, "Generalizations of Clausen's formula and algebraic transformations of Calabi-Yau differential equations", Proc. Edinburgh Math. Soc., 54 (2011) (to appear).

[6] G. Almkvist, W. Zudilin, "Differential equations, mirror maps and zeta values", Mirror Symmetry V, AMS/IP Studies in Adv. Math., 38, Amer. Math. Soc. \& International Press, Providence, RI, 2006, 481-515.

[7] Y. André, G-functions and geometry, Aspects Math., E13, Friedr. Vieweg \& Sohn, Braunschweig, 1989.

[8] G. E. Andrews, "Problems and prospects for basic hypergeometric functions", Theory and application of special functions, Proc. Advanced Sem., Math. Res. Center (Univ. Wisconsin, Madison, WI, 1975), Math. Res. Center, Univ. Wisconsin, 35, ed. R. A. Askey, Academic Press, New York, 1975, 191-224.

[9] G. E. Andrews, "The well-poised thread: An organized chronicle of some amazing summations and their implications", Ramanujan J., 1:1 (1997), 7-23.

[10] G. E. Andrews, R. Askey, R. Roy, Special functions, Encyclopedia Math. Appl., 71, Cambridge Univ. Press, Cambridge, 1999.

[11] G. E. Andrews, B. C. Berndt, Ramanujan's Lost Notebook. Part I, Springer-Verlag, New York, 2005; Part II, Springer-Verlag, New York, 2009.

[12] R. Apéry, "Irrationalité de $\zeta(2)$ et $\zeta(3)$ ", Journées arithmétiques de Luminy (Luminy, 1978), Astérisque, 61, 1979, 11-13.

[13] W. N. Bailey, Generalized hypergeometric series, Cambridge Tracts in Math., 32, Cambridge Univ. Press, Cambridge, 1935; 2nd reprinted ed.: Stechert-Hafner, New York-London, 1964. 
[14] A. Baker, J. Coates, "Fractional parts of powers of rationals", Math. Proc. Cambridge Philos. Soc., 77:2 (1975), 269-279.

[15] K. Ball, T. Rivoal, "Irrationalité d'une infinité de valeurs de la fonction zêta aux entiers impairs", Invent. Math., 146:1 (2001), 193-207.

[16] N.D. Baruah, B. C. Berndt, H.H. Chan, "Ramanujan's series for $1 / \pi$ : a survey", Amer. Math. Monthly, 116:7 (2009), 567-587.

[17] M. A. Bennett, "Fractional parts of powers of rational numbers", Math. Proc. Cambridge Philos. Soc., 114:2 (1993), 191-201.

[18] M. A. Bennett, "An ideal Waring problem with restricted summands", Acta Arith., 66:2 (1994), 125-132.

[19] B. C. Berndt, Ramanujan's notebooks. Part I, Springer-Verlag, New York, 1985; Part II, Springer-Verlag, New York, 1989; Part III, Springer-Verlag, New York, 1991; Part IV, Springer-Verlag, New York, 1994; Part V, Springer-Verlag, New York, 1998.

[20] M. J. Bertin, "Mesure de Mahler d'une famille de polynômes", J. Reine Angew. Math., 569 (2004), 175-188.

[21] F. Beukers, "A note on the irrationality of $\zeta(2)$ and $\zeta(3)$ ", Bull. London Math. Soc., 11:3 (1979), 268-272.

[22] F. Beukers, "Fractional parts of powers of rationals", Math. Proc. Cambridge Philos. Soc., 90:1 (1981), 13-20.

[23] F. Beukers, "Irrationality of $\pi^{2}$, periods of an elliptic curve and $\Gamma_{1}(5)$ ", Diophantine approximations and transcendental numbers (Luminy, 1982), Progr. Math., 31, Birkhäuser, Boston, MA, 1983, 47-66.

[24] F. Beukers, "Irrationality proofs using modular forms", Journées arithmétiques de Besançon" (Besançon, 1985), Astérisque, 147-148, 1987, 271-283.

[25] F. Beukers, "On Dwork's accessory parameter problem", Math. Z., 241:2 (2002), 425-444.

[26] F. Beukers, G. Heckman, "Monodromy for the hypergeometric function ${ }_{n} F_{n-1}$ ", Invent. Math., 95:2 (1989), 325-354.

[27] J.-P. Bézivin, "Indépendence linéaire des valeurs des solutions transcendantes de certaines équations fonctionnelles", Manuscripta Math., 61:1 (1988), 103-129.

[28] J. W. Bober, "Factorial ratios, hypergeometric series, and a family of step functions", J. London Math. Soc. (2), 79 (2009), 422-444.

[29] M. Bogner, Differentielle Galoisgruppen und Transformationstheorie für Calabi-Yau-Operatoren vierter Ordnung, Diploma-Thesis, Institut für Mathematik, Johannes Gutenberg-Universität, Mainz, 2008.

[30] J. M. Borwein, P. B. Borwein, Pi and the AGM; A study in analytic number theory and computational complexity, Wiley, New York, 1987.

[31] J. M. Borwein, A. Straub, J. Wan, W. Zudilin, "Densities of short uniform random walks", Preprint at arXiv: 1103.2995 [math.CA], 2011.

[32] P. Borwein, "On the irrationality of $\sum \frac{1}{q^{n}+r}$ ", J. Number Theory, 37 (1991), 253-259.

[33] D. W. Boyd, "Speculations concerning the range of Mahler's measure", Canad. Math. Bull., 24:4 (1981), 453-469.

[34] D. W. Boyd, "Mahler's measure and special values of $L$-functions", Experiment. Math., 7:1 (1998), 37-82.

[35] D. W. Boyd, "Mahler's measure and invariants of hyperbolic manifolds", Number theory for the millennium, I, A K Peters, Natick, MA, 2002, 127-143.

[36] F. Brunault, "Version explicite du théorème de Beilinson pour la courbe modulaire $X_{1}(N)$ ", C. R. Math. Acad. Sci. Paris, 343:8 (2006), 505-510.

[37] P. Bundschuh, K. Väänänen, "Arithmetical investigations of a certain infinite product", Compositio Math., 91 (1994), 175-199. 
[38] P. Bundschuh, K. Väänänen, "Linear independance of $q$-analogues of certain classical constants", Result. Math., 47 (2005), 33-44.

[39] P. Bundschuh, W. Zudilin, "Rational approximations to a $q$-analogue of $\pi$ and some other q-series", Diophantine Approximation, Dev. Math., 16, Springer-Verlag, Vienna, 2008, 123-139.

[40] P. Candelas, X. C. de la Ossa, P. S. Green, L. Parkes, "A pair of Calabi-Yau manifolds as an exactly soluble superconformal theory", Nuclear Phys. B, 359:1 (1991), 21-74.

[41] Y.-H. Chen, Y. Yang, N. Yui, "Monodromy of Picard-Fuchs differential equations for Calabi-Yau threefolds", with an appendix by C. Erdenberger, J. Reine Angew. Math., 616 (2008), 167-203.

[42] G. V. Chudnovsky, "On the method of Thue-Siegel", Ann. of Math. (2), 117:2 (1983), 325-382.

[43] D. V. Chudnovsky, G. V. Chudnovsky, "Approximations and complex multiplication according to Ramanujan", Ramanujan revisited (Urbana-Champaign, IL, 1987), Academic Press, Boston, MA, 1988, 375-472.

[44] J. Cullen, "Pi formula", Preprint (December 2010).

[45] E. Delaygue, "Critère pour l'intégralité des coefficients de Taylor des applications miroir", J. Reine Angew. Math. (to appear); Preprint at arXiv:0912.3776 [math.NT], 2009.

[46] F. Delmer, J.-M. Deshouillers, "The computation of $g(k)$ in Waring's problem", Math. Comp., 54 (1990), 885-893.

[47] C. Deninger, "Deligne periods of mixed motives, $K$-theory and the entropy of certain $\mathbb{Z}^{n}$-actions", J. Amer. Math. Soc., 10:2 (1997), 259-281.

[48] А. К. Дубицкас, "Оценка снизу величины $\left\|(3 / 2)^{k}\right\| ”$, УМH, 45:1 (1990), 153-154; англ. пер.: А. K. Dubitskas [A. Dubickas], "A lower bound on the value of $\left\|(3 / 2)^{k}\right\|$ ", Russian Math. Surveys, 45:4 (1990), 163-164.

[49] D. Duverney, "Irrationalité d'un $q$-analogue de $\zeta(2)$ ", C. R. Acad. Sci. Paris Sér. I Math., 321:10 (1995), 1287-1289.

[50] R. Dvornicich, C. Viola, "Some remarks on Beukers' integrals", Number theory, Vol. II (Budapest, 1987), Colloq. Math. Soc. János Bolyai, 51, North-Holland, Amsterdam, 1987, 637-657.

[51] S. B. Ekhad, D. Zeilberger, "A WZ proof of Ramanujan's formula for $\pi$ ", Geometry, Analysis, and Mechanics, ed. J. M. Rassias, World Sci. Publ., River Edge, NJ, 1994, 107-108.

[52] P. Erdös, "On arithmetical properties of Lambert series", J. Indiana Math. Soc. (N.S.), 12 (1948), 63-66.

[53] L. Euler, "Variae observationes circa series infinitas", Comm. Acad. Sci. Imp. Petropol., 9 (1737), 160-188; Reprint: Opera Omnia Ser. I, 14, Teubner, Berlin, 1925, 216-245.

[54] L. Euler, "Meditationes circa singulare serierum genus", Novi Comm. Acad. Sci. Petropol., 20 (1775), 140-186; Reprint: Opera Omnia Ser. I, 15, Teubner, Berlin, 1927, 217-267.

[55] S. R. Finch, Mathematical constants, Encyclopedia Math. Appl., 94, Cambridge University Press, Cambridge, 2003.

[56] S.R. Finch, "Modular forms on $\mathrm{SL}_{2}(\mathbb{Z})$ ", Preprint at http://algo.inria.fr/ csolve/frs.pdf, 2005.

[57] S. Fischler, "Irrationalité de valeurs de zêta [d'après Apéry, Rivoal, ...]", Astérisque, 294 (2004), 27-62.

[58] S. Fischler, W. Zudilin, "A refinement of Nesterenko's linear independence criterion with applications to zeta values", Math. Ann., 347:4 (2010), 739-763. 
[59] P. J. Forrester, M.L. Glasser, "Some new lattice sums including an exact result for the electrostatic potential within the $\mathrm{NaCl}$ lattice", J. Phys. A Math. Gen., 15 (1982), 911-914.

[60] J. Franel, "On a question of Laisant", L'intermédiaire des mathématiciens, 1, Gauthier-Villars, Paris, 1894, 45-47; "On a question of J. Franel", L'intermédiaire des mathématiciens, 2, Gauthier-Villars, Paris, 1895, 33-35.

[61] G. Gasper, M. Rahman, Basic hypergeometric series, 2nd ed., Encyclopedia Math. Appl., 96, Cambridge Univ. Press, Cambridge, 2004; русский пер. 1-го изд.: Г. Гаспер, М. Рахман, Базисные гипергеометрические ряды, Мир, М., 1993.

[62] А.О. Гельфонд, Исчисление конечных разностей, 3-е изд., Наука, М., 1967; англ. пер.: А. O. Gel'fond, Calculus of finite differences, Intern. Monographs Adv. Math. Phys., Hindustan Publishing Corp., Delhi, 1971.

[63] M. L. Glasser, "Evaluation of lattice sums. IV. A five-dimensional sum", J. Phys. A Math. Gen., 16 (1975), 1237-1238.

[64] R. L. Graham, D. E. Knuth, O. Patashnik, Concrete mathematics. A foundation for computer science, 2nd ed., Addison-Wesley, Reading, MA, 1994; русский пер.: Р. Грэхем, Д. Кнут, О. Паташник, Конкретная математика. Основание информатики, Мир, М., 1998.

[65] J. Guillera, "Some binomial series obtained by the WZ-method", Adv. in Appl. Math., 29:4 (2002), 599-603.

[66] J. Guillera, "About a new kind of Ramanujan-type series", Experiment. Math., 12:4 (2003), 507-510.

[67] J. Guillera, "Hypergeometric identities for 10 extended Ramanujan-type series", Ramanujan J., 15:2 (2008), 219-234.

[68] J. Guillera, "A new Ramanujan-like series for $1 / \pi^{2}$ ", Preprint at arXiv: 1003.1915 [math.NT], 2010.

[69] J. Guillera, "A matrix form of Ramanujan-type series fo $1 / \pi$ ", Gems in Experimental Mathematics, Contemp. Math., 517, eds. T. Amdeberhan, L. A. Medina, and V. H. Moll, Amer. Math. Soc., Providence, RI, 2010, 189-206.

[70] J. Guillera, "Mosaic supercongruences of Ramanujan type", Preprint at arXiv: 1007.2290 [math.NT], 2010.

[71] J. Guillera, W. Zudilin, "Divergent" Ramanujan-type supercongruences", Proc. Amer. Math. Soc. (to appear); Preprint at arXiv: 1004.4337 [math.NT], 2010.

[72] Л.А. Гутник, "Об иррациональности некоторых величин, содержащих $\zeta(3)$ ", УМH, 34:3 (1979), 190; Acta Arith., 42:3 (1983), 255-264; англ. пер.: L. A. Gutnik, "On the irrationality of some quantities containing $\zeta(3)$ ", Eleven papers translated from the Russian, Amer. Math. Soc. Transl. Ser. 2, 140, Amer. Math. Soc., Providence, RI, 1988, 45-55.

[73] A. J. Guttmann, "Lattice Green functions and Calabi-Yau differential equations", J. Phys. A Math. Theor., 42:23 (2009), 232001, 6 pp.

[74] L. Habsieger, "Explicit lower bounds for $\left\|(3 / 2)^{k}\right\| "$, Acta Arith., 106 (2003), 299-309.

[75] M. Hata, "Legendre type polynomials and irrationality measures", J. Reine Angew. Math., 407:1 (1990), 99-125.

[76] M. Hata, "A new irrationality measure for $\zeta(3)$ ", Acta Arith., 92:1 (2000), 47-57.

[77] Т. Г. Хессами Пилеруд, "О линейной независимости векторов с полилогарифмическими координатами”, Вестник МГУ. Сер. 1. Матем., мех., 1999, № 6, 54-56; англ. пер.: T. Hessami Pilehrood, "On the linear independence of vectors with polylogarithmic coordinates", Moscow Univ. Math. Bull., 54:6 (1999), 40-42.

[78] F. Jouhet, E. Mosaki, "Irrationalité aux entiers impairs positifs d'un $q$-analogue de la fonction zêta de Riemann", Intern. J. Number Theory, 6:5 (2010), 959-988. 
[79] C. Krattenthaler, T. Rivoal, Hypergéométrie et fonction zêta de Riemann, Mem. Amer. Math. Soc., 186, Amer. Math. Soc., Providence, RI, 2007, no. 875.

[80] C. Krattenthaler, T. Rivoal, "An identity of Andrews, multiple integrals, and very-well-poised hypergeometric series", Ramanujan J., 13 (2007), 203-219.

[81] C. Krattenthaler, T. Rivoal, "On a linear form for Catalan's constant", South East Asian J. Math. Sci., 6:2 (2008), 3-15.

[82] C. Krattenthaler, T. Rivoal, "On the integrality of the Taylor coefficients of mirror maps", Duke Math. J., 151:2 (2010), 175-218; "On the integrality of the Taylor coefficients of mirror maps. II", Commun. Number Theory Phys., 3:3 (2009), 555-591.

[83] C. Krattenthaler, T. Rivoal, "Multivariate $p$-adic formal congruences and integrality of Taylor coefficients of mirror maps", Théories galoisiennes et arithmétiques des équations différentielles, Séminaires et Congrès, eds. L. Di Vizio and T. Rivoal, Soc. Math. France, Paris (to appear); Preprint at arXiv: 0804.3049 [math.NT], 2008.

[84] C. Krattenthaler, T. Rivoal, W. Zudilin, "Séries hypergéométriques basiques, $q$-analogues des valeurs de la fonction zêta et formes modulaires", Inst. Jussieu Math. J., 5:1 (2006), 53-79.

[85] J. Kubina, M. Wunderlich, "Extending Waring's conjecture up to 471600000", Math. Comp., 55 (1990), 815-820.

[86] N. Kurokawa, H. Ochiai, "Mahler measures via crystalization", Comment. Math. Univ. St. Pauli, 54:2 (2005), 121-137.

[87] M. N. Lalín, M. D. Rogers, "Functional equations for Mahler measures of genus-one curves", Algebra Number Theory, 1:1 (2007), 87-117.

[88] D. H. Lehmer, "Factorization of certain cyclotomic functions", Ann. of Math. (2), 34:3 (1933), 461-479.

[89] B. H. Lian, S.-T. Yau, "Differential equations from mirror symmetry", Surveys in differential geometry: differential geometry inspired by string theory, Surv. Differ. Geom., 5, Intern. Press, Boston, MA, 1999, 510-526.

[90] F. Lindemann, "Über die Zahl $\pi$ ", Math. Ann., 20:2 (1882), 213-225.

[91] H. Maass, Siegel's modular forms and Dirichlet series, Lecture Notes in Math., 216, Springer-Verlag, Berlin-New York, 1971.

[92] K. Mahler, "On the fractional parts of powers of real numbers", Mathematika, 4:2 (1957), 122-124.

[93] K. Mahler, "An application of Jensen's formula to polynomials", Mathematika, 7:2 (1960), 98-100.

[94] K. Mahler, "On algebraic differential equations satisfied by automorphic functions", J. Austral. Math. Soc., 10 (1969), 445-450.

[95] V. Maillot, Géométrie d'Arakelov des variétés toriques et fibrés en droites intégrables, Mém. Soc. Math. France (N.S.), 80, Soc. Math. France, Paris, 2000.

[96] Y. Martin, K. Ono, "Eta-quotients and elliptic curves", Proc. Amer. Math Soc., 125:11 (1997), 3169-3176.

[97] T. Matalo-Aho, K. Väänänen, W. Zudilin, "New irrationality measures for q-logarithms", Math. Comp., 75:254 (2006), 879-889.

[98] A. Mellit, "Elliptic dilogarithms and parallel lines", Preprint, 2009.

[99] H. Movasati, "Eisenstein type series for Calabi-Yau varieties", Preprint at arXiv: 1007.4181 [math.AG], 2010.

[100] Ю.В.Нестеренко, "О линейной независимости чисел”, Вестник МГУ. Сер. 1. Maтем., мех., 1985, № 1, 46-54; англ. пер.: Yu. V. Nesterenko, "Linear independence of numbers", Moscow Univ. Math. Bull., 40:1 (1985), 69-74.

[101] Ю. В. Нестеренко, "Некоторые замечания о Ц(3)", Матем. заметки, 59:6 (1996), 865-880; англ. пер.: Yu. V. Nesterenko, "Some remarks on $\zeta(3)$ ", Math. Notes, 59:6 (1996), 625-636. 
[102] Ю.В. Нестеренко, "Модулярные функции и вопросы трансцендентности,", Maтем. сб., 187:9 (1996), 65-96; англ. пер.: Yu. V. Nesterenko, "Modular functions and transcendence questions", Sb. Math., 187:9 (1996), 1319-1348.

[103] Е. М. Никишин, "Об иррациональности значений функций $F(x, s)$ ", Maтем. сб., 109:3 (1979), 410-417; англ. пер.: E. M. Nikishin, "Irrationality of values of functions $F(x, s)$ ", Math. USSR Sb., 37:3 (1979), 381-388.

[104] K. Nishioka, "A conjecture of Mahler on automorphic functions", Arch. Math., 53:1 (1989), 46-51.

[105] M. Petkovšek, H. S. Wilf, D. Zeilberger, $A=B$, A K Peters, Wellesley, 1996.

[106] A. van der Poorten, "A proof that Euler missed... Apéry's proof of the irrationality of $\zeta(3) "$, Math. Intelligencer, 1:4 (1978/79), 195-203.

[107] K. Postelmans, W. Van Assche, "Irrationality of $\zeta_{q}(1)$ and $\zeta_{q}(2)$ ", J. Number Theory, 126 (2007), 119-154.

[108] M. Prévost, "A new proof of the irrationality of $\zeta(3)$ using Padé approximants", $J$. Comput. Appl. Math., 67 (1996), 219-235.

[109] Ю.А. Пупырев, "О линейной и алгебраической независимости $q$-дзета-значений", Матем. заметки, 78:4 (2005), 608-613; англ. пер.: Yu. A. Pupyrev, "Linear and algebraic independence of q-zeta values", Math. Notes, 78:4 (2005), 563-568.

[110] Ю.А. Пупырев, “Эффективизация нижней оценки для $\left\|(4 / 3)^{k}\right\|$ ", Матем. заметки, 85:6 (2009), 927-935; англ. пер.: Yu. A. Pupyrev, "Effectivization of a lower bound for $\left\|(4 / 3)^{k}\right\| "$, Math. Notes, 85:6 (2009), 877-885.

[111] S. Ramanujan, "Modular equations and approximations to $\pi$ ", Quart. J. Math. Oxford Ser. (2), 45 (1914), 350-372; Reprinted: Collected papers of Srinivasa Ramanujan, eds. G.H. Hardy, P. V. Sechu Aiyar, and B. M. Wilson, Cambridge University Press \& Chelsea Publ., New York, 1962, 23-39.

[112] G. Rhin, C. Viola, "On a permutation group related to $\zeta(2)$ ", Acta Arith., 77:1 (1996), 23-56.

[113] G. Rhin, C. Viola, "The group structure for $\zeta(3)$ ", Acta Arith., 97:3 (2001), 269-293.

[114] O. Richter, "On transformation laws for theta functions", Rocky Mountain J. Math., 34:4 (2004), 1473-1481.

[115] Б. Риман, "О числе простых чисел, не превышающих данной величины", Сочинения, ОГИЗ, М., 1948, 216-224; пер. с нем.: В. Riemann, "Ueber die Anzahl der Primzahlen unter einer gegebenen Grösse", Monatsberichte der Berliner Akademie, 1859; Reprint: Gesammelte Werke, Teubner, Leipzig, 1892.

[116] T. Rivoal, "La fonction zêta de Riemann prend une infinité de valeurs irrationnelles aux entiers impairs", C. R. Acad. Sci. Paris Sér. I Math., 331:4 (2000), 267-270.

[117] T. Rivoal, Propriétés diophantiennes des valeurs de la fonction zêta de Riemann aux entiers impairs, Thèse de Doctorat, Univ. de Caen, Caen, 2001.

[118] T. Rivoal, "Irrationalité d'au moins un des neuf nombres $\zeta(5), \zeta(7), \ldots, \zeta(21)$ ", Acta Arith., 103 (2002), 157-167.

[119] T. Rivoal, W. Zudilin, "Diophantine properties of numbers related to Catalan's constant", Math. Ann., 326:4 (2003), 705-721.

[120] F. Rodríguez-Villegas, "Modular Mahler measures I", Topics in number theory (University Park, PA, 1997), Math. Appl., 467, Kluwer Acad. Publ., Dordrecht, 1999, $17-48$.

[121] F. Rodríguez-Villegas, "Identities between Mahler measures", Number theory for the millennium, III, A K Peters, Natick, MA, 2002, 223-229.

[122] F. Rodríguez-Villegas, R. Toledano, J.D. Vaaler, "Estimates for Mahler's measure of a linear form", Proc. Edinburgh Math. Soc., 47:2 (2004), 473-494.

[123] M.D. Rogers, "Hypergeometric formulas for lattice sums and Mahler measures", Intern. Math. Res. Not. (to appear). 
[124] M.D. Rogers, W. Zudilin, "From $L$-series of elliptic curves to Mahler measures", Preprint at arXiv: 1012.3036 [math.NT], 2010.

[125] M. D. Rogers, W. Zudilin, "On the Mahler measure of $1+X+1 / X+Y+1 / Y$ ", Preprint at arXiv: 1102.1153 [math.NT], 2011.

[126] Е. А. Рухадзе, "Оценка снизу приближения $\ln 2$ рациональными числами”, Becmник МГУ. Сер. 1. Матем., мех., 1987, №6, 25-29; англ. пер.: E. A. Rukhadze, "Lower estimate for rational approximations of $\ln 2 "$, Moscow Univ. Math. Bull., 42:6 (1987), 30-35.

[127] A. L. Schmidt, "Generalized q-Legendre polynomials", J. Comput. Appl. Math., 49:1-3 (1993), 243-249.

[128] A. L. Schmidt, "Legendre transforms and Apéry's sequences", J. Austral. Math. Soc. Ser. A, 58:3 (1995), 358-375.

[129] А.Б. Шидловский, Трансцендентные числа, Наука, М., 1987; англ. пер.: A. B. Shidlovskii, Transcendental numbers, de Gruyter Stud. Math., 12, Walter de Gruyter \& Co., Berlin, 1989.

[130] L. J. Slater, Generalized hypergeometric functions, Cambridge Univ. Press, Cambridge, 1966.

[131] C. Smet, W. Van Assche, "Irrationality proof of a $q$-extension of $\zeta(2)$ using little q-Jacobi polynomials", Acta Arith., 138:2 (2009), 165-178.

[132] В.Н. Сорокин, "Аппроксимации Эрмита-Паде для систем Никишина и иррациональность $\zeta(3)$ ”, УМH, 49:2 (1994), 167-168; англ. пер.: V. N. Sorokin, "Hermite-Padé approximations for Nikishin systems and the irrationality of $\zeta(3)$ ", Russian Math. Surveys, 49:2 (1994), 176-177.

[133] В. Н. Сорокин, "О мере трансцендентности числа $\pi^{2}$ ", Матем. сб., 187:12 (1996), 87-120; англ. пер.: V. N. Sorokin, "A transcendence measure for $\pi^{2}$ ", Sb. Math., 187:12 (1996), 1819-1852.

[134] В.Н. Сорокин, "Теорема Апери", Вестник МГУ. Сер. 1. Матем., мех., 1998, № 3, 48-53; англ. пер.: V. N. Sorokin, "On Apéry’s theorem", Moscow Univ. Math. Bull., 53:3 (1998), 48-52.

[135] В.Н. Сорокин, "Циклические графы и теорема Апери", УМH, 57:3 (2002), 99-134; англ. пер.: V. N. Sorokin, "Cyclic graphs and Apéry's theorem", Russian Math. Surveys, 57:3 (2002), 535-571.

[136] V. Strehl, "Binomial identities - combinatorial and algorithmic aspects,", Discrete Math., 136:1-3 (1994), 309-346.

[137] Z.-W. Sun, "Supercongruences and Euler sums", Preprint at arXiv: 1001.4453 [math.NT], 2010.

[138] Y. Tachiya, "Irrationality of certain Lambert series", Tokyo J. Math., 27:1 (2004), 75-85.

[139] W. Van Assche, "Little $q$-Legendre polynomials and irrationality of certain Lambert series", Ramanujan J., 5 (2001), 295-310.

[140] О.Н. Василенко, "Некоторые формулы для значения дзета-функции Римана в целых точках", Теория чисел и ее приложения (Ташкент, 26-28 сентября 1990 г.), Тезисы докладов Республиканской научно-теоретической конференции, Ташкентский гос. пед. институт, Ташкент, 1990, 27.

[141] Д. В. Васильев, "Некоторые формулы для дзета-функции в целых точках", Вестник МГУ. Сер. 1. Матем., мех., 1996, № 1, 81-84; англ. пер.: D. V. Vasil'ev, "Some formulas for the Riemann zeta function at integer points", Moscow Univ. Math. Bull., 51:1 (1996), 41-43.

[142] D. V. Vasilyev, On small linear forms for the values of the Riemann zeta-function at odd points, Preprint № 1 (558), Nat. Acad. Sci. Belarus, Institute Math., Minsk, 2001. 
[143] R. C. Vaughan, The Hardy-Littlewood method, 2nd ed., Cambridge Tracts in Math., 125, Cambridge Univ. Press, Cambridge, 1997; русский пер. 1-го изд.: Р. Вон, Метод Харди-Литтлвуда, Мир, М., 1985.

[144] C. Viola, "Birational transformations and values of the Riemann zeta-function", $J$. Théor. Nombres Bordeaux, 15:2 (2003), 561-592.

[145] С. М. Воронин, А. А. Карацуба, Дзета-функиия Римана, Физматлит, М., 1994; англ. пер.: А. A. Karatsuba, S. M. Voronin, The Riemann zeta-function, de Gruyter Exp. Math., 5, Walter de Gruyter \& Co., Berlin, 1992.

[146] S. O. Warnaar, W. Zudilin, "A q-rious positivity", Aequat. Math., 81:1-2 (2011), 177-183.

[147] F. J. W. Whipple, "A group of generalized hypergeometric series: relations between 120 allied series of the type $F[a, b, c ; d, e]$ ", Proc. London Math. Soc. (2), 23 (1925), 104-114.

[148] F. J.W. Whipple, "On well-poised series, generalized hypergeometric series having parameters in pairs, each pair with the same sum", Proc. London Math. Soc. (2), 24 (1926), 247-263.

[149] H.S. Wilf, D. Zeilberger, "An algorithmic proof theory for hypergeometric (ordinary and "q") multisum/integral identities", Invent. Math., 108:3 (1992), 575-633.

[150] Y. Yang, "Apéry limits and special values of $L$-functions", J. Math. Anal. Appl., 343:1 (2008), 492-513.

[151] Y. Yang, W. Zudilin, "An $\mathrm{Sp}_{4}$ modularity of Picard-Fuchs differential equations for Calabi-Yau threefolds", with an appendix by V. Pasol, Gems in Experimental Mathematics, Contemp. Math., 517, eds. T. Amdeberhan, L. A. Medina, and V. H. Moll, Amer. Math. Soc., Providence, RI, 2010, 381-413.

[152] M. Yoshida, Fuchsian differential equations. With special emphasis on the Gauss-Schwarz theory, Aspects Math., E11, Friedr. Vieweg \& Sohn, Braunschweig, 1987.

[153] D. Zagier, "The non-holomorphic embedding of $\mathfrak{H}$ into $\mathfrak{H}_{2}$ ", Unpublished note, 2008.

[154] D. Zagier, "Integral solutions of Apéry-like recurrence equations", Groups and Symmetries: From Neolithic Scots to John McKay, CRM Proc. Lecture Notes, 47, Amer. Math. Soc., Providence, RI, 2009, 349-366.

[155] D. Zeilberger, "Computerized deconstruction", Adv. Appl. Math., 31 (2003), $532-543$.

[156] С.А. Злобин, "Интегралы, представляемые в виде линейных форм от обобщенных полилогарифмов", Матем. заметки, 71:5 (2002), 782-787; англ. пер.: S. A. Zlobin, "Integrals expressible as linear forms in generalized polylogarithms", Math. Notes, 71:5 (2002), 711-716.

[157] С.А. Злобин, "О некоторых интегральных тождествах", УМН, 57:3 (2002), 153-154; англ. пер.: S. A. Zlobin, "On some integral identities", Russian Math. Surveys, 57:3 (2002), 617-618.

[158] С. А. Злобин, "Интегралы Рина", Матем. заметки, 81:2 (2007), 226-239; англ. пер.: S. A. Zlobin, "Rhin integrals", Math. Notes, 81:2 (2007), 201-212.

[159] I. J. Zucker, "Madelung constants and lattice sums for hexagonal crystals", J. Phys. A Math. Gen., 24:4 (1991), 873-879.

[160] В. В. Зудилин, "Разностные уравнения и мера иррациональности чисел", Аналитическая теория чисел и приложения, Труды МИАН, 218, 1997, 165-178; англ. пер.: W. Zudilin, "Difference equations and the irrationality measure of numbers", Proc. Steklov Inst. Math., 218 (1997), 160-174.

[161] W. Zudilin, "Number theory casting a look at the mirror", Preprint at arXiv: math. NT/0008237, 2000. 
[162] В. В. Зудилин, "Одно из чисел $\zeta(5), \zeta(7), \zeta(9), \zeta(11)$ иррационально”, $У М H, ~ 56: 4$ (2001), 149-150; англ. пер.: W. Zudilin, "One of the numbers $\zeta(5), \zeta(7), \zeta(9), \zeta(11)$ is irrational", Russian Math. Surveys, 56:4 (2001), 774-776.

[163] В.В. Зудилин, "Об иррациональности значений дзета-функции Римана”, Изв. РАН. Серия матем., 66:3 (2002), 49-102; англ. пер.: W. Zudilin, "Irrationality of values of the Riemann zeta function", Izv. Math., 66:3 (2002), 489-542.

[164] W. Zudilin, "Remarks on irrationality of q-harmonic series", Manuscripta Math., 107:4 (2002), 463-477.

[165] В. В. Зудилин, "О мере иррациональности $q$-аналога $\zeta(2) "$, Матем. сб., 193:8 (2002), 49-70; англ. пер.: W. Zudilin, "On the irrationality measure for a $q$-analogue of $\zeta(2)$ ", Sb. Math., 193:8 (2002), 1151-1172.

[166] В. В. Зудилин, "Совершенно уравновешенные гипергеометрические ряды и кратные интегралы", УМH, 57:4 (2002), 177-178; англ. пер.: W. Zudilin, "Very well-poised hypergeometric series and multiple integrals", Russian Math. Surveys, 57:4 (2002), 824-826.

[167] В.В. Зудилин, "О рекурсии третьего порядка типа Апери для $\zeta(5)$ ", Матем. заметки, 72:5 (2002), 796-800; англ. пер.: W. Zudilin, "A third-order Apéry-like recursion for $\zeta(5)$ ", Math. Notes, 72:5 (2002), 733-737.

[168] В. В. Зудилин, "О диофантовых задачах для $q$-дзета-значений”, Матем. заметки, 72:6 (2002), 936-940; англ. пер.: W. Zudilin, "Diophantine problems for q-zeta values", Math. Notes, 72:6 (2002), 858-862.

[169] W. Zudilin, "The hypergeometric equation and Ramanujan functions", Ramanujan J., $7: 4(2003), 435-447$.

[170] В. В. Зудилин, "О функциональной трансцендентности $q$-дзета-значений”, $M a-$ тем. заметки, 73:4 (2003), 629-630; англ. пер.: W. Zudilin, "On the functional transcendence of $q$-zeta values", Math. Notes, 73:4 (2003), 588-589.

[171] W. Zudilin, "Well-poised hypergeometric service for diophantine problems of zeta values", J. Théor. Nombres Bordeaux, 15:2 (2003), 593-626.

[172] W. Zudilin, "Heine's basic transform and a permutation group for $q$-harmonic series", Acta Arith., 111:2 (2004), 153-164.

[173] W. Zudilin, "Arithmetic of linear forms involving odd zeta values", J. Théor. Nombres Bordeaux, 16:1 (2004), 251-291.

[174] W. Zudilin, "Well-poised hypergeometric transformations of Euler-type multiple integrals", J. London Math. Soc., 70:1 (2004), 215-230.

[175] В. В. Зудилин, "О биномиальных суммах, связанных с рациональными приближениями к Ц(4)", Матем. заметки, 75:4 (2004), 637-640; англ. пер.: W. Zudilin, "Binomial sums related to rational approximations to $\zeta(4)$ ", Math. Notes, 75:4 (2004), 594-597.

[176] W. Zudilin, "On a combinatorial problem of Asmus Schmidt", Electron. J. Combin., 11:1 (2004), \#R22, 8 pages.

[177] В. В. Зудилин, "Об обратном преобразовании Лежандра одного семейства последовательностей", Матем. заметки, 76:2 (2004), 300-303; англ. пер.: W. Zudilin, "The inverse Legendre transform of a certain family of sequences", Math. Notes, $\mathbf{7 6 : 2}$ (2004), 276-279.

[178] W. Zudilin, "Approximations to $q$-logarithms and $q$-dilogarithms, with applications to q-zeta values", Труды по теории чисел, Зап. научн. сем. ПОМИ, 322, 2005, 107-124; Reprinted: J. Math. Sci. (N. Y.), 137:2 (2006), 4673-4683.

[179] W. Zudilin, "A new lower bound for $\left\|(3 / 2)^{k}\right\|$ ", J. Théor. Nombres Bordeaux, 19:1 (2007), 313-325.

[180] W. Zudilin, "Approximations to -, di- and tri-logarithms", J. Comput. Appl. Math., 202:2 (2007), 450-459. 
[181] W. Zudilin, "Ramanujan-type formulae for $1 / \pi$ : A second wind?", Modular Forms and String Duality, Fields Inst. Commun. Ser., 54, Amer. Math. Soc. \& Fields Inst., Providence, RI, 2008, 179-188.

[182] W. Zudilin, "Apéry's theorem. Thirty years after", Intern. J. Math. Computer Sci., 4:1 (2009), 9-19.

[183] W. Zudilin, "Ramanujan-type supercongruences", J. Number Theory, 129:8 (2009), $1848-1857$.

В. В. Зудилин (W. Zudilin)

School of Mathematical and Physical Sciences, University of Newcastle, Callaghan, Australia

E-mail: wadim.zudilin@newcastle.edu.au
Поступила в редакцию 18.02.2011 